\title{
Hölder-Sobolev regularity of the solution to the stochastic wave equation in dimension 3
}

by

\author{
Robert C. Dalang ${ }^{(*)}$ \\ Institut de Mathématiques \\ Ecole Polytechnique Fédérale \\ Station 8 \\ CH-1015 Lausanne, Switzerland \\ e-mail: robert.dalang@epfl.ch
}

\author{
and \\ MARTA SANZ-SolÉ ${ }^{(* *)}$
Facultat de Matemàtiques
Universitat de Barcelona
Gran Via 585
E-08007 Barcelona, Spain
e-mail: marta.sanz@ub.edu
}

Abstract: We study the sample path regularity of the solution of a stochastic wave equation in spatial dimension $d=3$. The driving noise is white in time and with a spatially homogeneous covariance defined as a product of a Riesz kernel and a smooth function. We prove that at any fixed time, a.s. the sample paths in the spatial variable belong to certain fractional Sobolev spaces. In addition, for any fixed $x \in \mathbb{R}^{3}$, the sample paths in time are Hölder continuous functions. Hence, we obtain joint Hölder continuity in the time and space variables. Our results rely on a detailed analysis of properties of the stochastic integral used in the rigourous formulation of the spde, as introduced by Dalang and Mueller (2003). Sharp results on one and two dimensional space and time increments of generalized Riesz potentials are a crucial ingredient in the analysis of the problem. For spatial covariances given by Riesz kernels, we show that the Hölder exponents that we obtain are optimal.

Key words and phrases. Stochastic partial differential equations, sample path regularity, spatially homogeneous random noise, wave equation.

MSC 2000 Subject Classifications. Primary 60H15; Secondary 60J45, 35R60, 35L05.

(*) Partially supported by the Swiss National Foundation for Scientific Research.

(**) Partially supported by the grant BFM2003-01345 from Dirección General de Investigación, Ministerio de Ciencia y Tecnología, Spain. 


\section{Introduction}

This paper studies the stochastic wave equation in spatial dimension $d=3$

$$
\begin{gathered}
\left(\frac{\partial^{2}}{\partial t^{2}}-\Delta\right) u(t, x)=\sigma(u(t, x)) \dot{F}(t, x)+b(u(t, x)), \\
u(0, x)=v_{0}(x), \quad \frac{\partial}{\partial t} u(0, x)=\tilde{v}_{0}(x),
\end{gathered}
$$

where $t \in] 0, T]$ for some fixed $T>0, x \in \mathbb{R}^{3}$ and $\Delta$ denotes the Laplacian on $\mathbb{R}^{3}$. The coefficients $\sigma$ and $b$ are Lipschitz continuous functions, the process $\dot{F}$ is the formal derivative of a Gaussian random field, white in time and correlated in space. More precisely, for any $d \geq 1$, let $\mathcal{D}\left(\mathbb{R}^{d+1}\right)$ be the space of Schwartz test functions (see [25]) and let $\Gamma$ be a non-negative and nonnegative definite tempered measure on $\mathbb{R}^{d}$. Then, on some probability space, there exists a Gaussian process $F=\left(F(\varphi), \varphi \in \mathcal{D}\left(\mathbb{R}^{d+1}\right)\right)$ with mean zero and covariance functional given by

$$
E(F(\varphi) F(\psi))=\int_{\mathbb{R}_{+}} d s \int_{\mathbb{R}^{d}} \Gamma(d x)(\varphi(s) * \tilde{\psi}(s))(x),
$$

where $\tilde{\psi}(s)(x)=\psi(s)(-x)$.

We are interested in solutions which are random fields, that is, real-valued processes $\left(u(t, x),(t, x) \in[0, T] \times \mathbb{R}^{3}\right)$, that are well defined for every fixed $(t, x) \in[0, T] \times \mathbb{R}^{3}$. We want to study their sample path regularity, both in time and space, and check the optimality of the results.

There are different possible approaches to giving a rigourous formulation of the Cauchy problem (1.1). However, in all of them the fundamental solution associated to the wave operator $\mathcal{L}=\frac{\partial^{2}}{\partial t^{2}}-\Delta$ naturally plays an important role. Since its singularity increases with the spatial dimension $d$, the difficulties in studying regularity of the solutions of the stochastic wave equation increase accordingly. Moreover, keeping the requirement of obtaining random field solutions amounts to adjusting the roughness of the noise to the degeneracy of the differential operator which defines the equation. It is only for $d=1$ that it is possible to take a space-time white noise as random input to (1.1), while in higher dimensions a non-degenerate spatial correlation is necessary $[6,12]$.

For $d=1,2$, the stochastic wave equation driven by space-time white noise, and noise that is white in time but spatially correlated, respectively, is now quite well understood. We refer the reader to [3], [4], [5], [12], [14], [16], [17], [19], [20], for a sample of articles on the subject. 
For $d=3$, the fundamental solution of the wave equation is the measure defined by

$$
G(t)=\frac{1}{4 \pi t} \sigma_{t}
$$

for any $t>0$, where $\sigma_{t}$ denotes the uniform surface measure (with total mass $\left.4 \pi t^{2}\right)$ on the sphere of radius $t \in[0, T]$. Hence, in the mild formulation of equation (1.1), Walsh's classical stochastic integration theory developed in [32] does not apply. In fact, this question motivated two different extensions of Walsh's integral, given in [6] and [7], respectively.

The stochastic integral of [6], written

$$
\int_{0}^{t} G(t-s, y) Z(s, y) M(d s, d y)
$$

requires a non-negative distribution $G$, a second-order stationary process $Z$ in the integrand, and the integrability condition

$$
\int_{0}^{T} d s \int_{\mathbb{R}^{d}} \mu(d \xi)|\mathcal{F} G(t)(\xi)|^{2}<\infty
$$

where $\mu=\mathcal{F}^{-1} \Gamma$, among other technical properties. As is shown in Section 5 of [6], with this integral one can obtain existence and uniqueness of a random field solution to (1.1), interpreted in the mild form (4.2), in the case where the initial conditions vanish. In this framework, results on the regularity of the law of the solution to the stochastic wave equation have been proved in [21] and [22] (see also [27]).

In [7], a new extension of Walsh's stochastic integral based on a functional approach is introduced. Neither the positivity of $G$ nor the stationarity of $Z$ are required (see [7], Theorem 6). With this integral, the authors give a precise meaning to the problem (1.1) with non vanishing initial conditions and coefficient $b \equiv 0$ and obtain existence and uniqueness of a solution $(u(t), t \in[0, T])$ which is an $L^{2}\left(\mathbb{R}^{3}\right)$-valued stochastic process (Theorem 9 in [7]). This is the choice of stochastic integral that we will use in this paper to study the stochastic wave equation (1.1).

We consider the particular case of a covariance measure that is absolutely continuous with respect to Lebesgue measure, with density given by

$$
f(x)=\varphi(x) k_{\beta}(x)
$$

where $\varphi$ is a smooth positive function and $k_{\beta}$ denotes the Riesz kernel $k_{\beta}(x)=$ $|x|^{-\beta}$, with $\left.\beta \in\right] 0,2[$ (see Assumption 2.4). Riesz kernels are a natural class of correlation functions and are already present in previous work on the stochastic heat and wave equations, for instance in [5], [6], [12], [16]. They 
provide examples where condition (1.4) is satisfied: for these covariances, (1.4) is equivalent to the condition $0<\beta<2$ (see Example 2.5).

Related questions for an equation that is second order in time but with fractional Laplacian in any spatial dimension $d$ and general covariance measure $\Gamma$ have been considered in [8], in the setting of an $L^{2}$-theory (see [24]). The results there are shown to be optimal in time. We adopt here a similar strategy, but we work in an $L^{q}$-framework (see [13]), for any $q \geq 2$. Indeed, the particular structure of the wave equation in dimension $d=3$ makes it possible to go beyond the Hilbert space setting and to obtain sharp results, both in time and space.

The main result of the paper is Theorem 4.11, stating joint Höldercontinuity in $(t, x)$ of the solution to (1.1), together with the analysis of the optimality of the exponents studied in Section 5. The optimal Hölder exponent is the same for the time and space variables: this is an intrinsic property of the d'Alembert operator. Moreover, this result shows how the driving noise $\dot{F}$ contributes to the roughness of the sample paths, since it expresses the optimal Hölder exponent in terms of the parameters $\beta$ and $\delta$ appearing in Assumption 2.4 on $\dot{F}$ (see Section 2.2).

Notice that for the stochastic heat equation with Lipschitz coefficients in any spatial dimension $d \geq 1$, joint Hölder-continuity in $(t, x)$ of the sample paths of the solution has been established in [26]. Unlike the stochastic wave equation, the Hölder exponent in the time variable is half that for the spatial variable. This is also an intrinsic property of the heat operator. However, it turns out that effect of the driving noise $\dot{F}$ on the regularity in the spatial variable is the same for both equations (see Theorem 4.11 and Remark 4.8). Similar problems for non-Lipschitz coefficients have been recently tackled in [18].

We should point out that despite the similarities just mentioned, establishing regularity results for the solution of the stochastic wave equation requires fundamentally different methods than those for the stochastic heat equation. Indeed, taking for simplicity $b \equiv 0$ and vanishing initial conditions, equation (1.1), written in integral form, becomes

$$
u(t, x)=\int_{0}^{t} \int_{\mathbb{R}^{d}} G(t-s, x-v) Z(s, v) \dot{F}(d s, d v),
$$

where $Z(s, v)=\sigma(u(s, v))$. A spatial increment of the solution is

$$
u(t, x)-u(t, y)=\int_{0}^{t} \int_{\mathbb{R}^{d}}(G(t-s, x-v)-G(t-s, y-v)) Z(s, v) \dot{F}(d s, d v) .
$$

When the fundamental solution $G$ is smooth, as in the case of the heat 
equation, one uses Burholder's inequality to see that

$$
\begin{aligned}
& E\left(|u(t, x)-u(t, y)|^{p}\right) \\
& \leq C E\left(\int_{0}^{t} d s \int_{\mathbb{R}^{d}} d u \int_{\mathbb{R}^{d}} d v(G(t-s, x-u)-G(t-s, y-u)) Z(s, u)\right. \\
& \quad \times f(u-v) Z(s, v)(G(t-s, x-v)-G(t-s, y-v))^{p / 2} .
\end{aligned}
$$

Then, the smoothness of $G$, together with integrability of $Z$, leads to regularity of $u(t, \cdot)$. For the wave equation, $G(t)$ is singular with respect to Lebesgue measure (see (1.3)), so this kind of approach is not feasible.

A different idea is to pass the increments on $G$ in (1.6) onto the factor $Z(s, u) f(u-v) Z(s, v)$, using a change of variables; the right-hand side of (1.6) becomes the sum of

$-\int_{0}^{t} d s \int_{\mathbb{R}^{3}} G(s, d u) \int_{\mathbb{R}^{3}} G(s, d v) D^{2} f(v-u, x-y) E(Z(t-s, x-u) Z(t-s, x-v))$,

where $D^{2} f(u, x)=f(u+x)-2 f(u)+f(u-x)$, and of three other terms of similar form (see the proof of Lemma 3.2 for details). Focussing on the term (1.7), one checks that in the case where $f(x)=|x|^{-\beta}$ is a Riesz kernel,

$$
\left|D^{2} f(u, x)\right| \leq c\left|f^{\prime \prime}(u)\right||x|^{2} \leq c|u|^{-(\beta+2)}|x|^{2},
$$

where $f^{\prime \prime}$ denotes the second order diferential of $f$. This would lead to the following bound for (1.7):

$$
|x-y|^{2} \int_{0}^{t} d s \int_{\mathbb{R}^{3}} G(s, d u) \int_{\mathbb{R}^{3}} G(s, d v)|v-u|^{-(\beta+2)} .
$$

The factor $|x-y|^{2}$ looks too good to be true, and it is! Indeed, the triple integral is equal to the left-hand side of (1.4), and we have already pointed out that this is finite if and only if the exponent $\beta+2$ is less than 2 . However, this is not the case since $\beta \in] 0,2[$ !

Even though the bound (1.9) equals $+\infty$, this approach contains the premises of our argument. Indeed, instead of differentiating $f$ twice as in (1.8), we shall estimate $D^{2} f$ by using a fractional derivative of order $\gamma$, where $\gamma<2-\beta$. It turns out that for $f(u)=|u|^{-\beta}$, the fractional derivative $f^{(\gamma)}(u) \simeq|u|^{-(\beta+\gamma)}$. This leads to the following bound for (1.7):

$$
|x-y|^{\gamma} \int_{0}^{t} d s \int_{\mathbb{R}^{3}} G(s, d u) \int_{\mathbb{R}^{3}} G(s, d v)|v-u|^{-(\beta+\gamma)} .
$$

The triple integral is now finite since $\beta+\gamma<2$ and this gives the correct order of regularity for $u(t, \cdot)$. The precise properties of Riesz kernels and rigorous 
use of their fractional derivatives (or rather, their fractional Laplacians) are given in Lemma 2.6 (with $a$ there replaced by $3-\beta-\gamma, b$ replaced by $\gamma$, and $d=3)$.

In short, there are mainly three ideas which have been central to obtaining the results of this paper. First, the smoothing in space of the fundamental solution by means of a regularisation procedure based on time-scaled approximations of the Dirac delta-function (see (2.1)), and the study of the corresponding smoothed equation, to which we can successfully apply standard techniques of stochastic calculus. Secondly, at the level of the smoothed equation, increments of stochastic integrals, whether in space or in time, initially expressed in terms of increments of the fundamental solution, can be reexpressed in terms of increments of the covariance density of the noise. Using the semigroup property of Riesz potentials (see for instance [30]), we implement the ideas concerning fractional derivatives described above (see Lemma 2.6). With these results, we are able to obtain bounds on one and two dimensional increments, in space and in time, of certain generalized Riesz potentials of a smoothed version of the fundamental solution of the wave equation. The sharp character of these estimates leads to the optimality of our results.

The paper is organized as follows. In Section 2, we define the smoothing $G_{n}$ of the fundamental solution $G$ and prove some of their basic properties. Then we describe precisely the type of stochastic noise we are considering in the paper and prove the above mentioned fractional derivative properties.

In Section 3, we study the path properties of the indefinite stochastic integral introduced in [7]. Briefly stated, we prove that if the sample paths of the stochastic integrand belong to some fractional Sobolev space with a fixed order of differentiability, then the stochastic integral inherits the same property with a related order of differentiability (Theorem 3.1). This fact, together with Sobolev's embeddings and $L^{p}$-estimates of increments in time of the integral (Theorem 3.5), complete the analysis.

Section 4 is devoted to the study of equation (1.1) itself. The idea is to transfer the properties of the stochastic integral obtained in Section 3 to the solution of the equation. First, in Section 4.1, we give a more general version of existence and uniqueness of a solution and its properties than in [7], allowing non vanishing initial conditions and an additive non-linearity $b$. We also show how the $L^{q}$-moments of the solution depend on properties of the initial conditions (see Theorem 4.1).

Next, in Section 4.2, we go beyond the $L^{q}$-norm in the space variable. We see in Theorem 4.6 how the assumptions on the initial conditions and on $\dot{F}$ imply that the fractional Sobolev norm in the space variable of the solution of equation (1.1) is finite. The analysis is carried out at the level 
of the s.p.d.e. driven by the smoothed kernel $G_{n}$, and then transferred to the solution of equation (1.1) by means of the properties on the fractional Sobolev norm of the contribution of the initial conditions (Lemma 4.4), an approximation result proved in Proposition 4.3 and Fatou's lemma. With the Sobolev embedings, we obtain the Hölder continuity property in the space variable of the sample paths.

In Section 4.3, we prove regularity in time using the classical approach based on Kolmogorov's continuity criterion. We fix a bounded domain $D \subset$ $\mathbb{R}^{3}$ and we first study the Hölder continuity in time, uniformly in $x \in D$, of the contribution of the initial conditions (Lemma 4.9). Secondly, we find upper bounds for the $L^{q}(\Omega)$ norm of increments in time of the solution of (1.1), uniformly in $x \in D$ (Theorem 4.10). We end up with the joint Hölder continuity in space and in time stated in Theorem 4.11. In particular, these results are non-trivial even for the deterministic inhomogeneous three-dimensional wave equation (see Remark 4.12).

In Section 5, we check the sharpness of the results proved in Section 4 by considering the most simple example consisting of an equation with vanishing initial conditions and coefficient $b$, and constant coefficient $\sigma$. In this case, the solution is a stationary Gaussian process and all the information about the sample paths is contained in the covariance function. From the results of Section 4, we already have upper bounds on $L^{q}$-norms of increments of the solution. We complete the analysis by obtaining sharp lower bounds for these increments; this requires precise estimates of integrals related to (1.4).

The last section of the article, Section 6 , gathers the somewhat technical but crucial sharp estimates on integrals of increments of a class of generalized Riesz potentials that also involve the smoothed version of the fundamental solution of the wave equation.

\section{The fundamental solution of the wave equa- tion and the covariance function}

The first part of this section is devoted to introducing the smoothing of the fundamental solution of the wave equation used throughout the paper. We prove some of its properties as well as some of the properties of the fundamental solution itself. In the second part, we obtain expressions for first and second order increments of the covariance function. Informally, these express the covariance function as a fractional integral of its fractional derivative; they are proved by applying the semigroup property of the Riesz kernels. 


\subsection{Some properties of the fundamental solution and its regularisations}

Let $d \geq 1$ and $\psi: \mathbb{R}^{d} \rightarrow \mathbb{R}_{+}$be a function in $C^{\infty}\left(\mathbb{R}^{d}\right)$ with support included in $B_{1}(0)$ and such that $\int_{\mathbb{R}^{d}} \psi(x) d x=1\left(B_{r}(x)\right.$ denotes the open ball centered at $x \in \mathbb{R}^{d}$ with radius $r \geq 0$ ). For any $\left.\left.t \in\right] 0,1\right]$ and $n \geq 1$, we define

$$
\psi_{n}(t, x)=\left(\frac{n}{t}\right)^{d} \psi\left(\frac{n}{t} x\right)
$$

and

$$
G_{n}(t, x)=\left(\psi_{n}(t, \cdot) * G(t)\right)(x),
$$

where " $*$ " denotes the convolution operation in the spatial variable. Observe that $\int_{\mathbb{R}^{d}} \psi_{n}(t, x) d x=1$ and

$$
\operatorname{supp} G_{n}(t, \cdot) \subset B_{t\left(1+\frac{1}{n}\right)}(0) \text {. }
$$

The following elementary scaling property plays an important role in the study of regularity properties in time of the stochastic integral. Its proof is included for convenience of the reader.

Lemma 2.1 Let $d=3$. For any $s, t \in[0, T]$ and $v_{0} \in C\left(\mathbb{R}^{3}\right)$,

$$
\int_{\mathbb{R}^{3}} G(s, d u) v_{0}(u)=\frac{s}{t} \int_{\mathbb{R}^{3}} G(t, d u) v_{0}\left(\frac{s}{t} u\right),
$$

and for any $x \in \mathbb{R}^{3}$,

$$
G_{n}\left(t, \frac{t}{s} x\right)=\left(\frac{s}{t}\right)^{2} G_{n}(s, x)
$$

Proof. The first equality follows from the fact that the transformation $u \mapsto \frac{s}{t} u$ maps $G(t, \cdot)$ onto $\frac{t}{s} G(s, \cdot)$.

The change of variables $y \mapsto \frac{t}{s} y$ yields

$$
\begin{aligned}
G_{n}\left(t, \frac{t}{s} x\right) & =\int_{\mathbb{R}^{3}} G(t, d y) \psi_{n}\left(t, \frac{t}{s} x-y\right) \\
& =\int_{\mathbb{R}^{3}} G(s, d y) \psi_{n}\left(t, \frac{t}{s}(x-y)\right) \frac{t}{s} .
\end{aligned}
$$

Since

$$
\left.\psi_{n}\left(t, \frac{t}{s}(x-y)\right)=\left(\frac{n}{t}\right)^{3} \psi\left(\frac{n}{s}(x-y)\right)=\psi_{n}(s, x-y)\right)\left(\frac{s}{t}\right)^{3},
$$


it follows that

$$
\begin{aligned}
G_{n}\left(t, \frac{t}{s} x\right) & \left.=\int_{\mathbb{R}^{3}} G(s, d y) \psi_{n}(s, x-y)\right)\left(\frac{s}{t}\right)^{2} \\
& =\left(\frac{s}{t}\right)^{2} G_{n}(s, x) .
\end{aligned}
$$

This proves the lemma.

We recall the following integrability condition of the fundamental solution of the wave equation, valid for any $\beta \in] 0,2[$ :

$$
\sup _{t \in[0, T]} \int_{\mathbb{R}^{d}} \frac{|\mathcal{F} G(t)(\xi)|^{2}}{|\xi|^{d-\beta}} d \xi \leq C\left(1+T^{2}\right) .
$$

Indeed,

$$
\mathcal{F} G(t)(\xi)=|\xi|^{-1} \sin (t|\xi|)
$$

(see [31]), and therefore

$$
\int_{\mathbb{R}^{d}} \frac{|\mathcal{F} G(t)(\xi)|^{2}}{|\xi|^{d-\beta}} d \xi \leq I_{1}(t)+I_{2}(t)
$$

where

$$
I_{1}(t)=\int_{|\xi| \leq 1} \frac{t^{2}}{|\xi|^{d-\beta}} d \xi \leq C_{1} T^{2}, \quad I_{2}(t)=\int_{|\xi|>1} \frac{d \xi}{|\xi|^{d+2-\beta}} d \xi \leq C_{2} .
$$

A similar property holds for $G_{n}$. In fact, since $\left|\mathcal{F} \psi_{n}(t)(\xi)\right| \leq 1$,

$$
\left|\mathcal{F} G_{n}(t)(\xi)\right|=\left|\mathcal{F} \psi_{n}(t)(\xi)\right||\mathcal{F} G(t)(\xi)| \leq|\mathcal{F} G(t)(\xi)|
$$

and therefore

$$
\sup _{n \geq 1} \sup _{t \in[0, T]} \int_{\mathbb{R}^{d}} \frac{\left|\mathcal{F} G_{n}(t)(\xi)\right|^{2}}{|\xi|^{d-\beta}} d \xi \leq C\left(1+T^{2}\right)
$$

The next statement gives a more precise result than (2.4).

Lemma 2.2 For any $t \in[0, T]$ and $\beta \in] 0,2[$,

$$
\int_{0}^{t} d s \int_{\mathbb{R}^{d}} \frac{|\mathcal{F} G(s)(\xi)|^{2}}{|\xi|^{d-\beta}} d \xi \leq C t^{3-\beta}
$$


Proof. By Fubini's theorem, and using the change of variables $w=t \xi$, we see that

$$
\begin{aligned}
\int_{0}^{t} d s \int_{\mathbb{R}^{d}} \frac{|\mathcal{F} G(s)(\xi)|^{2}}{|\xi|^{d-\beta}} d \xi & =\int_{\mathbb{R}^{d}} \frac{d \xi}{|\xi|^{d+2-\beta}} \int_{0}^{t} \frac{1-\cos (2 s|\xi|)}{2} d s \\
& =\int_{\mathbb{R}^{d}} \frac{d \xi}{|\xi|^{d+2-\beta}}\left(\frac{t}{2}-\frac{\sin (2 t|\xi|}{4|\xi|}\right) \\
& =t^{3-\beta} J,
\end{aligned}
$$

where

$$
J=\int_{\mathbb{R}^{d}} \frac{d w}{|w|^{d+2-\beta}}\left(\frac{1}{2}-\frac{\sin (2|w|)}{4|w|}\right) .
$$

Note that $J<\infty$. Indeed, $J \leq J_{1}+J_{2}$, where

$$
J_{1}=\int_{|w|>1} \frac{d w}{|w|^{d+2-\beta}}, \quad J_{2}=\int_{|w| \leq 1} \frac{d w}{|w|^{d+2-\beta}}\left(\frac{1}{2}-\frac{\sin (2|w|)}{4|w|}\right) .
$$

Clearly, $J_{1}<\infty$. For $J_{2}$, since $\sin (2|w|)=2|w|-\frac{2^{3}}{3 !} \cos (\zeta)|w|^{3}$, with $\zeta \in$ ]0, $|w|[$,

$$
J_{2} \leq C \int_{|w| \leq 1} \frac{d w}{|w|^{d-\beta}}<\infty
$$

This establishes (2.8).

Lemma 2.3 For any $b>0$ and $\beta \in] 0,2[$ such that $\beta+b \in] 0,3[$,

$$
\sup _{t \in[0, T]} \int_{0}^{t} \frac{d s}{s^{b}} \int_{\mathbb{R}^{d}} \frac{|\mathcal{F} G(s)(\xi)|^{2}}{|\xi|^{d-\beta}} d \xi<\infty .
$$

Proof. The change of variables $\xi \mapsto s \xi$ shows that the integral in (2.9) is equal to

$$
\int_{0}^{t} d s s^{2-(\beta+b)} \int_{\mathbb{R}^{d}} \frac{\sin ^{2}|\xi|}{|\xi|^{d+2-\beta}} d \xi .
$$

The inner integral is finite for $\beta \in] 0,2[$. Therefore (2.9) holds when $\beta+b<3$. 


\subsection{The covariance function and Riesz kernels}

We assume that the covariance measure of the noise is absolutely continuous with respect to Lebesgue measure, that is, $\Gamma(d x)=f(x) d x$. In addition, we suppose that $f$ satisfies the following assumption.

Assumption 2.4 There is $\beta \in] 0,2[$ and $\delta \in] 0,1]$ such that

$$
f(x)=\varphi(x) k_{\beta}(x),
$$

where $k_{\beta}(x)=|x|^{-\beta}, x \in \mathbb{R}^{d} \backslash\{0\}$, and $\varphi$ is bounded and positive, $\varphi \in C^{1}\left(\mathbb{R}^{d}\right)$ and $\nabla \varphi \in C_{b}^{\delta}\left(\mathbb{R}^{d}\right)$ (the space of bounded and Hölder continuous functions with exponent $\delta)$.

Example 2.5 (a) The basic case is when $\varphi \equiv 1$. In this case, $f \equiv k_{\beta}$ is termed a Riesz kernel. We recall that $k_{\beta}=c_{d, \beta} \mathcal{F} k_{d-\beta}$ [30, Chapter V].

(b) Another possibility is $\varphi(x)=\exp \left(-\sigma^{2}|x|^{2} / 2\right)$. In this case, $f(x)$ is indeed a covariance function, since $f=\mathcal{F}\left(k_{d-\beta} * \psi\right)$, where $\psi(\xi)=$ $\left(2 \pi \sigma^{2}\right)^{-3 / 2} \exp \left(-|\xi|^{2} /\left(2 \sigma^{2}\right)\right)$. The parameter $\delta$ in Assumption 2.4 can be set to 1. Condition (1.4) is satisfied since $\beta \in] 0,2]$. Indeed,

$$
\begin{aligned}
\int_{\mathbb{R}^{d}} d \xi\left(k_{d-\beta} * \psi\right)(\xi)|\mathcal{F} G(t)(\xi)|^{2} & =\int_{\mathbb{R}^{d}} d z \psi(z) \int_{\mathbb{R}^{d}} d \xi k_{d-\beta}(\xi)|\mathcal{F} G(t)(\xi+\eta)|^{2} \\
& \leq \sup _{\eta \in \mathbb{R}^{d}} \int_{\mathbb{R}^{d}} d \xi k_{d-\beta}(\xi)|\mathcal{F} G(t)(\xi+\eta)|^{2},
\end{aligned}
$$

and the right-hand side is finite when $\beta \in] 0,2]$ : see [7, Lemma 8].

The Riesz potentials $I_{a}$ associated with the function $k_{\beta}(x)$ are defined by

$$
\left(I_{a} \varphi\right)(x)=\frac{1}{\gamma(a)} \int_{\mathbb{R}^{d}}|x-y|^{-d+a} \varphi(y) d y,
$$

for $\left.\varphi \in \mathcal{S}\left(\mathbb{R}^{d}\right), a \in\right] 0, d\left[\right.$ and $\gamma(a)=\pi^{d / 2} 2^{a} \Gamma\left(\frac{a}{2}\right) / \Gamma\left(\frac{d-a}{2}\right)$. Riesz potentials can be interpreted as fractional integrals and have the semigroup property

$$
\left.I_{a+b} \varphi=I_{a}\left(I_{b} \varphi\right), \varphi \in \mathcal{S}\left(\mathbb{R}^{d}\right), a+b \in\right] 0, d[
$$

(see [30, p.118]). This property implies in particular that

$$
|x-y|^{-d+(a+b)}=\int_{\mathbb{R}^{d}} d z|x-z|^{-d+a}|z-y|^{-d+b},
$$

provided $a+b \in] 0, d[$. This equality can be informally interpreted by saying that $|\cdot|^{-d+(a+b)}$ is the fractional integral of order $a$ of $|\cdot|^{-d+b}$, which is 
natural since $(-\Delta)^{a / 2}\left(|\cdot|^{-d+(a+b)}\right)=|\cdot|^{-d+b}$ as can be checked by taking Fourier transforms.

We will make heavy use of properties of first and second order increments of Riesz kernels. For a function $f: \mathbb{R}^{d} \rightarrow \mathbb{R}$, we set

$$
\begin{aligned}
D f(u, x) & =f(u+x)-f(u), \\
D^{2} f(u, x) & =f(u-x)-2 f(u)+f(u+x), \\
\bar{D}^{2} f(u, x, y) & =f(u+x+y)-f(u+x)-f(u+y)+f(u) .
\end{aligned}
$$

Notice that $D^{2} f(u, x)=\bar{D}^{2} f(u-x, x, x)$.

Lemma 2.6 Fix $\left.u, x, y \in \mathbb{R}^{d}, a+b \in\right] 0, d[$. The following properties hold:

(a) For any $c \in \mathbb{R}$,

$$
D k_{d-a-b}(u, c x)=|c|^{b} \int_{\mathbb{R}^{d}} d w k_{d-a}(u-c w) D k_{d-b}(w, x) .
$$

(b) For any $b \in] 0,1\left[\right.$ and any vector $e \in \mathbb{R}^{d}$ with $|e|=1$,

$$
\int_{\mathbb{R}^{d}} d w\left|D k_{d-b}(w, e)\right|<\infty
$$

(c) Set $e=\frac{x}{|x|}, x \in \mathbb{R}^{d}$. Then

$$
\left|D^{2} k_{d-a-b}(u, x)\right| \leq|x|^{b} \int_{\mathbb{R}^{d}} d w k_{d-a}(u-|x| w)\left|D^{2} k_{d-b}(w, e)\right| .
$$

(d) For any $b \in] 0,2\left[\right.$ and each $e \in \mathbb{R}^{d}$ with $|e|=1$,

$$
\int_{\mathbb{R}^{d}} d w\left|D^{2} k_{d-b}(w, e)\right|<\infty .
$$

(e)

$$
\bar{D}^{2} k_{d-a-b}(u, c x, c y) \leq|c|^{b} \int_{\mathbb{R}^{d}} d w k_{d-a}(u-c w)\left|\bar{D}^{2} k_{d-b}(w, x, y)\right|
$$


Proof. (a) From (2.10), we obtain

$$
D k_{d-a-b}(u, c x)=\int_{\mathbb{R}^{d}} d z k_{d-a}(u-z) D k_{d-b}(z, c x) .
$$

Set $z=c w$ to see that this is equal to

$$
\begin{aligned}
& |c|^{d} \int_{\mathbb{R}^{d}} d w k_{d-a}(u-c w) D k_{d-b}(c w, c x) \\
& \quad=|c|^{b} \int_{\mathbb{R}^{d}} d w k_{d-a}(u-c w) D k_{d-b}(w, x),
\end{aligned}
$$

which proves (a).

We now check (b). Set

$$
I=\int_{\mathbb{R}^{d}} d w\left|D k_{d-b}(w, e)\right|
$$

and consider the decomposition $I=I_{1}+I_{2}$, where $I_{1}$ (resp. $I_{2}$ ) is the integral of the same expression but over $B_{2}(0)$ (resp. $\left.B_{2}(0)^{c}\right)$ instead of $\mathbb{R}^{d}$. Then

$$
I_{1} \leq 2 \int_{B_{3}(0)} d w|w|^{-d+b} \leq C \int_{0}^{3} \rho^{b-1} d \rho<\infty,
$$

if $b>0$. As for $I_{2}$, we write

$$
\begin{aligned}
I_{2} & =\int_{|w|>2} d w \mid \int_{0}^{1} d \lambda \frac{d}{d \lambda}\left(|w-\lambda e|^{-d+b}\right) \\
& \leq \int_{0}^{1} d \lambda \int_{|w|>2} d w|w-\lambda e|^{-d+b-1} \\
& \leq C \int_{0}^{1} d \lambda \int_{1}^{\infty} \rho^{b-2} d \rho,
\end{aligned}
$$

which is finite if $b<1$.

The proof of (c) is analogous to that of (a). It suffices to apply the identity (2.10) twice, since $D^{2} k_{d-a-b}(u, x)=D k_{d-a-b}(u, x)+D k_{d-a-b}(u,-x)$, and to use the change of variables $z=|x| w$.

Let us now prove (d). Observe that $D^{2} k_{d-b}(\omega, e)=k_{d-b}(w \pm e)-2 k_{d-b}(w)$ and that the integrals

$$
\int_{|w| \leq 2} d w|w|^{-d+b}, \quad \int_{|w| \leq 2} d w|e \pm w|^{-d+b},
$$

converge for each $b>0$.

We next study $\int_{|w|>2} d w\left|D^{2} k_{d-b}(w, e)\right|$. Set

$$
\Phi(\lambda, \mu)=k_{d-b}(w-(\lambda-\mu) e), \lambda, \mu \in[0,1] .
$$


Then

$$
D^{2} k_{d-b}(w, e)=\int_{0}^{1} d \lambda \int_{0}^{1} d \mu \frac{\partial^{2} \Phi}{\partial \lambda \partial \mu}(\lambda, \mu) .
$$

Elementary computations lead to

$$
\left|\frac{\partial^{2} \Phi}{\partial \lambda \partial \mu}(\lambda, \mu)\right| \leq C k_{d-b+2}(w-(\lambda-\mu) e) .
$$

Therefore, by Fubini's theorem,

$$
\int_{|w|>2} d w\left|D^{2} k_{d-b}(w, e)\right| \leq C \int_{0}^{1} d \lambda \int_{0}^{1} d \mu \int_{|w|>2} d w|w-(\lambda-\mu) e|^{-d+b-2} .
$$

The integral $\int_{|w|>2} d w|w-(\lambda-\mu) e|^{-d+b-2}$ converges for any $b<2$. Consequently, (d) is proved.

The proof of (e) is analogous to that of (a). It suffices to apply the identity (2.10) four times, and to use the change of variables $z=c w$.

Lemma 2.6 is the basis for the main technical estimates of this paper, whose statements and proofs are deferred to Section 6 .

\section{Hölder-Sobolev regularity of the stochastic integral}

In this section, we consider the extension of the stochastic integral given in Section 2 of [7] in the particular case where $G$ is the fundamental solution of the wave equation in spatial dimension $d=3$, defined in (1.3). More precisely, let $\{Z(s), s \in[0, T]\}$ be an $\mathcal{F}_{s}$-measurable, $L^{2}\left(\mathbb{R}^{3}\right)$-valued stochastic process that is mean-square continuous. Let $F$ be the Gaussian process with covariance measure $\Gamma$ and covariance functional as in (1.2). Assume that its spectral measure $\mu=\mathcal{F}^{-1} \Gamma$ satisfies condition (1.4).

As it has been proved in [5], the process $F$ can be extended to a worthy martingale measure in the sense given in [32]. We shall denote by $M=$ $\left(M_{t}(A), t \geq 0, A \in \mathcal{B}_{b}\left(\mathbb{R}^{3}\right)\right)$ this extension. The relationship between $F$ and $M$ is

$$
F(\psi)=\int_{\mathbb{R}_{+} \times \mathbb{R}^{3}} \psi(t, x) M(d t, d x),
$$

for all $\psi \in \mathcal{D}\left(\mathbb{R}^{d+1}\right)$. The covariance measure $Q$ and dominating measure $K$ of $M$ are

$$
Q(A \times B \times] s, t])=(t-s) \int_{\mathbb{R}^{3}} d x \int_{\mathbb{R}^{3}} d y 1_{A}(x) f(x-y) 1_{B}(y)
$$


and $K \equiv Q$.

Then, following Theorem 6 in [7], the stochastic integral

$$
v_{G, Z}^{t}=\int_{0}^{t} \int_{\mathbb{R}^{3}} G(t-s, \cdot-y) Z(s, y) M(d s, d y)
$$

is well-defined as a random vector in $L^{2}\left(\Omega ; L^{2}\left(\mathbb{R}^{3}\right)\right)$ and has the isometry property

$E\left(\left\|v_{G, Z}^{t}\right\|_{L^{2}\left(\mathbb{R}^{3}\right)}^{2}\right)=\int_{0}^{t} d s \int_{\mathbb{R}^{3}} d \xi E\left(|\mathcal{F} Z(s)(\xi)|^{2}\right) \int_{\mathbb{R}^{3}} \mu(d \eta)|\mathcal{F} G(t-s)(\xi-\eta)|^{2}$.

It also satisfies the bound

$E\left(\left\|v_{G, Z}^{t}\right\|_{L^{2}\left(\mathbb{R}^{3}\right)}^{2}\right) \leq \int_{0}^{t} d s E\left(\|Z(s)\|_{L^{2}\left(\mathbb{R}^{3}\right)}^{2}\right) \sup _{\xi \in \mathbb{R}^{3}} \int_{\mathbb{R}^{3}} \mu(d \eta)|\mathcal{F} G(t-s)(\xi-\eta)|^{2}$.

Let $\mathcal{O}$ be a bounded or unbounded open subset of $\mathbb{R}^{3}, p \in[1, \infty[, \gamma \in] 0,1[$. Define

$$
\|g\|_{\gamma, p, \mathcal{O}}=\left(\int_{\mathcal{O}} d x \int_{\mathcal{O}} d y \frac{|g(x)-g(y)|^{p}}{|x-y|^{3+\gamma p}}\right)^{1 / p} .
$$

When $\mathcal{O}=\mathbb{R}^{3}$, we write $\|g\|_{\gamma, p}$ instead of $\|g\|_{\gamma, p, \mathbb{R}^{3}}$.

We denote by $W^{\gamma, p}(\mathcal{O})$ the Banach space consisting of functions $g: \mathbb{R}^{3} \rightarrow$ $\mathbb{R}$ such that

$$
\|g\|_{W^{\gamma, p}(\mathcal{O})}:=\|g\|_{L^{p}(\mathcal{O})}+\|g\|_{\gamma, p, \mathcal{O}}
$$

is finite. The spaces $W^{\gamma, p}(\mathcal{O})$ are the fractional Sobolev spaces (see for instance [1], [28]).

Given a bounded set $K \subset \mathbb{R}^{3}$ and $\varepsilon>0$, we let $K^{\varepsilon}$ be the open set

$$
K^{\varepsilon}=\left\{x \in \mathbb{R}^{3}: \exists z \in K \text { such that }|x-z|<\varepsilon\right\} .
$$

\subsection{Regularity in space}

The following result concerns the Sobolev regularity of the stochastic integral in the space variable. We assume that $\Gamma(d x)$ is of the form $\Gamma(d x)=f(x) d x$, where $f(x)$ is as in (1.5), and there is $\beta \in] 0,2[$ and $\delta \in] 0,1[$ such that Assumption 2.4 is satisfied.

Theorem 3.1 Fix $T>0, q \in] 3, \infty\left[\right.$ and a bounded domain $\mathcal{O} \subset \mathbb{R}^{3}$. Suppose that $\tau_{q}(\beta, \delta):=\left(\frac{2-\beta}{2} \wedge \frac{1+\delta}{2}\right)-\frac{3}{q}>0$ and fix $\left.\gamma \in\right] 0,1[, \rho \in] 0, \tau_{q}(\beta, \delta) \wedge \gamma[$. Consider a stochastic process $Z$ such that for some fixed $t \in[0, T]$,

$$
\int_{0}^{t} d s E\left(\|Z(s)\|_{W^{\gamma, q}\left(\mathcal{O}^{t-s}\right)}^{q}\right)<\infty .
$$


There is $C<\infty$ (depending on $\rho$ but not on $Z$ ) such that

$$
E\left(\left\|v_{G, Z}^{t}\right\|_{W^{\rho, q}(\mathcal{O})}^{q}\right) \leq C \int_{0}^{t} d s E\left(\|Z(s)\|_{W^{\rho, q}\left(\mathcal{O}^{t-s}\right)}^{q}\right) .
$$

The main ingredient in the proof of this theorem is the next lemma, which will also be used in the proof of Theorem 4.6 in the next section.

Lemma 3.2 Fix $q \in] 3, \infty\left[\right.$ such that $\tau_{q}(\beta, \delta)>0$. Fix $\left.\rho \in\right] 0, \tau_{q}(\beta, \delta)[$, a compact set $K \subset \mathbb{R}^{3}$ and a bounded domain $\mathcal{O} \subset K$. Let $G_{n}$ be given in (2.1). For any $\alpha \in] 0,(2-\beta) \wedge 1\left[\right.$ satisfying $\frac{\alpha}{2}-\frac{3}{q}<\rho$, there is a finite constant $C=C(T, \rho, \alpha, K)$ such that for every $t \in[0, T]$ and $n \geq 1$,

$$
\begin{aligned}
E & \left(\left\|v_{G_{n}, Z}^{t}\right\|_{\rho, q, \mathcal{O}}^{q}\right) \leq C \int_{0}^{t} d s\left(E\left(\|Z(s)\|_{W^{\rho, q}\left(\mathcal{O}^{(t-s)\left(1+\frac{1}{n}\right)}\right)}^{q}\right)\right. \\
& \left.+\left(E\left(\|Z(s)\|_{L^{q}\left(\mathcal{O}^{(t-s)\left(1+\frac{1}{n}\right)}\right)}^{q}\right) E\left(\|Z(s)\|_{2 \rho-\alpha+\frac{3}{q}, q, \mathcal{O}^{(t-s)\left(1+\frac{1}{n}\right)}}^{q}\right)\right)^{\frac{1}{2}}\right) .
\end{aligned}
$$

In addition,

$$
E\left(\left\|v_{G_{n}, Z}^{t}\right\|_{\rho, q, \mathcal{O}}^{q}\right) \leq C \int_{0}^{t} d s E\left(\|Z(s)\|_{W^{\rho, q}\left(\mathcal{O}^{(t-s)\left(1+\frac{1}{n}\right)}\right)}^{q}\right) .
$$

Proof: Set $p=q / 2$ and fix $t \in] 0, T]$. Note that $v_{G_{n}, Z}^{t}(x)$ is a Walsh stochastic integral and

$$
\begin{aligned}
v_{G_{n}, Z}^{t}(x) & -v_{G_{n}, Z}^{t}(y) \\
& =\int_{0}^{t} d s \int_{\mathbb{R}^{3}}\left(G_{n}(t-s, x-u)-G_{n}(t-s, y-u)\right) Z(s, u) M(d s, d u) .
\end{aligned}
$$

Burkholder's inequality yields

$$
\begin{aligned}
& E\left(\left|v_{G_{n}, Z}^{t}(x)-v_{G_{n}, Z}^{t}(y)\right|^{q}\right) \\
& \leq C E\left(\mid \int_{0}^{t} d s \int_{\mathbb{R}^{3}} d u \int_{\mathbb{R}^{3}} d v Z(s, u) f(u-v) Z(s, v)\right. \\
& \times\left(G_{n}(t-s, x-u)-G_{n}(t-s, y-u)\right) \\
&\left.\times\left.\left(G_{n}(t-s, x-v)-G_{n}(t-s, y-v)\right)\right|^{p}\right) \\
&= C E\left(\left|I_{n}^{t}(x, x)-I_{n}^{t}(x, y)-I_{n}^{t}(y, x)+I_{n}^{t}(y, y)\right|\right)^{p},
\end{aligned}
$$


with

$$
I_{n}^{t}(x, y)=\int_{0}^{t} d s \int_{\mathbb{R}^{3}} d u \int_{\mathbb{R}^{3}} d v g_{n}(t, s, x, y, u, v) Z(s, u) f(u-v) Z(s, v)
$$

and

$$
g_{n}(t, s, x, y, u, v)=G_{n}(t-s, x-u) G_{n}(t-s, y-v) .
$$

After the change of variables $s \mapsto t-s$ and the spatial transformation $(u, v) \mapsto$ $(x-u, y-v)$, we obtain

$$
I_{n}^{t}(x, y)=\int_{0}^{t} d s \int_{\mathbb{R}^{3}} d u \int_{\mathbb{R}^{3}} d v G_{n}(s, u) G_{n}(s, v) h(t, s, x, y, u, v),
$$

where

$$
h(t, s, x, y, u, v)=Z(t-s, x-u) f(x-y-u+v) Z(t-s, y-v) .
$$

Using these expressions and rearranging terms, it is straightforward to check that

$$
I_{n}^{t}(x, x)-I_{n}^{t}(x, y)-I_{n}^{t}(y, x)+I_{n}^{t}(y, y)=\sum_{i=1}^{4} J_{i, n}^{t}(x, y),
$$

where, for $i=1, \ldots, 4$,

$$
J_{i, n}^{t}(x, y)=\int_{0}^{t} d s \int_{\mathbb{R}^{3}} d u \int_{\mathbb{R}^{3}} d v G_{n}(s, u) G_{n}(s, v) h_{i}(t, s, x, y, u, v),
$$

and, using the notation in (2.11),

$$
\begin{aligned}
h_{1}(t, s, x, y, u, v)=f(y-x+v-u)(Z(t-s, x-u)-Z(t-s, y-u)) \\
\times(Z(t-s, x-v)-Z(t-s, y-v)), \\
h_{2}(t, s, x, y, u, v)=D f(v-u, x-y) Z(t-s, x-u) \\
\times(Z(t-s, x-v)-Z(t-s, y-v)), \\
h_{3}(t, s, x, y, u, v)=D f(v-u, y-x) Z(t-s, y-v) \\
\times(Z(t-s, x-u)-Z(t-s, y-u)), \\
h_{4}(t, s, x, y, u, v)=-D^{2} f(v-u, x-y) Z(t-s, x-u) Z(t-s, x-v) .
\end{aligned}
$$

Consequently, setting $\bar{\rho}=\rho+\frac{3}{q}$ and

$$
T_{n}(t, \mathcal{O})=E\left(\left\|v_{G_{n}, Z}^{t}\right\|_{\rho, q, \mathcal{O}}^{q}\right),
$$


we have

$$
T_{n}(t, \mathcal{O}) \leq C \sum_{i=1}^{4} T_{n}^{i}(t, \mathcal{O})
$$

where

$$
T_{n}^{i}(t, \mathcal{O})=\int_{\mathcal{O}} d x \int_{\mathcal{O}} d y \frac{E\left(\left|J_{i, n}^{t}(x, y)\right|^{p}\right)}{|x-y|^{q \bar{\rho}}}
$$

Set

$$
\mu_{n}^{1}(x, y)=\sup _{s \in[0, T]} \int_{\mathbb{R}^{3}} d u \int_{\mathbb{R}^{3}} d v G_{n}(s, u) G_{n}(s, v) f(y-x+v-u) .
$$

Notice that, since $f(x)=\varphi(x) k_{\beta}(x)$ with $\varphi$ bounded (see Assumption 2.4) and $G_{n} \geq 0$,

$$
\sup _{n, x, y} \mu_{n}^{1}(x, y) \leq \int_{\mathbb{R}^{3}} \frac{|\mathcal{F} G(s)(\xi)|^{2}}{|\xi|^{3-\beta}} d \xi<\infty,
$$

for any $\beta \in] 0,2[$ (see (2.4)).

Therefore, Hölder's inequality implies that

$$
\begin{aligned}
E\left(\left|J_{1, n}^{t}(x, y)\right|^{p}\right) \leq & \left(T \mu_{n}^{1}(x, y)\right)^{p-1} \\
& \times E\left(\int_{0}^{t} d s \int_{\mathbb{R}^{3}} d u \int_{\mathbb{R}^{3}} d v G_{n}(s, u) G_{n}(s, v) f(y-x+v-u)\right. \\
& \times|Z(t-s, x-u)-Z(t-s, y-u)|^{p} \\
& \left.\times|Z(t-s, x-v)-Z(t-s, y-v)|^{p}\right) .
\end{aligned}
$$

The function $(s, x, y, u, v) \mapsto G_{n}(s, u) G_{n}(s, v) f(y-x+v-u)$ is the density of a finite measure on $[0, T] \times \mathcal{O} \times \mathcal{O} \times \mathbb{R}^{3} \times \mathbb{R}^{3}$. Indeed,

$$
\begin{aligned}
& \sup _{s \in[0, T]} \int_{\mathcal{O}} d x \int_{\mathcal{O}} d y \int_{\mathbb{R}^{3}} d u \int_{\mathbb{R}^{3}} d v G_{n}(s, u) G_{n}(s, v) f(y-x+v-u) \\
& \quad \leq|\mathcal{O}|^{2} \sup _{n, x, y} \mu_{n}^{1}(x, y) \leq C
\end{aligned}
$$

where $|\mathcal{O}|$ denotes the volume of $\mathcal{O}$. 
Applying the Cauchy-Schwarz inequality with respect to this measure, we see that $T_{n}^{1}(t, \mathcal{O}) \leq C\left(T_{n}^{1,1}(t, \mathcal{O}) T_{n}^{1,2}(t, \mathcal{O})\right)^{\frac{1}{2}}$, with

$$
\begin{aligned}
T_{n}^{1,1}(t, \mathcal{O})= & \int_{0}^{t} d s \int_{\mathcal{O}} d x \int_{\mathcal{O}} d y \int_{\mathbb{R}^{3}} d u \int_{\mathbb{R}^{3}} d v G_{n}(s, u) G_{n}(s, v) f(y-x+v-u) \\
& \times \frac{E\left(|Z(t-s, x-u)-Z(t-s, y-u)|^{2 p}\right)}{|x-y|^{q \bar{\rho}}}, \\
T_{n}^{1,2}(t, \mathcal{O})= & \int_{0}^{t} d s \int_{\mathcal{O}} d x \int_{\mathcal{O}} d y \int_{\mathbb{R}^{3}} d u \int_{\mathbb{R}^{3}} d v G_{n}(s, u) G_{n}(s, v) f(y-x+v-u) \\
& \times \frac{E\left(|Z(t-s, x-v)-Z(t-s, y-v)|^{2 p}\right)}{|x-y|^{q \bar{\rho}}} .
\end{aligned}
$$

Consider the change of variables defined by $\bar{x}=x-u, \bar{y}=y-u$. We notice that for any $s \in[0, T]$ and $n \geq 1$, the support of the function $G_{n}(s)$ is included in the ball centered at zero with radius $s k_{n}$, where $k_{n}=1+\frac{1}{n}$. Therefore, the domain of the new variables $\bar{x}, \bar{y}$ is included in $\mathcal{O}^{s k_{n}}$. Hence,

$$
\begin{aligned}
& T_{n}^{1,1}(t, \mathcal{O}) \leq \int_{0}^{t} d s \int_{\mathcal{O}^{s k_{n}}} d \bar{x} \int_{\mathcal{O}^{s k_{n}}} d \bar{y} E\left(\left|\frac{Z(t-s, \bar{x})-Z(t-s, \bar{y})}{|\bar{x}-\bar{y}|^{\bar{\rho}}}\right|^{q}\right) \\
& \times \int_{\mathbb{R}^{3}} d u \int_{\mathbb{R}^{3}} d v G_{n}(s, u) G_{n}(s, v) f(\bar{y}-\bar{x}+v-u) \\
& \leq \sup _{n, \bar{x}, \bar{y}} \mu_{n}^{1}(\bar{x}, \bar{y}) \\
& \times \int_{0}^{t} d s \int_{\mathcal{O}^{s k_{n}}} d \bar{x} \int_{\mathcal{O}^{s k_{n}}} d \bar{y} E\left(\left|\frac{Z(t-s, \bar{x})-Z(t-s, \bar{y})}{|\bar{x}-\bar{y}|^{\bar{\rho}}}\right|^{q}\right) \\
& \leq C \int_{0}^{t} d s E\left(\|Z(s)\|_{\rho, q, \mathcal{O}^{(t-s) k_{n}}}^{q}\right) .
\end{aligned}
$$

We deal with the term $T_{n}^{1,2}(t, \mathcal{O})$ similarly, using the change of variables $\bar{x}=x-v, \bar{y}=y-v$. We obtain the same upper bound as for $T_{n}^{1,1}(t, \mathcal{O})$. Summarising,

$$
T_{n}^{1}(t, \mathcal{O}) \leq C \int_{0}^{t} d s E\left(\|Z(s)\|_{\rho, q, \mathcal{O}^{(t-s)\left(1+\frac{1}{n}\right)}}^{q}\right) .
$$


Set

$$
\mu_{n}^{2}(x, y)=\sup _{s \in[0, T]} \int_{\mathbb{R}^{3}} d u \int_{\mathbb{R}^{3}} d v G_{n}(s, u) G_{n}(s, v)|D f(v-u, x-y)| .
$$

By Lemma 6.1,

$$
\sup _{n \geq 1} \mu_{n}^{2}(x, y) \leq C|x-y|^{\alpha},
$$

for any $\alpha \in] 0,(2-\beta) \wedge 1[$.

By Hölder's inequality, for any $\alpha \in] 0,(2-\beta) \wedge 1[$,

$$
\begin{aligned}
& \frac{E\left(\left|J_{2, n}^{t}(x, y)\right|^{p}\right)}{|x-y|^{q \bar{\rho}}} \leq\left(\sup _{n, x, y} \frac{T \mu_{n}^{2}(x, y)}{|x-y|^{\alpha}}\right)^{p-1} \\
& \quad \times \int_{0}^{t} d s \int_{\mathbb{R}^{3}} d u \int_{\mathbb{R}^{3}} d v G_{n}(s, u) G_{n}(s, v) \frac{|D f(v-u, x-y)|}{|x-y|^{\alpha}} \\
& \quad \times E\left(|Z(t-s, x-u)|^{p} \frac{|Z(t-s, x-v)-Z(t-s, y-v)|^{p}}{|x-y|^{p(2 \bar{\rho}-\alpha)}}\right) .
\end{aligned}
$$

For each $s \in[0, T]$, the function $(x, y, u, v) \mapsto G_{n}(s, u) G_{n}(s, v) \frac{|D f(v-u, x-y)|}{|x-y|^{\alpha}}$ is the density of a finite measure on the set $\mathcal{O} \times \mathcal{O} \times \mathbb{R}^{3} \times \mathbb{R}^{3}$. In fact,

$$
\begin{aligned}
& \int_{\mathcal{O}} d x \int_{\mathcal{O}} d y \int_{\mathbb{R}^{3}} d u \int_{\mathbb{R}^{3}} d v G_{n}(s, u) G_{n}(s, v) \frac{|D f(v-u, x-y)|}{|x-y|^{\alpha}} \\
& \leq|\mathcal{O}|^{2} \sup _{n, x, y} \frac{\mu_{n}^{2}(x, y)}{|x-y|^{\alpha}} \leq C .
\end{aligned}
$$

By the Cauchy-Schwartz inequality,

$$
T_{n}^{2}(t, \mathcal{O}) \leq C \int_{0}^{t} d s\left(T_{n}^{2,1}(s, \mathcal{O}) T_{n}^{2,2}(s, \mathcal{O})\right)^{\frac{1}{2}},
$$


with the terms $T_{n}^{2,1}(s, \mathcal{O})$ and $T_{n}^{2,2}(s, \mathcal{O})$ defined as follows:

$$
\begin{aligned}
T_{n}^{2,1}(s, \mathcal{O})= & \int_{\mathcal{O}} d x \int_{\mathcal{O}} d y \int_{\mathbb{R}^{3}} d u \int_{\mathbb{R}^{3}} d v G_{n}(s, u) G_{n}(s, v) \frac{|D f(v-u, x-y)|}{|x-y|^{\alpha}} \\
& \times E\left(|Z(t-s, x-u)|^{q}\right), \\
T_{n}^{2,2}(s, \mathcal{O})= & \int_{\mathcal{O}} d x \int_{\mathcal{O}} d y \int_{\mathbb{R}^{3}} d u \int_{\mathbb{R}^{3}} d v G_{n}(s, u) G_{n}(s, v) \frac{|D f(v-u, x-y)|}{|x-y|^{\alpha}} \\
& \times E\left(\left|\frac{Z(t-s, x-v)-Z(t-s, y-v)}{|x-y|^{2 \bar{\rho}-\alpha}}\right|^{q}\right) .
\end{aligned}
$$

Introducing the change of variables $\bar{x}=x-u, \bar{y}=y-u$ yields

$$
\begin{aligned}
T_{n}^{2,1}(s, \mathcal{O}) \leq & \int_{\mathcal{O}^{s k_{n}}} d \bar{x} \int_{\mathcal{O}^{s k_{n}}} d \bar{y} \int_{\mathbb{R}^{3}} d u \int_{\mathbb{R}^{3}} d v G_{n}(s, u) G_{n}(s, v) \\
& \times \frac{|D f(v-u, \bar{x}-\bar{y})|}{|\bar{x}-\bar{y}|^{\alpha}} E\left(|Z(t-s, \bar{x})|^{q}\right) \\
\leq & C \sup _{n, \bar{x}, \bar{y}}\left(\frac{\mu_{n}^{2}(x, y)}{|\bar{x}-\bar{y}|^{\alpha}}\right) E\left(\|Z(t-s)\|_{L^{q}\left(\mathcal{O}^{\left.s k_{n}\right)}\right)}^{q}\right) .
\end{aligned}
$$

For the term $T_{n}^{2,2}(s, \mathcal{O})$, we consider the new variables $\bar{x}=x-v, \bar{y}=y-v$. We obtain

$$
\begin{aligned}
& T_{n}^{2,2}(s, \mathcal{O}) \leq \int_{\mathcal{O}^{s k_{n}}} d \bar{x} \int_{\mathcal{O}^{s k_{n}}} d \bar{y} \int_{\mathbb{R}^{3}} d u \int_{\mathbb{R}^{3}} d v G_{n}(s, u) G_{n}(s, v) \\
& \times \frac{|D f(v-u, \bar{x}-\bar{y})|}{|\bar{x}-\bar{y}|^{\alpha}} E\left(\left|\frac{Z(t-s, \bar{x})-Z(t-s, \bar{y})}{|\bar{x}-\bar{y}|^{2 \bar{\rho}-\alpha}}\right|^{q}\right) \\
& \leq C \sup _{n, \bar{x}, \bar{y}}\left(\frac{\mu_{n}^{2}(x, y)}{|\bar{x}-\bar{y}|^{\alpha}}\right) E\left(\|Z(t-s)\|_{2 \bar{\rho}-\alpha-\frac{3}{q}, q, \mathcal{O}^{s k_{n}}}^{q}\right) .
\end{aligned}
$$

Hence, we have obtained

$$
\begin{array}{rl}
T_{n}^{2}(t, \mathcal{O}) \leq C \int_{0}^{t} & d s\left(E\left(\|Z(s)\|_{L^{q}\left(\mathcal{O}^{(t-s)\left(1+\frac{1}{n}\right)}\right)}^{q}\right)\right. \\
& \left.\times E\left(\|Z(s)\|_{2 \bar{\rho}-\alpha-\frac{3}{q}, q, \mathcal{O}^{(t-s)\left(1+\frac{1}{n}\right)}}^{q}\right)\right)^{\frac{1}{2}} .
\end{array}
$$


With the same arguments, one can find an identical upper bound for the term $T_{n}^{3}(t, \mathcal{O})$.

We continue with the analysis of $T_{n}^{4}(t, \mathcal{O})$. Set

$$
\mu_{n}^{4}(x, y)=\sup _{s \in[0, T]} \int_{\mathbb{R}^{3}} d u \int_{\mathbb{R}^{3}} d v G_{n}(s, u) G_{n}(s, v)\left|D^{2} f(v-u, x-y)\right|,
$$

where $D^{2} f$ is defined in (2.11). By Lemma 6.2, if $\left.\bar{\rho} \in\right] 0, \frac{2-\beta}{2} \wedge \frac{1+\delta}{2}[$,

$$
\sup _{n \geq 1} \mu_{n}^{4}(x, y) \leq C|x-y|^{2 \bar{\rho}}
$$

Then, Hölder's inequality yields

$$
\begin{aligned}
\frac{E\left(\left|J_{4, n}^{t}(x, y)\right|^{p}\right)}{|x-y|^{2 p \bar{\rho}}} \leq & \left(\sup _{n, x, y} \frac{T \mu_{n}^{4}(x, y)}{|x-y|^{2 \bar{\rho}}}\right)^{p-1} \\
& \times \int_{0}^{t} d s \int_{\mathbb{R}^{3}} d u \int_{\mathbb{R}^{3}} d v G_{n}(s, u) G_{n}(s, v) \\
& \times \frac{\left|D^{2} f(v-u, x-y)\right|}{|x-y|^{2 \bar{\rho}}} \\
& \times E\left(|Z(t-s, x-u)|^{p}|Z(t-s, x-v)|^{p}\right),
\end{aligned}
$$

Therefore,

$$
\begin{aligned}
T_{n}^{4}(t, \mathcal{O}) \leq & C \int_{0}^{t} d s \int_{\mathcal{O}} d x \int_{\mathcal{O}} d y \int_{\mathbb{R}^{3}} d u \int_{\mathbb{R}^{3}} d v G_{n}(s, u) G_{n}(s, v) \\
& \times \frac{\left|D^{2} f(v-u, x-y)\right|}{|x-y|^{2 \bar{\rho}}} E\left(|Z(t-s, x-u)|^{p}|Z(t-s, x-v)|^{p}\right) .
\end{aligned}
$$

Since the function $(s, x, y, u, v) \mapsto G_{n}(s, u) G_{n}(s, v) \frac{\left|D^{2} f(v-u, x-y)\right|}{|x-y|^{2 \bar{\rho}}}$ is the density of a finite measure on the set $[0, T] \times \mathcal{O} \times \mathcal{O} \times \mathbb{R}^{3} \times \mathbb{R}^{3}$, we can apply the Cauchy-Schwarz inequality with respect to this measure to obtain

$$
T_{n}^{4}(t, \mathcal{O}) \leq C\left(T_{n}^{4,1}(t, \mathcal{O}) T_{n}^{4,2}(t, \mathcal{O})\right)^{\frac{1}{2}}
$$


with

$$
\begin{aligned}
T_{n}^{4,1}(t, \mathcal{O})= & \int_{0}^{t} d s \int_{\mathcal{O}} d x \int_{\mathcal{O}} d y \int_{\mathbb{R}^{3}} d u \int_{\mathbb{R}^{3}} d v G_{n}(s, u) G_{n}(s, v) \\
& \times \frac{\left|D^{2} f(v-u, x-y)\right|}{|x-y|^{2 \bar{\rho}}} E\left(|Z(t-s, x-u)|^{q}\right), \\
T_{n}^{4,2}(t, \mathcal{O})= & \int_{0}^{t} d s \int_{\mathcal{O}} d x \int_{\mathcal{O}} d y \int_{\mathbb{R}^{3}} d u \int_{\mathbb{R}^{3}} d v G_{n}(s, u) G_{n}(s, v) \\
& \times \frac{\left|D^{2} f(v-u, x-y)\right|}{|x-y|^{2 \bar{\rho}}} E\left(|Z(t-s, x-v)|^{q}\right) .
\end{aligned}
$$

Using the new variables $\bar{x}=x-u, \bar{y}=y-u$, one can handle these terms as follows:

$$
\begin{aligned}
T_{n}^{4,1}(t, \mathcal{O}) \leq \int_{0}^{t} d s \int_{\mathcal{O}^{s k_{n}}} d \bar{x} \int_{\mathcal{O}^{s k_{n}}} d \bar{y} \int_{\mathbb{R}^{3}} d u \int_{\mathbb{R}^{3}} d v G_{n}(s, u) G_{n}(s, v) \\
\quad \times \frac{\left|D^{2} f(v-u, \bar{x}-\bar{y})\right|}{|\bar{x}-\bar{y}|^{2 \bar{\rho}}} E\left(|Z(t-s, \bar{x})|^{q}\right) \\
\leq C\left|\mathcal{O}^{2 T}\right| \sup _{n, x, y} \frac{\mu_{n}^{4}(x, y)}{|x-y|^{2 \bar{\rho}}} \int_{0}^{t} d s \int_{\mathcal{O}^{s k_{n}}} d \bar{x} E\left(|Z(t-s, \bar{x})|^{q}\right) \\
\leq C \int_{0}^{t} d s E\left(\|Z(s)\|_{L^{q}\left(\mathcal{O}^{\left.(t-s) k_{n}\right)}\right)}^{q}\right) .
\end{aligned}
$$

In the same way, with the change of variables $\bar{x}=x-v, \bar{y}=y-v$ we obtain a similar upper bound for the term $T_{n}^{4,2}(t, \mathcal{O})$. Consequently,

$$
T_{n}^{4}(t, \mathcal{O}) \leq C \int_{0}^{t} d s E\left(\|Z(s)\|_{L^{q}\left(\mathcal{O}^{(t-s)\left(1+\frac{1}{n}\right)}\right)}^{q}\right) .
$$

With (3.8)-(3.10), we obtain (3.5).

The inequality (3.6) is a consequence of (3.5). Indeed, take $\alpha=\rho+\frac{3}{q}$, which clearly satisfies the requirements of the statement. Hence, the proof of the lemma is complete.

With these ingredients, we can now proceed to the proof of Theorem 3.1. 
Proof of Theorem 3.1: Set $p=q / 2$ and fix $t \in] 0, T]$. Fix $m \in \mathbb{N}$ and let $\mathcal{O}_{m}=\left\{x \in \mathcal{O}: \overline{B_{1 / m}(x)} \subset \mathcal{O}\right\}$, where $\overline{B_{1 / m}(x)}$ is the closed Euclidean ball centered at $x$ with radius $1 / m$. Then $\left\{\mathcal{O}_{m}, m \in \mathbb{N}\right\}$ is an increasing sequence of open sets and $\cup_{m \in \mathbb{N}} \mathcal{O}_{m}=\mathcal{O}$. For $n \in \mathbb{N}$, let $k_{n}=1+\frac{1}{n}$. Observe that for $n \in \mathbb{N}$ such that $T / n<1 / m, \mathcal{O}_{m}^{(t-s) k_{n}} \subset \mathcal{O}^{t-s}$ if $0 \leq s \leq t \leq T$.

We start by showing that for such $m, n \in \mathbb{N}$,

$$
\begin{aligned}
E\left(\left\|v_{G_{n}, Z}^{t}\right\|_{L^{q}\left(\mathcal{O}_{m}\right)}^{q}\right) & \leq C \int_{0}^{t} d s J(t-s) E\left(\|Z(s)\|_{L^{q}\left(\mathcal{O}_{m}^{(t-s) k_{n}}\right)}^{q}\right) \\
& \leq C \int_{0}^{t} d s E\left(\|Z(s)\|_{L^{q}\left(\mathcal{O}_{m}^{(t-s) k_{n}}\right)}^{q}\right),
\end{aligned}
$$

where, for $0 \leq s<t \leq T$,

$$
J(t-s)=\sup _{\xi \in \mathbb{R}^{3}} \int_{\mathbb{R}^{3}} \mu(d \eta)|\mathcal{F} G(t-s)(\xi-\eta)|^{2} .
$$

Consider the (Walsh) stochastic integral $v_{G_{n}, Z}^{t}$ with $G_{n}$ given in (2.1). Set

$$
\mu_{n}(t, x)=\int_{0}^{t} d s \int_{\mathbb{R}^{3}} d y f(y)\left(G_{n}(s, x-\cdot) * \tilde{G}_{n}(s, x-\cdot)\right)(y) .
$$

By (2.5), (2.6), (2.7) and Assumption 2.4,

$$
\sup _{n, x, \bar{t} \leq T} \mu_{n}(\bar{t}, x) \leq \sup _{\bar{t} \leq T} \int_{0}^{\bar{t}} d s \int_{\mathbb{R}^{3}} \mu(d \xi)|\mathcal{F} G(s)(\xi)|^{2} \leq C .
$$

Burkholder's inequality yields

$$
\begin{aligned}
E\left(\left\|v_{G_{n}, Z}^{t}\right\|_{L^{q}\left(\mathcal{O}_{m}\right)}^{q}\right) \leq \int_{\mathcal{O}_{m}} d x( & \int_{0}^{t} d s \int_{\mathbb{R}^{3}} d u \int_{\mathbb{R}^{3}} d v f(u-v) \\
& \times G_{n}(t-s, x-u) G_{n}(t-s, x-v) \\
& \left.\times 1_{\mathcal{O}_{m}^{(t-s) k_{n}}}(u) Z(s, u) 1_{\mathcal{O}_{m}^{(t-s) k_{n}}}(v) Z(s, v)\right)^{p}
\end{aligned}
$$

(note that the presence of the indicator functions does not change the value of the integral, since $G_{n}(t-s, x-u)=0$ if $\left.u \notin \mathcal{O}_{m}^{(t-s) k_{n}}\right)$. Hölder's inequality yields

$$
\begin{array}{rl}
E\left(\left\|v_{G_{n}, Z}^{t}\right\|_{L^{q}\left(\mathcal{O}_{m}\right)}^{q}\right) \leq \int_{\mathcal{O}_{m}} & d x\left(\mu_{n}(T, x)\right)^{p-1} E\left(\int_{0}^{t} d s \int_{\mathbb{R}^{3}} d y f(y) \int_{\mathbb{R}^{3}} d z\right. \\
\times & G_{n}(s, x-z) G_{n}(s, x-y-z) \\
\times & \left.\left|Z_{m, n}(t-s, z)\right|^{p}\left|Z_{m, n}(t-s, z+y)\right|^{p}\right),
\end{array}
$$


where $Z_{m, n}(t-s, z)=1_{\mathcal{O}_{m}^{(t-s) k_{n}}}(z) Z(t-s, z)$.

Assume first that for any $s \in[0, T], Z_{m, n}(t-s) \in \mathcal{C}_{0}^{\infty}\left(\mathcal{O}_{m}^{(t-s) k_{n}}\right)$. In this case, $G_{n}(s, x-\cdot)\left|Z_{m, n}(s, \cdot)\right| \in \mathcal{C}_{0}^{\infty}\left(\mathcal{O}_{m}^{(t-s) k_{n}}\right)$, so the last integral is bounded by

$$
C E\left(\int_{\mathcal{O}_{m}} d x \int_{0}^{t} d s \int_{\mathbb{R}^{3}} \mu(d \eta)\left|\mathcal{F}\left[G_{n}(s, x-\cdot)\left|Z_{m, n}(t-s, \cdot)\right|^{p}\right](\eta)\right|^{2}\right) .
$$

We now apply the arguments of the proof of Lemma 1 of [7] (in particular (2.5) there), as follows. Since the Fourier transform of a product is the convolution of the Fourier transform of the factors, we have

$$
\begin{aligned}
\mathcal{F}\left[G_{n}(s, x-\cdot)\left|Z_{m, n}(t-s, \cdot)\right|^{p}\right](\eta) & \\
& =\int_{\mathbb{R}^{3}} d \xi^{\prime} e^{i x \cdot\left(\eta-\xi^{\prime}\right)} \mathcal{F} G_{n}(s, \cdot)\left(\eta-\xi^{\prime}\right) \mathcal{F}\left[\left|Z_{m, n}(t-s, \cdot)\right|^{p}\right]\left(\xi^{\prime}\right),
\end{aligned}
$$

where $x \cdot \xi^{\prime}$ denotes the Euclidean inner product in $\mathbb{R}^{3}$ of the vectors $x$ and $\xi^{\prime}$. By Plancherel's identity,

$$
\begin{aligned}
& \int_{\mathcal{O}_{m}} d x\left|\mathcal{F}\left[G_{n}(s, x-\cdot)\left|Z_{m, n}(t-s, \cdot)\right|^{p}\right](\eta)\right|^{2} \\
& \quad \leq \int_{\mathbb{R}^{3}} d x\left|\mathcal{F}\left[G_{n}(s, x-\cdot)\left|Z_{m, n}(t-s, \cdot)\right|^{p}\right](\eta)\right|^{2} \\
& \quad=\int_{\mathbb{R}^{3}} d \xi\left|\mathcal{F} G_{n}(s, \cdot)(\eta-\xi) \mathcal{F}\left(\left|Z_{m, n}(t-s, \cdot)\right|^{p}\right)(\xi)\right|^{2} .
\end{aligned}
$$

Consequently, (3.15) is bounded by

$$
\begin{aligned}
& C \int_{0}^{t} d s E\left(\int_{\mathbb{R}^{3}} d \xi\left|\mathcal{F}\left(\left|Z_{m, n}(t-s, \cdot)\right|^{p}\right)(\xi)\right|^{2}\right) \int_{\mathbb{R}^{3}} \mu(d \eta)\left|\mathcal{F} G_{n}(s)(\xi-\eta)\right|^{2} \\
& \leq C \int_{0}^{t} d s E\left(\int_{\mathbb{R}^{3}} d z\left|Z_{m, n}(t-s, z)\right|^{2 p}\right) \sup _{\xi \in \mathbb{R}^{3}} \int_{\mathbb{R}^{3}} \mu(d \eta)|\mathcal{F} G(s)(\xi-\eta)|^{2} \\
& \leq C \int_{0}^{t} d s E\left(\left\|Z_{m, n}(s)\right\|_{L^{q}\left(\mathbb{R}^{3}\right)}^{q}\right) \sup _{\xi \in \mathbb{R}^{3}} \int_{\mathbb{R}^{3}} \mu(d \eta)|\mathcal{F} G(t-s)(\xi-\eta)|^{2}
\end{aligned}
$$

Since supp $Z_{m, n}(s) \subset \mathcal{O}_{m}^{(t-s) k_{n}}$, we can replace $\|\cdot\|_{L^{q\left(\mathbb{R}^{3}\right)}}$ by $\|\cdot\|_{L^{q\left(\mathcal{O}_{m}^{(t-s) k_{n}}\right)}}$ in (3.17).

Without the smoothness and compact support restrictions on $Z_{m, n}(t-s)$, we can check, by regularizing $|Z| 1_{\mathcal{O}_{m}^{(t-s) k_{n}}}$ by means of convolution and using 
Fatou's Lemma, that the inequality

$$
\begin{aligned}
E & \left(\left\|v_{G_{n}, Z}^{t}\right\|_{L^{q}\left(\mathcal{O}_{m}\right)}^{q}\right) \\
& \leq C \int_{0}^{t} d s E\left(\|Z(s)\|_{L^{q}\left(\mathcal{O}_{m}^{(t-s) k_{n}}\right)}^{q}\right) \sup _{\xi \in \mathbb{R}^{3}} \int_{\mathbb{R}^{3}} \mu(d \eta)|\mathcal{F} G(t-s)(\xi-\eta)|^{2} \\
& =C \int_{0}^{t} d s J(t-s) E\left(\|Z(s)\|_{L^{q}\left(\mathcal{O}_{m}^{(t-s) k_{n}}\right)}^{q}\right)
\end{aligned}
$$

holds for any process $Z$ satisfying the assumptions of the Theorem. This proves (3.11).

Lemma 8 in [7] and (2.4) yield

$$
\sup _{0<s<t \leq T} J(t-s) \leq \int_{\mathbb{R}^{3}} \mu(d \eta)|\mathcal{F} G(t-s)(\eta)|^{2} \leq C .
$$

This establishes (3.12).

As in [7, Lemma 5], one checks that, for any fixed $m \in \mathbb{N}$,

$$
\begin{aligned}
\lim _{n \rightarrow \infty} \sup _{t \in[0, T]} E\left(\left\|v_{G_{n}, Z}^{t}-v_{G, Z}^{t}\right\|_{L^{2}\left(\mathcal{O}_{m}\right)}^{2}\right) \\
\leq \lim _{n \rightarrow \infty} \sup _{t \in[0, T]} E\left(\left\|v_{G_{n}, Z}^{t}-v_{G, Z}^{t}\right\|_{L^{2}\left(\mathcal{O}_{m}\right)}^{2}\right) \\
\quad=0 .
\end{aligned}
$$

By Fatou's Lemma, this yields

$$
E\left(\left\|v_{G, Z}^{t}\right\|_{L^{q}\left(\mathcal{O}_{m}\right)}^{q}\right) \leq \liminf _{k \rightarrow \infty} E\left(\left\|v_{G_{n_{k}}, Z}^{t}\right\|_{L^{q}\left(\mathcal{O}_{m}\right)}^{q}\right)
$$

for some subsequence $\left(n_{k}\right)_{k \geq 1}$.

Since $\mathcal{O}_{m}^{(t-s) k_{n}} \subset \mathcal{O}^{t-s}$, for any $n, m \in \mathbb{N}$ satisfying $\frac{T}{n}<\frac{1}{m}$, we deduce from (3.18) and (3.20) that

$$
E\left(\left\|v_{G, Z}^{t}\right\|_{L^{q}\left(\mathcal{O}_{m}\right)}^{q}\right) \leq C \int_{0}^{t} d s E\left(\|Z(s)\|_{L^{q}\left(\mathcal{O}^{t-s}\right)}^{q}\right) .
$$

Let $m \rightarrow \infty$. Using the monotone (increasing) convergence theorem, we obtain

$$
E\left(\left\|v_{G, Z}^{t}\right\|_{L^{q}(\mathcal{O})}^{q}\right) \leq C \int_{0}^{t} d s E\left(\|Z(s)\|_{L^{q}\left(\mathcal{O}^{t-s}\right)}^{q}\right) .
$$

Fix $\rho \in] 0, \tau_{q}(\beta, \delta) \wedge \gamma[$. By Fatou's Lemma and (3.6),

$$
\begin{aligned}
E\left(\left\|v_{G, Z}^{t}\right\|_{\rho, q, \mathcal{O}_{m}}^{q}\right) & \leq \liminf _{\ell \rightarrow \infty} E\left(\left\|v_{G_{n_{\ell}}, Z}^{t}\right\|_{\rho, q, \mathcal{O}_{m}}^{q}\right) \\
& \leq C \liminf _{\ell \rightarrow \infty} \int_{0}^{t} d s E\left(\|Z(s)\|_{W^{\rho, q}\left(\mathcal{O}_{m}^{(t-s)\left(1+\frac{1}{n_{\ell}}\right)}\right)}^{q}\right),
\end{aligned}
$$


for some subsequence $\left(n_{\ell}\right)_{\ell \geq 1}$. Since $\mathcal{O}_{m}^{(t-s)\left(1+\frac{1}{n_{\ell}}\right)} \subset \mathcal{O}^{t-s}$ for $\frac{T}{n_{l}}<\frac{1}{m}$, we obtain

$$
E\left(\left\|v_{G, Z}^{t}\right\|_{\rho, q, \mathcal{O}_{m}}^{q}\right) \leq C \int_{0}^{t} d s E\left(\|Z(s)\|_{W^{\rho, q}\left(\mathcal{O}^{t-s}\right)}^{q}\right) .
$$

Let $m \rightarrow \infty$. Using monotone convergence, we obtain

$$
E\left(\left\|v_{G, Z}^{t}\right\|_{\rho, q, \mathcal{O}}^{q}\right) \leq C \int_{0}^{t} d s E\left(\|Z(s)\|_{W^{\rho, q}\left(\mathcal{O}^{t-s}\right)}^{q}\right) .
$$

The inequalities (3.21) and (3.23) together establish (3.4). This completes the proof of the theorem.

Remark 3.3 Fix a bounded domain $\mathcal{O} \subset \mathbb{R}^{3}$ and assume that the stochastic process $Z$ in Theorem 3.1 is such that the right-hand side of (3.4) is finite for any $t \in] 0, T]$. By the Sobolev embedding theorem (see for instance [28, Theorem E.12 p.257]), Theorem 3.1 yields that, for each $t \in[0, T]$, a.s., $x \mapsto v_{G, Z}^{t}(x)$ is $a$-Hölder continuous, with $\left.a \in\right] 0,\left(\gamma \wedge \tau_{q}(\beta, \delta)\right)-\frac{3}{q}$ [. Indeed, for any bounded or unbounded domain $\mathcal{O} \subset \mathbb{R}^{d}, W^{\rho, q}(\mathcal{O}) \subset \mathcal{C}^{\beta}(\mathcal{O})$, for each $\beta<\rho-\frac{d}{q}$.

\subsection{Regularity in time of the stochastic integral}

This section is devoted to the analysis of the Hölder continuity in time of the stochastic integral process $\left\{v_{G, Z}^{t}(x), t \in[0, T]\right\}$, when $x$ is fixed. Throughout this section, $\mathcal{O}$ denotes a bounded domain in $\mathbb{R}^{3}$ and we shall make the following assumption on the integrand process $Z$ :

Assumption 3.4 For some fixed $q \in] 3, \infty[$ and $\gamma \in] 0,1[$,

$$
\sup _{t \in[0, T]} E\left(\|Z(t)\|_{W^{\gamma, q}\left(\mathcal{O}^{T-t}\right)}^{q}\right)<\infty
$$

Notice that Assumption 3.4 implies (3.3). Therefore, by Remark 3.3, it makes sense to fix the argument $x \in \mathcal{O}$ in the stochastic integral process. In addition, by the above mentioned Sobolev embedding, there is $C<\infty$ such that the Hölder norm $\|\cdot\|_{\mathcal{C}^{\rho}(\mathcal{O})}$ is bounded by a constant times the Sobolev norm $\|\cdot\|_{W^{\gamma, q}(\mathcal{O})}$, provided $\left.\rho \in\right] 0, \gamma-\frac{3}{q}[$. In particular, for any stochastic process $Z$ satisfying Assumption 3.4, one has

$$
\sup _{t \in[0, T]} E\left(|Z(t, x)-Z(t, y)|^{q}\right) \leq C|x-y|^{\rho q} .
$$


By Hölder's inequality, we deduce that for $\bar{q} \in] 0, q]$,

$$
\sup _{t \in[0, T]} E\left(|Z(t, x)-Z(t, y)|^{\bar{q}}\right) \leq C|x-y|^{\rho \bar{q}},
$$

for any $\left.\left.x, y \in \mathcal{O}^{T-t}, \rho \in\right] 0, \gamma-\frac{3}{q}[, \bar{q} \in] 0, q\right]$, with a positive constant $C$ not depending on $x, y$ or $\bar{q}$. Moreover,

$$
\sup _{t \in[0, T]} \sup _{x \in \mathcal{O}^{T-t}} E\left(|Z(t, x)|^{q}\right)<\infty \text {. }
$$

Theorem 3.5 Let $\tau(\beta, \delta)=\frac{2-\beta}{2} \wedge \frac{1+\delta}{2}$ and let $Z$ be a stochastic process satisfying Assumption 3.4 for a fixed $q \in] 3, \infty[$ and $\gamma \in] \frac{3}{q}, 1[$. Then the stochastic process $\left\{v_{G, Z}^{t}(x), t \in[0, T]\right\}\left(x \in \mathbb{R}^{3}\right)$ satisfies

$$
\sup _{x \in \mathcal{O}} E\left(\left|v_{G, Z}^{t}(x)-v_{G, Z}^{\bar{t}}(x)\right|^{\bar{q}}\right) \leq C|t-\bar{t}|^{\rho \bar{q}}
$$

for each $t, \bar{t} \in[0, T]$, any $\bar{q} \in] 2, q[$ and $\rho \in] 0,\left(\gamma-\frac{3}{q}\right) \wedge \tau(\beta, \delta)[$. Consequently, the process $\left(v_{G, Z}^{t}(x), t \in[0, T]\right)$ is a.s. a-Hölder continuous in $t$, for any $a \in$ ] $0,\left(\left(\gamma-\frac{3}{q}\right) \wedge \tau(\beta, \delta)\right)-\frac{3}{q}[$.

Proof: Let $\mathcal{O}_{m}$ be the open sets defined at the beginning of the proof of Theorem 3.1 and set $k_{n}=1+\frac{1}{n}$. Recall that $\mathcal{O}_{m}^{(t-s) k_{n}} \subset \mathcal{O}^{t-s}$ if $0 \leq s \leq t \leq T$ and $\frac{T}{n}<\frac{1}{m}$.

Set $p=\bar{q} / 2, p \in] 1, \infty[$ and fix $0 \leq t \leq \bar{t} \leq T$. The first part of the proof is devoted to showing that there is $C<\infty$ such that for all $m \in \mathbb{N}$,

$$
\sup _{n \geq m T} \sup _{x \in \mathcal{O}_{m}} E\left(\left|v_{G_{n}, Z}^{\bar{t}}(x)-v_{G_{n}, Z}^{t}(x)\right|^{2 p}\right) \leq C|t-\bar{t}|^{\tilde{\rho} p},
$$

with $\tilde{\rho} \in] 0,2\left(\left(\gamma-\frac{3}{2 p}\right) \wedge \tau(\beta, \delta)\right)\left[\right.$, where the $G_{n}, n \geq 1$, are defined in (2.1). Indeed, for $x \in \mathcal{O}_{m}$, consider the decomposition

$$
E\left(\left|v_{G_{n}, Z}^{\bar{t}}(x)-v_{G_{n}, Z}^{t}(x)\right|^{2 p}\right) \leq C\left(T_{1}^{n}(t, \bar{t}, x)+T_{2}^{n}(t, \bar{t}, x)\right),
$$

where

$$
\begin{gathered}
T_{1}^{n}(t, \bar{t}, x)=E\left(\left(\int_{t}^{\bar{t}} \int_{\mathbb{R}^{3}} G_{n}(\bar{t}-s, x-y) Z(s, y) M(d s, d y)\right)^{2 p}\right), \\
T_{2}^{n}(t, \bar{t}, x)=E\left(\left(\int_{0}^{t} \int_{\mathbb{R}^{3}}\left(G_{n}(\bar{t}-s, x-y)-G_{n}(t-s, x-y)\right)\right.\right. \\
\left.\times Z(s, y) M(d s, d y))^{2 p}\right) .
\end{gathered}
$$


Burkholder's inequality yields

$$
\begin{aligned}
T_{1}^{n}(t, \bar{t}, x) \leq & C E\left(\int_{t}^{\bar{t}} d s \int_{\mathbb{R}^{3}} d y \int_{\mathbb{R}^{3}} d z G_{n}(\bar{t}-s, x-y) G_{n}(\bar{t}-s, x-z)\right. \\
& \times f(y-z) Z(s, y) Z(s, z))^{p} \\
= & C E\left(\int_{0}^{\bar{t}-t} d s \int_{\mathbb{R}^{3}} d y \int_{\mathbb{R}^{3}} d z G_{n}(s, x-y) G_{n}(s, x-z)\right. \\
& \times f(y-z) Z(\bar{t}-s, y) Z(\bar{t}-s, z))^{p} .
\end{aligned}
$$

Set

$$
\mu^{n}(x, t, \bar{t})=\int_{0}^{\bar{t}-t} d s \int_{\mathbb{R}^{3}} d y \int_{\mathbb{R}^{3}} d z G_{n}(s, x-y) G_{n}(s, x-z) f(y-z) .
$$

By Assumption 2.4 and Lemma 2.2,

$$
\sup _{n \geq 1} \sup _{x \in \mathbb{R}^{3}} \mu^{n}(x, t, \bar{t}) \leq \int_{0}^{\bar{t}-t} d s \int_{\mathbb{R}^{3}} \frac{|\mathcal{F} G(s)(\xi)|^{2}}{|\xi|^{3-\beta}} d \xi \leq C|\bar{t}-t|^{3-\beta} .
$$

Hence, applying Hölder's inequality and using (3.25), we obtain

$$
\sup _{n \geq m T} \sup _{x \in \mathcal{O}_{m}} T_{1}^{n}(t, \bar{t}, x) \leq C|\bar{t}-t|^{p(3-\beta)}
$$

where $C$ does not depend on $m$.

We now study the contribution of $T_{2}^{n}(t, \bar{t}, x)$. We proceed first in a manner analogous to the proof of Theorem 3.1. After having applied Burkholder's inequality, changed variables (using (2.3)) and rearranged terms, we obtain

$$
T_{2}^{n}(t, \bar{t}, x) \leq C \sum_{i=1}^{4} E\left(\left|R^{i, n}(t, \bar{t}, x)\right|^{p}\right),
$$

where

$$
R^{i, n}(t, \bar{t}, x)=\int_{0}^{t} d s \int_{\mathbb{R}^{3}} d u \int_{\mathbb{R}^{3}} d v G_{n}(t-s, u) G_{n}(t-s, v) r_{i}(t, \bar{t}, s, x, u, v),
$$

and

$$
\begin{gathered}
r_{1}(t, \bar{t}, s, x, u, v)=f\left(\frac{\bar{t}-s}{t-s} v-u\right) \frac{\bar{t}-s}{t-s}\left(Z\left(s, x-\frac{\bar{t}-s}{t-s} u\right)-Z(s, x-u)\right) \\
\times\left(Z\left(s, x-\frac{\bar{t}-s}{t-s} v\right)-Z(s, x-v)\right),
\end{gathered}
$$




$$
\begin{aligned}
r_{2}(t, \bar{t}, s, x, u, v)= & \left.\left(\frac{\bar{t}-s}{t-s}\right)^{2} f\left(\frac{\bar{t}-s}{t-s}(v-u)\right)-\frac{\bar{t}-s}{t-s} f\left(\frac{\bar{t}-s}{t-s} v-u\right)\right) \\
& \times Z\left(s, x-\frac{\bar{t}-s}{t-s} u\right)\left(Z\left(s, x-\frac{\bar{t}-s}{t-s} v\right)-Z(s, x-v)\right), \\
r_{3}(t, \bar{t}, s, x, u, v)= & \left(\left(\frac{\bar{t}-s}{t-s}\right)^{2} f\left(\frac{\bar{t}-s}{t-s}(v-u)\right)-\frac{\bar{t}-s}{t-s} f\left(v-\frac{\bar{t}-s}{t-s} u\right)\right) \\
& \times Z(s, x-v)\left(Z\left(s, x-\frac{\bar{t}-s}{t-s} u\right)-Z(s, x-u)\right), \\
r_{4}(t, \bar{t}, s, x, u, v)= & \left(\left(\frac{\bar{t}-s}{t-s}\right)^{2} f\left(\frac{\bar{t}-s}{t-s}(v-u)\right)-\frac{\bar{t}-s}{t-s} f\left(\frac{\bar{t}-s}{t-s} v-u\right)\right. \\
& \left.-\frac{\bar{t}-s}{t-s} f\left(v-\frac{\bar{t}-s}{t-s} u\right)+f(v-u)\right) \\
& \times Z(s, x-u) Z(s, x-v) .
\end{aligned}
$$

Set

$$
\nu_{1}^{n}(s, t, \bar{t})=\int_{\mathbb{R}^{3}} d u \int_{\mathbb{R}^{3}} d v G_{n}(t-s, u) G_{n}(t-s, v) f\left(\frac{\bar{t}-s}{t-s} v-u\right) \frac{\bar{t}-s}{t-s} .
$$

By Lemma 6.3, we can apply Hölder's inequality and then Schwarz's inequality to obtain

$$
\begin{aligned}
& E\left(\left|R^{1, n}(t, \bar{t}, x)\right|^{p}\right) \leq\left(\sup _{n \geq 1} \sup _{0 \leq s \leq t \leq \bar{t} \leq T} \nu_{1}^{n}(s, t, \bar{t})\right)^{p-1} \\
& \times \int_{0}^{t} d s \int_{\mathbb{R}^{3}} d u \int_{\mathbb{R}^{3}} d v G_{n}(t-s, u) G_{n}(t-s, v) f\left(\frac{\bar{t}-s}{t-s} v-u\right) \\
& \times \frac{\bar{t}-s}{t-s}\left(E\left(\left|Z\left(s, x-\frac{\bar{t}-s}{t-s} u\right)-Z(s, x-u)\right|^{2 p}\right)\right)^{1 / 2} \\
& \quad \times\left(E\left(\left|Z\left(s, x-\frac{\bar{t}-s}{t-s} v\right)-Z(s, x-v)\right|^{2 p}\right)\right)^{1 / 2} .
\end{aligned}
$$


By (3.24), for $\rho \in] 0, \gamma-\frac{3}{q}[$, the product of the last two factors is bounded by

$$
\frac{\bar{t}-s}{t-s}\left(\frac{\bar{t}-t}{t-s}\right)^{2 p \rho}|u|^{\rho p}|v|^{\rho p}
$$

Since for any $t \in(0, T]$, supp $G_{n}(t, \cdot) \subset B_{t\left(1+\frac{1}{n}\right)}(0),\left(|u||v| /|t-s|^{2}\right)^{\rho p}$ in the last integral is bounded by a finite constant. Therefore, by Lemma 6.3,

$$
\begin{aligned}
\sup _{n \geq m T} \sup _{x \in \mathcal{O}_{m}} E\left(\left|R^{1, n}(t, \bar{t}, x)\right|^{p}\right) & \leq C|t-\bar{t}|^{2 p \rho} \sup _{n \geq 1} \sup _{0 \leq s \leq t \leq \bar{t} \leq T}\left(\nu_{1}^{n}(t, \bar{t})\right)^{p} \\
& \leq C|t-\bar{t}|^{2 p \rho}
\end{aligned}
$$

with $\rho \in] 0, \gamma-\frac{3}{q}[$ and $C$ does not depend on $m$.

Taking into account the result proved in Lemma 6.4 and using the quantity $\nu_{2}^{n}(t, \bar{t})$ defined in that lemma, we apply first Hölder's inequality, then Schwarz's inequality, to obtain

$$
\begin{aligned}
E\left(\left|R^{2, n}(t, \bar{t}, x)\right|^{p}\right) \leq & \left(\nu_{2}^{n}(t, \bar{t})\right)^{p-1} \int_{0}^{t} d s \int_{\mathbb{R}^{3}} d u \int_{\mathbb{R}^{3}} d v G_{n}(t-s, u) G_{n}(t-s, v) \\
& \times\left|\left(\frac{\bar{t}-s}{t-s}\right)^{2} f\left(\frac{\bar{t}-s}{t-s}(v-u)\right)-\frac{\bar{t}-s}{t-s} f\left(\frac{\bar{t}-s}{t-s} v-u\right)\right| \\
& \times\left(E\left(\left|Z\left(s, x-\frac{\bar{t}-s}{t-s} u\right)\right|^{2 p}\right)\right)^{1 / 2} \\
& \times\left(E\left(\left|Z\left(s, x-\frac{\bar{t}-s}{t-s} v\right)-Z(s, x-v)\right|^{2 p}\right)\right)^{1 / 2} .
\end{aligned}
$$

Then, by Lemma 6.4, (3.24) and (3.25),

$$
\sup _{n \geq m T} \sup _{x \in \mathcal{O}_{m}} E\left(\left|R^{2, n}(t, \bar{t}, x)\right|^{p}\right) \leq C|t-\bar{t}|^{p(\rho+\alpha)}
$$

for any $\alpha \in] 0,1[$ with $\alpha+\beta \in] 0,2[, \rho \in] 0, \gamma-\frac{3}{q}[$, and $C$ does not depend on $m$. The same result holds for the term $R^{3, n}(t, \bar{t}, x)$. 
Using Lemma 6.5 and the quantity $\nu_{3}^{n}(t, \bar{t})$ defined in that lemma, we can apply Hölder's inequality to obtain

$$
\begin{aligned}
E\left(\left|R^{4, n}(t, \bar{t}, x)\right|^{p}\right) \leq & \left(\nu_{3}^{n}(t, \bar{t})\right)^{p-1} \int_{0}^{t} d s \int_{\mathbb{R}^{3}} d u \int_{\mathbb{R}^{3}} d v G_{n}(t-s, u) G_{n}(t-s, v) \\
& \times\left|\Delta^{2} f(s, t, \bar{t}, u, v)\right| E\left(|Z(s, x-u)|^{p}|Z(s, x-v)|^{p}\right),
\end{aligned}
$$

where $\Delta^{2} f(s, t, \bar{t}, u, v)$ is defined in (6.15).

Finally, by Schwarz's inequality, property (3.25) and (6.16), we reach

$$
\begin{aligned}
\sup _{n \geq m T} \sup _{x \in \mathcal{O}_{m}} E\left(\left|R^{4, n}(t, \bar{t}, x)\right|^{p}\right) & \leq C \sup _{n \geq 1}\left(\nu_{3}^{n}(t, \bar{t})\right)^{p} \sup _{t \in[0, T]} \sup _{x \in \mathcal{O}^{T-t}} E\left(|Z(t, x)|^{2 p}\right) \\
& \leq C|t-\bar{t}|^{\alpha p}
\end{aligned}
$$

with $\alpha \in] 0,(2-\beta) \wedge(1+\delta)[$ and $C$ does not depend on $m$. Hence, (3.28)(3.31) establish (3.27).

The second part of the proof consists in deducing (3.26) from (3.27). To this end, we first prove that for any fixed $m \in \mathbb{N}$ and $t \in[0, T],\left(v_{G_{n}, Z}^{t}, n \geq\right.$ $m T)$ is a sequence of bounded and equicontinuous functions defined on $\mathcal{O}_{m}$ with values in $L^{\bar{q}}(\Omega)$, for any $\bar{q} \in[1, q]$. Indeed, from (3.18) and (3.6) together with Assumption 3.4 and the inclusion $\mathcal{O}_{m}^{(t-s) k_{n}} \subset \mathcal{O}^{t-s}$, we see that

$$
\sup _{m \in \mathbb{N}} \sup _{n \geq m T} \sup _{t \in[0, T]} E\left(\left\|v_{G_{n}, Z}^{t}\right\|_{W^{\rho, q}\left(\mathcal{O}_{m}\right)}^{q}\right)<\infty
$$

for any $\rho \in] 0, \rho_{0}\left[\right.$, with $\rho_{0}=\gamma \wedge\left(\tau(\beta, \delta)-\frac{3}{q}\right)$. Therefore, the Sobolev embedding yields

$$
\sup _{m \in \mathbb{N}} \sup _{n \geq m T} \sup _{t \in[0, T]} E\left(\left\|v_{G_{n}, Z}^{t}\right\|_{\mathcal{C}^{\tilde{\rho}}\left(\mathcal{O}_{m}\right)}^{q}\right)<\infty
$$

for any $\tilde{\rho} \in] 0, \rho_{0}-\frac{3}{q}\left[\right.$. Consequently, for any $x, y \in \mathcal{O}_{m}$,

$$
\sup _{n \geq m T} E\left(\left|v_{G_{n}, Z}^{t}(x)-v_{G_{n}, Z}^{t}(y)\right|^{\bar{q}}\right) \leq C|x-y|^{\tilde{\rho} \bar{q}}
$$

for every $\bar{q} \in[1, q]$, where $C$ does not depend on $m$. This establishes the desired boundedness and equicontinuity properties.

We now establish a uniform convergence result. The convergence (3.20) implies the existence of a subsequence $\left(n_{k}, k \geq 1\right)$ and a Lebesgue-measurable subset $N \subset \mathcal{O}_{m}$ with null Lebesgue measure such that for any $x \in \mathcal{O}_{m} \backslash N$,

$$
\lim _{k \rightarrow \infty} E\left(\left|v_{G_{n_{k}}, Z}^{t}(x)-v_{G, Z}^{t}(x)\right|^{2}\right)=0 .
$$


The property (3.33) yields $\sup _{n \geq m T} E\left(\left|v_{G_{n}, Z}^{t}(x)\right|^{q}\right)<\infty$. Hence, by uniform integrability, for any $x \in \mathcal{O}_{m} \backslash N$ and for any $\bar{q} \in[1, q[$,

$$
\lim _{k \rightarrow \infty} E\left(\left|v_{G_{n_{k}, Z}}^{t}(x)-v_{G, Z}^{t}(x)\right|^{\bar{q}}\right)=0 .
$$

Thus, we have proved that the bounded and equicontinuous sequence $\left(v_{G_{n_{b}}, Z}^{t}, k \geq 1\right)$ of functions converges pointwise in $L^{\bar{q}}(\Omega)$, on a dense subset $\mathcal{O}_{m} \backslash N$ of $\mathcal{O}_{m}$. Therefore, since $v_{G, Z}^{t}$ is $L^{\bar{q}}(\Omega)$-continuous (see Remark 3.3), we have

$$
\lim _{k \rightarrow \infty} \sup _{x \in \mathcal{O}_{m}} E\left(\left|v_{G_{n_{k}}, Z}^{t}(x)-v_{G, Z}^{t}(x)\right|^{\bar{q}}\right)=0 .
$$

(see for instance $[9$, Ch. VII] $)$, that is, $\left(v_{G_{n_{k}}, Z}^{t}, k \in \mathbb{N}\right) \subset C\left(\mathcal{O}_{m}, L^{\bar{q}}(\Omega)\right)$ converges uniformly to $v_{G, Z}^{t}$.

Clearly, for any $m \in \mathbb{N}$ and $t, \bar{t} \in[0, T]$,

$$
\sup _{x \in \mathcal{O}_{m}} E\left(\left|v_{G, Z}^{t}(x)-v_{G, Z}^{\bar{t}}(x)\right|^{\bar{q}}\right) \leq C \sum_{i=1}^{3} A_{i}(t, \bar{t}),
$$

where $C$ does not depend on $m$ and

$$
\begin{aligned}
& A_{1}(t, \bar{t})=\sup _{x \in \mathcal{O}_{m}} E\left(\left|v_{G, Z}^{t}(x)-v_{G_{n_{k}}, Z}^{t}(x)\right|^{\bar{q}}\right), \\
& A_{2}(t, \bar{t})=\sup _{k \geq 1} \sup _{x \in \mathcal{O}_{m}} E\left(\left|v_{G_{n_{k}}, Z}^{t}(x)-v_{G_{n_{k}}, Z}^{\bar{t}}(x)\right|^{\bar{q}}\right), \\
& A_{3}(t, \bar{t})=\sup _{x \in \mathcal{O}_{m}} E\left(\left|v_{G_{n_{k}}, Z}^{\bar{t}}(x)-v_{G, Z}^{\bar{t}}(x)\right|^{\bar{q}}\right) .
\end{aligned}
$$

By (3.36), (3.35) and (3.27), we obtain

$$
\sup _{x \in \mathcal{O}_{m}} E\left(\left|v_{G, Z}^{t}(x)-v_{G, Z}^{\bar{t}}(x)\right|^{\bar{q}}\right) \leq C|t-\bar{t}|^{\rho \bar{q}}
$$

for any $\rho \in] 0,\left(\gamma-\frac{3}{q}\right) \wedge \tau(\beta, \delta)\left[\right.$. Since $C$ does not depend on $m, \mathcal{O}_{m}$ can de replaced by $\mathcal{O}$ in the inequality above and this establishes (3.26).

\section{Path properties of the solution of the sto- chastic wave equation}

This section is devoted to studying the properties of the sample paths of the stochastic wave equation in spatial dimension three. More precisely, consider the s.p.d.e.

$$
\left.\left.\left(\frac{\partial^{2}}{\partial t^{2}}-\Delta\right) u(t, x)=\sigma(u(t, x)) \dot{F}(t, x)+b(u(t, x)), t \in\right] 0, T\right], x \in \mathbb{R}^{3},
$$


with initial conditions $u(0, x)=v_{0}(x), \frac{\partial}{\partial t} u(0, x)=\tilde{v}_{0}(x)$. We are interested in the solution $u(t, x)$ of this equation for $(t, x) \in] 0, T] \times D$, where $D$ denotes a bounded domain of $\mathbb{R}^{3}$ included in the ball $B_{m_{0}}(0)$, for some $m_{0}>0$.

For any $a \geq 1$, set

$$
K_{a}^{D}(t)=\left\{y \in \mathbb{R}^{3}: d(y, D) \leq a(T-t)\right\}, t \in[0, T],
$$

where $d$ denotes the Euclidean distance. Notice that $t \mapsto K_{a}^{D}(t)$ is decreasing.

\subsection{Existence, uniqueness and moments}

Recall that the solution $w$ of the homogeneous wave equation with the same initial conditions as $u$ is $w(t)=\frac{d}{d t} G(t) * v_{0}+G(t) * \tilde{v}_{0}$. We are mainly interested in the solution of (4.1) for $(t, x) \in[0, T] \times D$, though we will need to construct the solution in a slightly larger set that contains the "past light cone" of $\{T\} \times D$. Therefore, we term a solution of the s.p.d.e. (4.1) "in D" a stochastic process $\left(u(t) 1_{K_{a}^{D}(t)}, t \in[0, T]\right)$ with values in $L^{2}\left(\mathbb{R}^{3}\right)$, satisfying

$$
\begin{aligned}
& u(t, \cdot) 1_{K_{a}^{D}(t)}(\cdot)=1_{K_{a}^{D}(t)}(\cdot)\left(\frac{d}{d t} G(t) * v_{0}+G(t) * \tilde{v}_{0}\right)(\cdot) \\
& \quad+1_{K_{a}^{D}(t)}(\cdot) \int_{0}^{t} \int_{\mathbb{R}^{3}} G(t-s, \cdot-y) \sigma(u(s, y)) 1_{K_{a}^{D}(s)}(y) M(d s, d y) \\
& \quad+1_{K_{a}^{D}(t)}(\cdot) \int_{0}^{t} d s G(t-s) *\left(b(u(s, \cdot)) 1_{K_{a}^{D}(s)}(\cdot)\right)
\end{aligned}
$$

a.s., for any $t \in[0, T]$. The integrands in (4.2) have compact support. Further, the support of the measure $G(t-s)$ is the boundary of the ball $B_{t-s}(0)$. Therefore, if $x \in K_{a}^{D}(t)$ and $x-y \in \partial B_{t-s}(0)$, then $d(y, D) \leq$ $d(y, x)+d(x, D) \leq t-s+a(T-t) \leq a(T-s)$. Consequently, $y \in K_{a}^{D}(s)$. Therefore, $\left(K_{a}^{D}(t)\right)^{t-s} \subset K_{a}^{D}(s)$ and so (4.2) is coherent.

The stochastic integral in (4.2) is to be considered in the sense of [7, Section 2]. We note that we have introduced the indicator functions $1_{K_{a}^{D}(s)}(y)$ in order to use this particular stochastic integral, which requires that the integrands be square-integrable over all of $\mathbb{R}^{3}$.

Concerning the pathwise integral, we now give some details. Let $(Z(s), s \in$ $[0, T])$ be a stochastic process taking its values in $L^{2}\left(\mathbb{R}^{d}\right)$ satisfying

$$
\sup _{t \in[0, T]} E\left(\|Z(t)\|_{L^{2}\left(\mathbb{R}^{d}\right)}^{2}\right)<\infty .
$$

Let $G:[0, T] \rightarrow \mathcal{S}^{\prime}\left(\mathbb{R}^{d}\right)$ be such that for any $s \in[0, T], \mathcal{F} G(s)$ is a function and

$$
\int_{0}^{T} d s \sup _{\xi \in \mathbb{R}^{d}}|\mathcal{F} G(s)(\xi)|^{2}<\infty
$$


(notice that the fundamental solution of the wave equation satisfies this condition). Then for any $t \in[0, T]$,

$$
J_{G, Z}^{t}(x):=\int_{0}^{t} d s(G(s) * Z(s))(x)
$$

defines a function in $L^{2}\left(\mathbb{R}^{d}\right)$, a.s. . Moreover,

$$
\left\|J_{G, Z}^{t}\right\|_{L^{2}\left(\mathbb{R}^{d}\right)}^{2} \leq t \int_{0}^{t} d s \sup _{\xi \in \mathbb{R}^{d}}|\mathcal{F} G(s)(\xi)|^{2}\|Z(s)\|_{L^{2}\left(\mathbb{R}^{d}\right)}^{2},
$$

hence

$$
E\left(\left\|J_{G, Z}^{t}\right\|_{L^{2}\left(\mathbb{R}^{d}\right)}^{2}\right) \leq t \int_{0}^{t} d s \sup _{\xi \in \mathbb{R}^{d}}|\mathcal{F} G(s)(\xi)|^{2} E\left(\|Z(s)\|_{L^{2}\left(\mathbb{R}^{d}\right)}^{2}\right) .
$$

For higher moments, assume that $d=3,(Z(s), s \in[0, T])$ takes values in $L^{q}\left(\mathbb{R}^{3}\right)$ for some $q \in[1, \infty[$ and that $G(t)$ is the fundamental solution of the wave equation in $\mathbb{R}^{3}$. Then, Fubini's theorem and the facts that $G \geq 0$ and $\operatorname{supp} G(s)=\partial B_{s}(0)$ imply that

$$
\|G(s) * Z(s)\|_{L^{q}(D)}^{q} \leq\left(\int_{\mathbb{R}^{3}} G(s, d y)\right)^{q}\|Z(s)\|_{L^{q}\left(D^{s}\right)}^{q}=s^{q}\|Z(s)\|_{L^{q}\left(D^{s}\right)}^{q} .
$$

From Hölder's inequality, it follows that

$$
E\left(\left\|J_{G, Z}^{t}\right\|_{L^{q}(D)}^{q}\right) \leq C \int_{0}^{t} d s\left(\int_{\mathbb{R}^{3}} G(s, d y)\right)^{q} E\left(\|Z(s)\|_{L^{q}\left(D^{s}\right)}^{q}\right) .
$$

The next theorem gives existence and uniqueness of the solution of equation (4.2) and states some of its properties. Notice that in comparison with Theorems 9 and 13 of [7], we allow a non vanishing coefficient $b$ and avoid introducing a weight function.

Theorem 4.1 Let $\sigma, b: \mathbb{R} \rightarrow \mathbb{R}$ be Lipschitz continuous functions, $v_{0}$, $\tilde{v}_{0}$ be real-valued functions such that $v_{0}$ is of class $C^{2}$ and $\tilde{v}_{0} \in L_{l o c}^{q}\left(\mathbb{R}^{3}\right)$, for some $q \in\left[2, \infty\left[\right.\right.$. Then there exists a unique process $\left(u(t) 1_{K_{a}^{D}(t)}, t \in[0, T]\right)$ with values in $L^{2}\left(\mathbb{R}^{3}\right)$ satisfying equation (4.2).

Moreover, for any $t \in[0, T]$,

$$
E\left(\|u(t)\|_{L^{q}\left(K_{a}^{D}(t)\right)}^{q}\right) \leq C\left(1+I_{a}(t)\right)
$$

with

$$
I_{a}(t):=\left\|v_{0}\right\|_{L^{q}\left(\left(K_{a}^{D}(t)\right)^{t}\right)}^{q}+\left\|\Delta v_{0}\right\|_{L^{q}\left(\left(K_{a}^{D}(t)\right)^{t}\right)}^{q}+\left\|\tilde{v}_{0}\right\|_{L^{q}\left(\left(K_{a}^{D}(t)\right)^{t}\right)}^{q} .
$$


As a consequence,

$$
\sup _{t \in[0, T]} E\left(\|u(t)\|_{L^{q}\left(K^{D}(t)\right)}^{q}\right) \leq C(1+J)
$$

where $K^{D}(t)=K_{1}^{D}(t)$ and

$$
J=\left\|v_{0}\right\|_{L^{q}\left(D^{T}\right)}^{q}+\left\|\Delta v_{0}\right\|_{L^{q}\left(D^{T}\right)}^{q}+\left\|\tilde{v}_{0}\right\|_{L^{q}\left(D^{T}\right)}^{q} .
$$

Before proving this theorem, we state some results concerning the initial conditions. First, we notice that under the hypotheses of the previous theorem, $J$ and $I_{a}(t)$ are finite for any $a \geq 1$ and $t \in[0, T]$.

For $v_{0} \in L^{2}\left(\mathbb{R}^{3}\right)$, it is well-known that

$$
\frac{d}{d t} G(t) * v_{0}=\frac{1}{t}\left(v_{0} * G(t)\right)+\frac{1}{4 \pi} \int_{|y|<1}\left(\Delta v_{0}\right)(\cdot+t y) d y,
$$

(see for instance [29]).

Lemma 4.2 Let $v_{0}, \tilde{v}_{0}$ be real-valued functions and $\mathcal{O}$ be a bounded domain of $\mathbb{R}^{3}$.

(a) Suppose that $v_{0} \in C^{2}\left(\mathcal{O}^{T}\right)$. Then for any $t \in[0, T]$,

$$
\left\|\frac{d}{d t} G(t) * v_{0}\right\|_{L^{q}(\mathcal{O})}^{q} \leq C\left(\left\|v_{0}\right\|_{L^{q}\left(\mathcal{O}^{t}\right)}^{q}+\left\|\Delta v_{0}\right\|_{L^{q}\left(\mathcal{O}^{t}\right)}^{q}\right),
$$

for any $q \in[2, \infty[$.

(b) Assume that $\tilde{v}_{0} \in L^{q}\left(\mathcal{O}^{T}\right)$ for some $q \in[2, \infty[$. Then for any $t \in[0, T]$,

$$
\left\|G(t) * \tilde{v}_{0}\right\|_{L^{q}(\mathcal{O})} \leq\left\|\tilde{v}_{0}\right\|_{L^{q}\left(\mathcal{O}^{t}\right)}
$$

Proof: By (4.11),

$$
\left\|\frac{d}{d t} G(t) * v_{0}\right\|_{L^{q}(\mathcal{O})}^{q} \leq C(A(t)+B(t)),
$$

where

$$
A(t)=\left\|\frac{1}{t}\left(v_{0} * G(t)\right)\right\|_{L^{q}(\mathcal{O})}^{q}, \quad B(t)=\left\|\frac{1}{t^{3}}\left(\Delta v_{0} * 1_{B_{t}(0)}\right)\right\|_{L^{q}(\mathcal{O})}^{q} .
$$


Inequality (4.6) yields

$$
A(t) \leq \frac{1}{t^{q}}\left(\int_{\mathbb{R}^{3}} G(t, d u)\right)^{q}\left\|v_{0}\right\|_{L^{q}\left(\mathcal{O}^{t}\right)}^{q}=\left\|v_{0}\right\|_{L^{q}\left(\mathcal{O}^{t}\right)}^{q} .
$$

Similarly,

$$
B(t) \leq C\left\|\Delta v_{0}\right\|_{L^{q}\left(\mathcal{O}^{t}\right)}^{q}, \quad \text { and } \quad\left\|G(t) * \tilde{v}_{0}\right\|_{L^{q}(\mathcal{O})}^{q} \leq\left\|\tilde{v}_{0}\right\|_{L^{q}\left(\mathcal{O}^{t}\right)}^{q} .
$$

This proves the lemma.

Proof of Theorem 4.1. Consider the Picard iteration scheme given by

$$
u^{0}(t, \cdot)=\frac{d}{d t} G(t) * v_{0}+G(t) * \tilde{v}_{0},
$$

and, for $n \geq 1$,

$$
\begin{aligned}
& u^{n}(t, \cdot) 1_{K_{a}^{D}(t)}(\cdot)=1_{K_{a}^{D}(t)}(\cdot)\left(u^{0}(t, \cdot)\right.+\int_{0}^{t} \int_{\mathbb{R}^{3}} G(t-s, \cdot-y) \sigma\left(u^{n-1}(s, y)\right) \\
&\left.\times 1_{K_{a}^{D}(s)}(y) M(d s, d y)\right) \\
&+1_{K_{a}^{D}(t)}(\cdot) \int_{0}^{t} d s G(t-s) *\left(b\left(u^{n-1}(s, \cdot)\right) 1_{K_{a}^{D}(s)}(\cdot)\right)(\cdot) .
\end{aligned}
$$

For $n \geq 0$, set $v^{n}(t, \cdot)=u^{n}(t, \cdot) 1_{K_{a}^{D}(t)}(\cdot)$. Lemma 4.2 with $\mathcal{O}:=K_{a}^{D}(t)$, along with the inequalities (3.21) and (4.7) applied to $Z(s):=g\left(u^{n-1}(s, \cdot)\right) 1_{K_{a}^{D}(s)}(\cdot)$ with $g \equiv \sigma$ and $g \equiv b$, respectively, and the Lipschitz properties of $\sigma$ and $b$, tell us that for any $n \geq 1$,

$$
\begin{aligned}
E\left(\left\|v^{n}(t)\right\|_{L^{q}\left(K_{a}^{D}(t)\right)}^{q}\right) & \leq C I_{a}(t)+C_{1} \int_{0}^{t} d s E\left(\int_{\mathbb{R}^{3}} d x 1_{K_{a}^{D}(s)}(x)\left(1+\left|u^{n-1}(s, x)\right|^{q}\right)\right) \\
& \leq C I_{a}(t)+C_{1} \int_{0}^{t} d s\left(1+E\left(\left\|v^{n-1}(s)\right\|_{L^{q}\left(K_{a}^{D}(s)\right)}^{q}\right)\right) .
\end{aligned}
$$

Notice that $v^{0}(t, \cdot) 1_{K_{a}^{D}(t)}(\cdot)=u^{0}(t, \cdot) 1_{K_{a}^{D}(t)}(\cdot)$ and by Lemma 4.2,

$$
E\left(\left\|v^{0}(t)\right\|_{L^{q}\left(K_{a}^{D}(t)\right)}^{q}\right)=\left(\left\|u^{0}(t)\right\|_{L^{q}\left(K_{a}^{D}(t)\right)}^{q}\right) \leq C I_{a}(t)<\infty .
$$

Thus, we obtain existence, uniqueness and (4.8) using arguments similar to those in the proof of Theorem 9 in [7], based on Gronwall's lemma.

A priori, the solution should be written $\left(u_{a}(t) 1_{K_{a}^{D}(t)}, t \in[0, T]\right)$. However, $u_{a}(t)$ does not depend on $a$. Indeed, for $1 \leq a \leq b$, both $\left(u_{a}(t) 1_{K_{a}^{D}(t)}, t \in\right.$ 
$[0, T])$ and $\left(\left(u_{b}(t) 1_{K_{b}^{D}(t)}\right) 1_{K_{a}^{D}(t)}, t \in[0, T]\right)$ satisfy (4.2), so uniqueness for $a$ implies that $u_{a}(t) 1_{K_{a}^{D}(t)}=u_{b}(t) 1_{K_{a}^{D}(t)}$.

We now prove (4.10). For any $m \geq 1$, set $D_{m}=\left\{x \in D: \overline{B_{1 / m}(x)} \subset D\right\}$ and $a_{m}:=1+\frac{1}{m^{2}}$. We notice that for any $m \geq T-t$, and $t \in[0, T]$, the set $\left(K_{a_{m}}^{D_{m}}(t)\right)^{t}$ is included in $D^{T}$. Consequently, $I_{a_{m}}(t) \leq J$. Moreover, the sequence of sets $K_{a_{m}}^{D_{m}}(t), m \geq 2(T-t)$, is increasing and $\cup_{m} K_{a_{m}}^{D_{m}}(t)=K^{D}(t)$. Hence, (4.10) follows from (4.8) by letting $m$ tend to $\infty$.

\subsection{Regularity in the space variable}

The solution of equation (4.2) can be approximated by a sequence of solutions of similar equations obtained by regularising the fundamental solution $G$ in the term involving a stochastic integral. More precisely, let $G_{n}(t, x), n \geq$ 1 , be as in (2.1), fix a bounded domain $D \subset \mathbb{R}^{3}$ satisfying the conditions described at the beginning of the section and set, for any $a>1$ and $n$ with $1+\frac{1}{n}<a$,

$$
\begin{aligned}
& u_{n}(t, x) 1_{K_{a}^{D}(t)}(x)=1_{K_{a}^{D}(t)}(x)\left(\frac{d}{d t} G(t) * v_{0}+G(t) * \tilde{v}_{0}\right)(x) \\
& \quad+1_{K_{a}^{D}(t)}(x) \int_{0}^{t} \int_{\mathbb{R}^{3}} G_{n}(t-s, x-y) \sigma\left(u_{n}(s, y)\right) 1_{K_{a}^{D}(s)}(y) M(d s, d y) \\
& \quad+1_{K_{a}^{D}(t)}(x) \int_{0}^{t} d s\left(G(t-s) *\left(b\left(u_{n}(s, \cdot)\right) 1_{K_{a}^{D}(s)}(\cdot)\right)\right)(x) .
\end{aligned}
$$

Since $G_{n}$ is smooth, the stochastic integral in (4.12) is considered in Walsh's sense [32]. In particular, we could remove the $1_{K_{a}^{D}(t)}(x)$ and $1_{K_{a}^{D}(s)}(y)$ from (4.12).

Proposition 4.3 Let $\sigma, b, v_{0}$ and $\tilde{v}_{0}$ be as in Theorem 4.1. Then there exists a unique process $\left\{u_{n}(t, \cdot) 1_{K_{a}^{D}(t)}(\cdot), t \in[0, T]\right\}$ solution of (4.12), and this process is such that for $q \in[2, \infty[$,

$$
\sup _{n \geq(a-1)^{-1}} \sup _{t \in[0, T]} E\left(\left\|u_{n}(t)\right\|_{L^{q}\left(K_{a}^{D}(t)\right)}^{q}\right)<\infty .
$$

Moreover

$$
\lim _{n \rightarrow \infty} \sup _{t \in[0, T]} E\left(\left\|u_{n}(t)-u(t)\right\|_{L^{q}\left(K_{a}^{D}(t)\right)}^{q}\right)=0 .
$$

Proof: The proof of existence, uniqueness and (4.13) can be obtained using arguments similar to those applied in Theorem 4.1, taking into account (2.6). 
To prove (4.14), we apply the $L^{p}$-estimates of the stochastic and the pathwise integrals given in (3.21) and (4.7), respectively (with $G$ replaced by $\left.G_{n}\right)$. Let $n \geq 1$ be such that $1+\frac{1}{n} \leq a$; then

$$
\left(K_{a}^{D}(t)\right)^{(t-s)(1+1 / n)} \subset K_{a}^{D}(s),
$$

for $0 \leq s \leq t \leq T$ and we obtain

$$
\begin{aligned}
& E\left(\left\|u_{n}(t)-u(t)\right\|_{L^{q}\left(K_{a}^{D}(t)\right)}^{q}\right) \\
& \quad \leq C \int_{0}^{t} d s E\left(\left\|\left(u_{n}(s)-u(s)\right)\right\|_{L^{q}\left(K_{a}^{D}(s)\right)}^{q}\right)+M_{n}(t),
\end{aligned}
$$

where

$$
\begin{aligned}
M_{n}(t)= & E\left(\| \int_{0}^{t} \int_{\mathbb{R}^{3}}\left(G_{n}(t-s, \cdot-y)-G(t-s, \cdot-y)\right)\right. \\
& \left.\quad \times \sigma(u(s, y)) 1_{K_{a}^{D}(s)}(y) M(d s, d y) \|_{L^{q}\left(K_{a}^{D}(t)\right)}^{q}\right) . \\
= & E\left(\left\|v_{G_{n}, Z}-v_{G, Z}\right\|_{L^{q}\left(K_{a}^{D}(t)\right)}^{q}\right),
\end{aligned}
$$

and $Z(s, y)=\sigma(u(s, y)) 1_{K_{a}^{D}(s)}(y)$.

We shall show that

$$
\lim _{n \rightarrow \infty} M_{n}(t)=0 .
$$

Since (4.14) follows from this property, (4.16) and Gronwall's lemma, the proposition will be proved.

To prove (4.17), we check first the case $q=2$, that is,

$$
\lim _{n \rightarrow \infty} E\left(\left\|v_{G_{n}, Z}-v_{G, Z}\right\|_{L^{2}\left(K_{a}^{D}(t)\right)}^{2}\right)=0 .
$$

Indeed,

$$
\begin{aligned}
E\left(\left\|v_{G_{n}, Z}-v_{G, Z}\right\|_{L^{2}\left(K_{a}^{D}(t)\right)}^{2}\right) & =E\left(\left\|v_{G_{n}-G, Z}\right\|_{L^{2}\left(K_{a}^{D}(t)\right)}^{2}\right) \\
& \leq E\left(\left\|v_{G_{n}-G, Z}\right\|_{L^{2}\left(\mathbb{R}^{3}\right)}^{2}\right),
\end{aligned}
$$

and by the isometry property (3.1) of the stochastic integral, this is equal to

$$
\int_{0}^{t} d s \int_{\mathbb{R}^{3}} d \xi E\left(|\mathcal{F} Z(t-s)(\xi)|^{2}\right) \int_{\mathbb{R}^{3}} \mu(d \eta)\left|\mathcal{F}\left(G_{n}(t-s)-G(t-s)\right)(\xi-\eta)\right|^{2} .
$$

Notice that the integrand converges to 0 pointwise, and therefore the integral converges to 0 by the dominated convergence theorem, since the integrand is bounded by

$$
4 E\left(|\mathcal{F} Z(t-s)(\xi)|^{2}\right)|\mathcal{F} G(t-s)(\xi)|^{2}
$$


and by the Lipschitz property of $\sigma,(3.19)$ and (4.8),

$$
\begin{aligned}
& \int_{0}^{t} d s \int_{\mathbb{R}^{3}} d \xi E\left(|\mathcal{F} Z(t-s)(\xi)|^{2}\right) \int_{\mathbb{R}^{3}} \mu(d \eta)|\mathcal{F} G(t-s)(\xi-\eta)|^{2} \\
& \quad \leq \int_{0}^{t} d s E\left(\|Z(t-s)\|_{L^{2}\left(\mathbb{R}^{3}\right)}^{2}\right) \sup _{\xi \in \mathbb{R}^{3}} \int_{\mathbb{R}^{3}} \mu(d \eta)|\mathcal{F} G(t-s)(\xi-\eta)|^{2} \\
& \quad \leq C \int_{0}^{t} d s\left(1+E\left(\left\|u(t-s) 1_{K_{a}^{D}(t-s)}\right\|_{\left.L^{2}(\mathbb{R})\right)}^{2}\right)\right. \\
& \quad<+\infty
\end{aligned}
$$

This establishes (4.18).

In order to deduce (4.17) from (4.18), we use the fact that $L^{2}$-convergence (on $\left.\Omega \times K_{a}^{D}(t)\right)$ together with $L^{q}$-boundedness (for all $q$ ), implies $L^{q}$-convergence. In particular, it now suffices to show that

$$
\sup _{n>(a-1)^{-1}} E\left(\left\|v_{G_{n}, Z}^{t}\right\|_{L^{q}\left(K_{a}^{D}(t)\right)}^{q}\right)<+\infty .
$$

By (3.18) with $\mathcal{O}_{m}$ replaced by $K_{a}^{D}(t)$, we obtain using (4.15) that

$$
E\left(\left\|v_{G_{n}, Z}^{t}\right\|_{L^{q}\left(K_{a}^{D}(t)\right)}^{q}\right) \leq C \int_{0}^{t} d s E\left(\|Z(s)\|_{L^{q}\left(K_{a}^{D}(s)\right)}^{q}\right) .
$$

The right-hand side of (4.20) is finite by the Lipschitz property of $\sigma$ and (4.8). The proposition is proved.

The next lemma completes the study of the initial conditions needed in this section.

For a given real function $v$ defined on a bounded domain $\mathcal{O} \subset \mathbb{R}^{3}$ and for $\gamma \in] 0,1\left[\right.$, we denote by $\|v\|_{\mathcal{C} \gamma(\mathcal{O})}$ the $\gamma$-Hölder semi-norm on $\mathcal{O}$, that is

$$
\|v\|_{\mathcal{C}^{\gamma}(\mathcal{O})}=\sup _{x, y \in \mathcal{O} ; x \neq y} \frac{|v(x)-v(y)|}{|x-y|^{\gamma}} .
$$

Lemma 4.4 Let $v_{0}, \tilde{v}_{0}$ be real-valued functions. Assume that $v_{0} \in C^{2}\left(\mathbb{R}^{3}\right)$, $\Delta v_{0}$ is $\gamma_{1}$-Hölder continuous and $\tilde{v}_{0}$ is $\gamma_{2}$-Hölder continuous for some fixed $\left.\gamma_{1}, \gamma_{2} \in\right] 0,1[$. Then, for any $q \in[2, \infty[, t \in[0, T]$ and any bounded domain $\mathcal{O}$, there is a positive constant $C$ depending on $q, t$ and $\mathcal{O}$ such that for every $\left.\rho_{1} \in\right] 0, \gamma_{1}\left[\right.$ and $\left.\rho_{2} \in\right] 0, \gamma_{2}[$,

$$
\left\|\frac{d}{d t} G(t) * v_{0}\right\|_{\rho_{1}, q, \mathcal{O}} \leq C\left(\left\|v_{0}\right\|_{\rho_{1}, q, \mathcal{O}^{t}}^{q}+\left\|\Delta v_{0}\right\|_{\mathcal{C}^{\gamma_{1}}\left(\mathcal{O}^{t}\right)}^{q}\right)
$$


and

$$
\left\|G(t) * \tilde{v}_{0}\right\|_{\rho_{2}, q, \mathcal{O}} \leq C\left\|\tilde{v}_{0}\right\|_{\mathcal{C}^{\gamma_{2}\left(\mathcal{O}^{t}\right)}}^{q}
$$

Proof of Lemma 4.4: We start by checking the first inequality using the basic identity (4.11). Set $A(x)=\frac{1}{t}\left(v_{0} * G(t)\right)(x)$. Hölder's inequality with respect to the measure $G(t, d u)$ and the properties of $v_{0}$ yield

$$
\begin{aligned}
\|A(\cdot)\|_{\rho, q, \mathcal{O}}^{q} & \leq \frac{1}{t^{q}}\left(\int_{\mathbb{R}^{3}} G(t, d u)\right)^{q-1} \int_{\mathbb{R}^{3}} G(t, d u)\left\|v_{0}(\cdot-u)\right\|_{\rho, q, \mathcal{O}}^{q} \\
& \leq \frac{1}{t^{q}}\left(\int_{\mathbb{R}^{3}} G(t, d u)\right)^{q}\left\|v_{0}\right\|_{\rho_{1}, q, \mathcal{O}^{t}}^{q} \\
& =C\left\|v_{0}\right\|_{\rho_{1}, q, \mathcal{O}^{t}}^{q},
\end{aligned}
$$

for any $\rho \in] 0,1[$.

Let us now consider $B(x)=\int_{|z| \leq 1} \Delta v_{0}(x+t z) d z$. The Hölder-continuity property of $\Delta v_{0}$ implies that

$$
\begin{aligned}
\|B(\cdot)\|_{\rho, q, \mathcal{O}}^{q} & \leq\left\|\Delta v_{0}\right\|_{\mathcal{C}^{\gamma_{1}}\left(\mathcal{O}^{t}\right)}^{q} \int_{|z| \leq 1} d z \int_{\mathcal{O}} d x \int_{\mathcal{O}} d y|x-y|^{\left(\gamma_{1}-\rho-\frac{3}{q}\right) q} \\
& \leq C\left\|\Delta v_{0}\right\|_{\mathcal{C}^{\gamma_{1}\left(\mathcal{O}^{t}\right)}}^{q},
\end{aligned}
$$

if $\rho \in] 0, \gamma_{1}[$. This ends the proof of the first inequality in the lemma.

We now prove the second inequality. Set $R(x)=\left(G(t) * \tilde{v}_{0}\right)(x)$. As for $A(x)$, Hölder's inequality along with the Hölder-continuity of $\tilde{v}_{0}$ yield

$$
\begin{aligned}
\|R(\cdot)\|_{\rho, q, \mathcal{O}}^{q} & \leq\left\|\tilde{v}_{0}\right\|_{\mathcal{C}^{\gamma_{2}\left(\mathcal{O}^{t}\right)}}^{q} \int_{\mathcal{O}} d x \int_{\mathcal{O}} d y|x-y|^{\left(\gamma_{2}-\rho-\frac{3}{q}\right) q} \\
& \leq C\left\|\tilde{v}_{0}\right\|_{\mathcal{C}^{\gamma_{2}\left(\mathcal{O}^{t}\right)}}^{q},
\end{aligned}
$$

if $\rho \in] 0, \gamma_{2}[$. The proof of the lemma is now complete.

Remark 4.5 Assume that the hypotheses of Lemma 4.4 are satisfied. Then those of Lemma 4.2 hold for any $q \in[2, \infty[$. Consequently, the solution of the deterministic wave equation in dimension $d=3$,

$$
\left(\frac{\partial^{2}}{\partial t^{2}}-\Delta\right) w(t, x)=0,
$$

with initial conditions $v_{0}, \tilde{v}_{0}$, is a function $t \in[0, T] \mapsto w(t)$, such that each $w(t)$ takes values in $W^{\gamma, q}(\mathcal{O})$, for any $q \in\left[2, \infty[, \gamma \in] 0, \gamma_{1} \wedge \gamma_{2}[\right.$ and every bounded domain $\mathcal{O}$. In other words, $w(t)$ belongs to $W_{\text {loc }}^{\gamma, q}\left(\mathbb{R}^{3}\right)$. 
Indeed,

$$
w(t)=\frac{d}{d t} G(t) * v_{0}+G(t) * \tilde{v}_{0}
$$

and the above-mentioned two lemmas establish that

$$
\sup _{t \in[0, T]}\left\|\frac{d}{d t} G(t) * v_{0}+G(t) * \tilde{v}_{0}\right\|_{W^{\gamma, q}(\mathcal{O})}<\infty .
$$

We are now in a position to give the sample path behaviour in the $x$ variable of the solution of (4.2). In the next statement, $a \geq 1$ is a real number.

Theorem 4.6 Fix $q \in\left[2, \infty\left[\right.\right.$ and assume that $\tau(\beta, \delta):=\frac{2-\beta}{2} \wedge \frac{1+\delta}{2}>\frac{3}{q}$. Suppose also that

(a) $\sigma$ and $b$ are Lipschitz continuous functions,

(b) $v_{0}, \tilde{v}_{0}$ are real-valued functions, $v_{0} \in C^{2}\left(\mathbb{R}^{3}\right)$, and $\Delta v_{0}$ and $\tilde{v}_{0}$ are Hölder-continuous functions of order $\left.\gamma_{1}, \gamma_{2} \in\right] 0,1[$, respectively.

Set

$$
u^{0}(t, x)=\left(\frac{d}{d t} G(t) * v_{0}+G(t) * \tilde{v}_{0}\right)(x) .
$$

Then, for any $t \in[0, T]$,

$$
\sup _{n \geq 1} E\left(\left\|u_{n}(t)\right\|_{W^{\gamma, q}\left(K_{a}^{D}(t)\right)}^{q}\right) \leq C\left(1+\left\|u^{0}(t)\right\|_{W^{\gamma, q}\left(K_{a}^{D}(t)\right)}^{q}\right),
$$

for any $\gamma \in] 0, \gamma_{1} \wedge \gamma_{2} \wedge\left(\tau(\beta, \delta)-\frac{3}{q}\right)[$, and

$$
E\left(\|u(t)\|_{W^{\gamma, q}\left(K_{a}^{D}(t)\right)}^{q}\right) \leq C\left(1+\left\|u^{0}(t)\right\|_{W^{\gamma, q}\left(K_{a}^{D}(t)\right)}^{q}\right) .
$$

As a consequence,

$$
\sup _{t \in[0, T]} E\left(\|u(t)\|_{W^{\gamma, q}\left(K^{D}(t)\right)}^{q}\right) \leq C(1+\tilde{J}),
$$

where $K^{D}(t)=K_{1}^{D}(t)$ and

$$
\begin{gathered}
\tilde{J}=\left\|v_{0}\right\|_{W^{\gamma, q}\left(D^{T}\right)}^{q}+\left\|\Delta v_{0}\right\|_{L^{q}\left(D^{T}\right)}^{q}+\left\|\tilde{v}_{0}\right\|_{L^{q}\left(D^{T}\right)}^{q} \\
+\left\|\Delta v_{0}\right\|_{\mathcal{C}^{\gamma_{1}}\left(D^{T}\right)}^{q}+\left\|\tilde{v}_{0}\right\|_{\mathcal{C}^{\gamma_{2}}\left(D^{T}\right)}^{q} .
\end{gathered}
$$

In particular, for every $\gamma \in] 0, \gamma_{1} \wedge \gamma_{2} \wedge \tau(\beta, \delta)[$, there exists $q \in] 1, \infty[$ large enough so that

$$
\sup _{t \in[0, T]} E\left(\|u(t)\|_{W^{\gamma, q}\left(K^{D}(t)\right)}^{q}\right) \leq C(1+\tilde{J}) .
$$


Proof: For $n \geq 1$, consider the Picard iteration scheme corresponding to equation (4.12). That is, $u_{n}^{0}(t, x)=u^{0}(t, x)$, defined in (4.21) and, for $m \geq 1$,

$$
\begin{aligned}
u_{n}^{m}(t, x) 1_{K_{a}^{D}(t)}(x)= & u_{n}^{0}(t, x) 1_{K_{a}^{D}(t)}(x) \\
+ & 1_{K_{a}^{D}(t)}(x) \int_{0}^{t} \int_{\mathbb{R}^{3}} G_{n}(t-s, x-y) \\
& \times \sigma\left(u_{n}^{m-1}(s, y)\right) 1_{K_{a}^{D}(s)}(y) M(d s, d y) \\
+ & 1_{K_{a}^{D}(t)}(x) \int_{0}^{t} d s\left(G(t-s) * b\left(u_{n}^{m-1}(s, \cdot)\right) 1_{K_{a}^{D}(s)}(\cdot)\right)(x) .
\end{aligned}
$$

Set

$$
R_{n}^{m, \gamma, D}(t)=E\left(\left\|u_{n}^{m}(t)\right\|_{W^{\gamma, q}\left(K_{a}^{D}(t)\right)}^{q}\right) .
$$

Clearly, for $m \geq 1, R_{n, D}^{m, \gamma}(t) \leq C\left(\tilde{I}_{a}^{\gamma, D}(t)+T_{n}^{m, \gamma, D, 1}(t)+T_{n}^{m, \gamma, D, 2}(t)\right)$, with

$$
\begin{aligned}
& T_{n}^{m, \gamma, D, 1}(t)=E\left(\left\|v_{G_{n}, \sigma\left(u_{n}^{m-1}\right) 1_{K_{a}^{D}}}^{t}\right\|_{W^{\gamma, q}\left(K_{a}^{D}(t)\right)}^{q}\right), \\
& T_{n}^{m, \gamma, D, 2}(t)=E\left(\left\|J_{G, b\left(u_{n}^{m-1}\right) 1_{K_{a}^{D}}^{t}}\right\|_{W^{\gamma, q}\left(K_{a}^{D}(t)\right)}^{q}\right)
\end{aligned}
$$

and $\tilde{I}_{a}^{\gamma, D}(t)=\left\|u^{0}(t)\right\|_{W^{\gamma, q}\left(K_{a}^{D}(t)\right)}^{q}$. It follows from Remark 4.5 that for $\gamma \in$ ] $0, \gamma_{1} \wedge \gamma_{2}[$ and $a \geq 1$

$$
\sup _{t \in[0, T]} \tilde{I}^{\gamma, D}(t)<\infty .
$$

Let $g$ be a real-valued Lipschitz function. We shall show that the process

$$
\left\{g\left(u_{n}^{m-1}(t, \cdot)\right) 1_{K_{a}^{D}(t)}(\cdot), t \in[0, T]\right\}
$$

satisfies the following properties:

(i) $\sup _{n, m \geq 1} \sup _{t \in[0, T]} E\left(\left\|g\left(u_{n}^{m-1}(t)\right)\right\|_{L^{q}\left(K_{a}^{D}(t)\right)}^{q}\right)<\infty$,

(ii) for any $0 \leq s<t \leq T$,

$$
\begin{gathered}
\left.E\left(\left\|g\left(u_{n}^{m-1}(s)\right) 1_{K_{a}^{D}(s)}\right\|_{\gamma, q,\left(K_{a}^{D}(t)\right)}^{q}\right)^{(t-s)\left(1+\frac{1}{n}\right)}\right) \\
\leq C E\left(\left\|u_{n}^{m-1}(s)\right\|_{\gamma, q, K_{a}^{D}(s)}^{q}\right),
\end{gathered}
$$

(iii) for any $0 \leq s<t \leq T,|y| \leq t-s$,

$$
\begin{gathered}
E\left(\left\|g\left(u_{n}^{m-1}(s, \cdot-y)\right) 1_{K_{a}^{D}(s)}(\cdot-y)\right\|_{\gamma, q, K_{a}^{D}(t)}^{q}\right) \\
\leq C E\left(\left\|u_{n}^{m-1}(s)\right\|_{\gamma, q, K_{a}^{D}(s)}^{q}\right) .
\end{gathered}
$$


Indeed, $g$ has linear growth and using the same proof as in Theorem 4.1 with $G$ replaced by $G_{n}$, we see, as in (4.8), that

$$
\sup _{n, m \geq 1} \sup _{t \in[0, T]} E\left(\left\|u_{n}^{m}(t)\right\|_{L^{q}\left(K_{a}^{D}(t)\right)}^{q}\right)<\infty
$$

which proves (i).

To prove (ii), we use (4.15) and the Lipschitz property of $g$ to see that

$$
\begin{aligned}
& E\left(\left\|g\left(u_{n}^{m-1}(s)\right) 1_{K_{a}^{D}(s)}\right\|_{\gamma, q,\left(K_{a}^{D}(t)\right)^{(t-s)\left(1+\frac{1}{n}\right)}}^{q}\right) \\
& \quad=E\left(\left\|g\left(u_{n}^{m-1}(s)\right)\right\|_{\gamma, q,\left(K_{a}^{D}(t)\right)^{(t-s)\left(1+\frac{1}{n}\right)}}^{q}\right) \\
& \quad \leq C E\left(\left\|u_{n}^{m-1}(s)\right\|_{\gamma, q,\left(K_{a}^{D}(t)\right)^{(t-s)\left(1+\frac{1}{n}\right)}}^{q}\right) \\
& \quad=C E\left(\left\|u_{n}^{m-1}(s) 1_{K_{a}^{D}(s)}\right\|_{\gamma, q,\left(K_{a}^{D}(t)\right)^{(t-s)\left(1+\frac{1}{n}\right)}}^{q}\right) \\
& \quad \leq C E\left(\left\|u_{n}^{m-1}(s) 1_{K_{a}^{D}(s)}\right\|_{\gamma, q, K_{a}^{D}(s)}^{q}\right) .
\end{aligned}
$$

To prove (iii), let $0 \leq s<t \leq T$ and $|y| \leq t-s$. A change of variables yields

$$
\left\|g\left(u_{n}^{m-1}(s, \cdot-y)\right) 1_{K_{a}^{D}(s)}(\cdot-y)\right\|_{\gamma, q, K_{a}^{D}(t)}^{q} \leq\left\|g\left(u_{n}^{m-1}(s)\right) 1_{K_{a}^{D}(s)}(\cdot)\right\|_{\gamma, q,\left(K_{a}^{D}(t)\right)^{t-s}}^{q} .
$$

Then (iii) follows from the set inclusion $\left(K_{a}^{D}(t)\right)^{t-s} \subset K_{a}^{D}(s)$ and the Lipschitz property of $g$, in a similar manner as (ii).

Lemma 3.2, and more specifically (3.6), applied to the stochastic process $Z(s)=\sigma\left(u_{n}^{m-1}(s)\right) 1_{K_{a}^{D}(s)}, s \in[0, T], \mathcal{O}=K_{a}^{D}(t)$ and $\rho:=\gamma$ yields

$$
T_{n}^{m, \gamma, D, 1}(t) \leq C \int_{0}^{t} d s E\left(\left\|\sigma\left(u_{n}^{m-1}(s)\right) 1_{K_{a}^{D}(s)}\right\|_{W^{\gamma, q}\left(\left(K_{a}^{D}(t)\right)^{(t-s)\left(1+\frac{1}{n}\right)}\right.}^{q}\right) .
$$

By the properties (i), (ii) proved above with $g \equiv \sigma$, we obtain

$$
T_{n, D}^{m, \gamma, 1}(t) \leq C_{1}+C_{2} \int_{0}^{t} d s R_{n}^{m-1, \gamma, D}(s) .
$$

Hölder's inequality and property (iii) with $g \equiv b$ imply

$$
\begin{aligned}
& E\left(\left\|J_{G, b\left(u_{n}^{m-1}\right) 1_{K_{a}^{D}}}\right\|_{\gamma, q, K_{a}^{D}(t)}^{q}\right) \\
& \quad \leq C \int_{0}^{t} d s \int_{\mathbb{R}^{3}} G(t-s, d y) E\left(\left\|b\left(u_{n}^{m-1}(s, \cdot-y)\right) 1_{K_{a}^{D}(s)}(\cdot-y)\right\|_{\gamma, q, K_{a}^{D}(t)}^{q}\right) \\
& \quad \leq C \int_{0}^{t} d s E\left(\left\|u_{n}^{m-1}(s)\right\|_{\gamma, q, K_{a}^{D}(s)}^{q}\right) .
\end{aligned}
$$


Thus, owing to (i), we have

$$
T_{n}^{m, \gamma, D, 2}(t) \leq C_{1}+C_{2} \int_{0}^{t} d s R_{n}^{m-1, \gamma, D}(s) .
$$

Hence, the sequence of functions $\left.\left(R_{n}^{m, \gamma, D}(t), t \in[0, T], m \geq 1\right), \gamma \in\right] 0, \gamma_{1} \wedge$ $\gamma_{2} \wedge\left(\tau(\beta, \delta)-\frac{3}{q}\right)[$, satisfies the inequality

$$
R_{n}^{m, \gamma, D}(t) \leq C\left(1+\tilde{I}_{a}^{\gamma, D}(t)+\int_{0}^{t} d s R_{n}^{m-1, \gamma, D}(s)\right) .
$$

By Gronwall's lemma, we obtain

$$
\sup _{n, m \geq 1} R_{n}^{m, \gamma, D}(t) \leq C\left(1+\tilde{I}_{a}^{\gamma, D}(t)\right)
$$

and

$$
\sup _{n, m \geq 1} \sup _{t \in[0, T]} R_{n}^{m, \gamma, D}(t) \leq C\left(1+\sup _{t \in[0, T]} \tilde{I}_{a}^{\gamma, D}(t)\right)
$$

The Picard iterates satisfy

$$
\lim _{n \rightarrow \infty} \sup _{t \in[0, T]} E\left(\left\|\left(u_{n}^{m}(t)-u_{n}(t)\right) 1_{K^{D}(t)}\right\|_{L^{q}\left(\mathbb{R}^{3}\right)}^{q}\right)=0 .
$$

Indeed, this can be proved using the same arguments as those of the proof of Theorem 4.1.

Therefore, (4.22) follows from (4.29) and Fatou's lemma. Similarly, (4.23) follows from (4.22), Proposition 4.3 and Fatou's lemma.

To establish (4.24), we apply the same arguments as for the proof of (4.10) in Theorem 4.1 to check that

$$
\sup _{t \in[0, T]} E\left(\|u(t)\|_{\gamma, q, K^{D}(t)}^{q}\right) \leq C\left(\left\|v_{0}\right\|_{\gamma, q, D^{T}}+\left\|\Delta v_{0}\right\|_{\mathcal{C}^{\gamma_{1}\left(D^{T}\right)}}^{q}+\left\|\tilde{v}_{0}\right\|_{\mathcal{C}^{\gamma_{2}\left(D^{T}\right)}}^{q}\right) .
$$

This fact together with (4.10) finishes the proof.

Finally, (4.25) is a consequence of (4.24), since $q$ in (4.24) can be arbitrarily large.

An important consequence of Theorem 4.6 and the Sobolev embeddings is the following.

Corollary 4.7 Suppose that the hypotheses of Theorem 4.6 are satisfied. Then for any fixed $t>0$, a.s., the sample paths of $\left(u(t, x) 1_{K^{D}(t)}(x), x \in \mathbb{R}^{3}\right)$ are $\rho$-Hölder continuous with $\rho \in] 0, \gamma_{1} \wedge \gamma_{2} \wedge \tau(\beta, \delta)[$. 
We also notice that for any $q \in[2, \infty[$,

$$
\sup _{t \in[0, T]} \sup _{x, y \in K^{D}(t)} E\left(|u(t, x)-u(t, y)|^{q}\right) \leq C|x-y|^{\rho q},
$$

for any $\rho \in] 0, \gamma_{1} \wedge \gamma_{2} \wedge \tau(\beta, \delta)[$, and

$$
\sup _{t \in[0, T]} \sup _{x \in K^{D}(t)} E\left(|u(t, x)|^{q}\right)<\infty .
$$

Remark 4.8 Notice that Hölder regularity in the space variable is the same as that for the solution of the stochastic heat equation in any dimension $d \geq 1$ (see [26], Theorem 2.1).

\subsection{Regularity in time}

Our next aim is to analyze the behavior in time of the solution of (4.2). We begin by studying the properties of the term corresponding to the contribution of the initial conditions.

Throughout the section $D$ is an arbitrary bounded domain of $\mathbb{R}^{3}$.

Lemma 4.9 Let $v_{0}, \tilde{v}_{0}$ be real-valued functions.

(a) Let $v_{0} \in C^{2}\left(\mathbb{R}^{3}\right)$ be such that $\Delta v_{0}$ is $\gamma_{1}$-Hölder continuous for some $\left.\left.\gamma_{1} \in\right] 0,1\right]$. Then, the mapping $t \mapsto\left(\frac{d}{d t} G(t) * v_{0}\right)(x)(t \leq T)$ is also $\gamma_{1}$-Hölder continuous and

$$
\left.\sup _{x \in D} \| \frac{d}{d t} G(\cdot) * v_{0}\right)(x) \|_{\mathcal{C}^{\gamma_{1}([0, T])}} \leq C\left(\sup _{y \in D^{T}}\left|\nabla v_{0}(y)\right|+\left\|\Delta v_{0}\right\|_{\left.\mathcal{C}^{\gamma_{1}([0, T])}\right)} .\right.
$$

Therefore, its Hölder semi-norm is uniformly bounded in $x \in D$.

(b) Assume that $\tilde{v}_{0}$ is $\gamma_{2}$-Hölder continuous, for some $\left.\left.\gamma_{2} \in\right] 0,1\right]$. Then the mapping $t \mapsto\left(G(t) * \tilde{v}_{0}\right)(x)(t \leq T)$ is also $\gamma_{2}$-Hölder continuous and

$$
\sup _{x \in D}\left\|\left(G(\cdot) * \tilde{v}_{0}\right)(x)\right\|_{\mathcal{C}^{\gamma_{2}([0, T])}} \leq C\left(\sup _{x \in D^{T}}\left|\tilde{v}_{0}(x)\right|+\left\|\tilde{v}_{0}\right\|_{\mathcal{C}^{\gamma_{2}\left(D^{T}\right)}}\right) .
$$

Hence, its Hölder semi-norm is uniformly bounded in $x \in D$. 
Proof: We check (a) by studying each of the terms on the right-hand side of (4.11). Fix $t, \bar{t} \in[0, T]$ and $x \in D$. Then

$$
\begin{aligned}
I_{1}(t, \bar{t}, x) & :=\left|\frac{1}{t}\left(G(t) * v_{0}\right)(x)-\frac{1}{\bar{t}}\left(G(\bar{t}) * v_{0}\right)(x)\right| \\
& =\frac{1}{t}\left|\int_{\mathbb{R}^{3}} G(t, d u)\left(v_{0}(x-u)-v_{0}\left(x-\frac{\bar{t}}{t} u\right)\right)\right|,
\end{aligned}
$$

where we have used (2.2). The mean value theorem yields

$$
\begin{aligned}
I_{1}(t, \bar{t}, x) \mid & \leq \frac{1}{t} \int_{\mathbb{R}^{3}} G(t, d u) \sup _{y \in D^{T}}\left(\left|\nabla v_{0}(y)\right|\right)|u| \frac{|t-\bar{t}|}{t} \\
& \leq C \sup _{y \in D^{T}}\left|\nabla v_{0}(y)\right||t-\bar{t}|,
\end{aligned}
$$

since $G(t, \cdot)$ is concentrated on $B_{t}(0)$ and has total mass $t$. By the Höldercontinuity property of $\Delta v_{0}$,

$$
\begin{aligned}
I_{2}(t, \bar{t}, x) & :=\left|\int_{|y|<1}\left(\Delta v_{0}(x+t y)-\Delta v_{0}(x+\bar{t} y)\right) d y\right| \\
& \leq\left\|\Delta v_{0}\right\|_{\mathcal{C}^{\gamma_{1}\left(D^{T}\right)}}|t-\bar{t}|^{\gamma_{1}},
\end{aligned}
$$

The estimates (4.34), (4.35) and the identity (4.11) yields the result stated in (a).

Let us now prove (b). For any $t, \bar{t} \in[0, T]$,

$$
\begin{aligned}
I_{3}(t, \bar{t}, x) & :=\left|\left(G(t) * \tilde{v}_{0}\right)(x)-\left(G(\bar{t}) * \tilde{v}_{0}\right)(x)\right| \\
& =\left|\int_{\mathbb{R}^{3}} G(t, d u)\left(\tilde{v}_{0}(x-u)-\tilde{v}_{0}\left(x-\frac{\bar{t}}{t} u\right) \frac{\bar{t}}{t}\right)\right|,
\end{aligned}
$$

where, in the last equality, we have applied (2.2).

Hence

$$
I_{3}(t, \bar{t}, x) \leq I_{3}^{1}(t, \bar{t}, x)+I_{3}^{2}(t, \bar{t}, x),
$$

where

$$
\begin{aligned}
& I_{3}^{1}(t, \bar{t}, x)=\int_{\mathbb{R}^{3}} G(t, d u) \frac{\bar{t}}{t}\left|\tilde{v}_{0}(x-u)-\tilde{v}_{0}\left(x-u \frac{\bar{t}}{t}\right)\right|, \\
& I_{3}^{2}(t, \bar{t}, x)=\int_{\mathbb{R}^{3}} G(t, d u)\left|\tilde{v}_{0}(x-u)\right|\left|1-\frac{\bar{t}}{t}\right| .
\end{aligned}
$$


Using the Hölder-continuity property of $\tilde{v}_{0}$, we obtain

$$
\sup _{x \in D} I_{3}^{1}(t, \bar{t}, x) \leq C\left\|\tilde{v}_{0}\right\|_{\mathcal{C}^{\gamma_{2}\left(D^{T}\right)}}|t-\bar{t}|^{\gamma_{2}}
$$

Moreover, since $\tilde{v}_{0}$ is continuous and has compact support,

$$
\sup _{x \in D} I_{3}^{2}(t, \bar{t}, x) \leq C \sup _{x \in D^{T}}\left|\tilde{v}_{0}(x) \| t-\bar{t}\right|
$$

and statement (b) is established.

The next theorem provides upper bounds on $L^{q}$-moments of time increments of the solution of equation (4.2), uniformly over $x$ in bounded sets. We recall the notation $\tau(\beta, \delta)=\frac{2-\beta}{2} \wedge \frac{1+\delta}{2}$.

Theorem 4.10 Assume that

(a) $\sigma, b$ are Lipschitz functions,

(b) $v_{0}, \tilde{v}_{0}$ are real-valued functions, $v_{0} \in C^{2}\left(\mathbb{R}^{3}\right)$, and $\Delta v_{0}$ and $\tilde{v}_{0}$ are Höldercontinuous functions of order $\left.\left.\gamma_{1}, \gamma_{2} \in\right] 0,1\right]$, respectively.

Then, for any $t, \bar{t} \in[0, T]$ and each $q \in[2, \infty[$,

$$
\sup _{x \in D} E\left(|u(t, x)-u(\bar{t}, x)|^{q}\right) \leq C|t-\bar{t}|^{\alpha q}
$$

where $C$ is a positive constant and $\alpha \in] 0, \gamma_{1} \wedge \gamma_{2} \wedge \tau(\beta, \delta)[$.

Proof: Fix $x \in D$ and $q \in\left[2, \infty\left[\right.\right.$. Then $x \in K^{D}(t)$, for all $t \in[0, T]$, so by (4.2) with $a=1$,

$$
E\left(|u(t, x)-u(\bar{t}, x)|^{q}\right) \leq C \sum_{i=1}^{q} T_{i}(x, t, \bar{t}),
$$


where

$$
\begin{aligned}
& T_{1}(x, t, \bar{t})=\left|\left(\frac{d}{d t} G(t) * v_{0}\right)(x)-\left(\frac{d}{d t} G(\bar{t}) * v_{0}\right)(x)\right|^{q}, \\
& T_{2}(x, t, \bar{t})=\left|\left(G(t) * \tilde{v}_{0}\right)(x)-\left(G(\bar{t}) * \tilde{v}_{0}\right)(x)\right|^{q}, \\
& T_{3}(\cdot, t, \bar{t})=E\left(\mid \int_{0}^{t} \int_{\mathbb{R}^{3}} G(t-s, \cdot-y) \sigma(u(s, y)) 1_{K^{D}(s)}(y) M(d s, d y)\right. \\
& \left.\quad-\left.\int_{0}^{\bar{t}} \int_{\mathbb{R}^{3}} G(\bar{t}-s, \cdot-y) \sigma(u(s, y)) 1_{K^{D}(s)}(y) M(d s, d y)\right|^{q}\right) \\
& T_{4}(x, t, \bar{t})=E\left(\mid \int_{0}^{t} d s \int_{\mathbb{R}^{3}} G(t-s, d y) b(u(s, x-y)) 1_{K^{D}(s)}(x-y)\right. \\
& \left.\quad-\left.\int_{0}^{\bar{t}} d s \int_{\mathbb{R}^{3}} G(\bar{t}-s, d y) b(u(s, x-y)) 1_{K^{D}(s)}(x-y)\right|^{q}\right)
\end{aligned}
$$

Lemma 4.9 yields

$$
\sup _{x \in K^{D}(T)}\left(T_{1}(x, t, \bar{t})+T_{2}(x, t, \bar{t})\right) \leq C|t-\bar{t}|^{q \alpha_{1}},
$$

with $\left.\alpha_{1} \in\right] 0, \gamma_{1} \wedge \gamma_{2}[$.

Let $g$ be a real-valued Lipschitz function and set $\mathcal{I}:=] 0, \gamma_{1} \wedge \gamma_{2} \wedge \tau(\beta, \delta)[$. We next show that

(1) $\sup _{t \in[0, T]} E\left(\|g(u(t))\|_{L^{q}\left(K^{D}(t)\right)}^{q}\right)<\infty$;

(2) for any $\gamma \in \mathcal{I}, t \in] 0, T]$,

$$
\sup _{s \in[0, t]} E\left(\left\|g(u(s)) 1_{K^{D}(s)}\right\|_{\gamma, q,\left(K^{D}(t)\right)^{t-s}}^{q}\right)<\infty .
$$

Indeed, (1) follows from the linear growth property of $g$ and Theorem 4.1. To prove (2), we notice that since $\left(K^{D}(t)\right)^{t-s} \subset K^{D}(s)$, the arguments in the proof of property (ii) in Theorem 4.6 give

$$
\begin{gathered}
\sup _{s \in[0, t]} E\left(\left\|g(u(s)) 1_{K^{D}(s)}\right\|_{\gamma, q,\left(K^{D}(t)\right)^{t-s}}^{q}\right) \\
\leq C \sup _{s \in[0, t]} E\left(\|u(s)\|_{\gamma, q, K^{D}(s)}^{q}\right),
\end{gathered}
$$


and the conclusion follows from (4.25).

By properties (1) and (2) above, we see that the stochastic process $Z(s, y):=$ $g(u(s, y)) 1_{K^{D}(s)}(y),(s, y) \in[0, T] \times \mathbb{R}^{3}$, satisfies Assumption 3.4 with $\mathcal{O}:=D$ and arbitrarily large $q$. By applying Theorem 3.5, we conclude that for any $q \in[1, \infty[$,

$$
\sup _{x \in K^{D}(T)} T_{3}(x, t, \bar{t}) \leq C|t-\bar{t}|^{q \alpha_{2}}
$$

with $\alpha_{2} \in \mathcal{I}$.

Finally, we study the contribution of $T_{4}(x, t, \bar{t})$. Assume $t \leq \bar{t}$ and consider the decomposition

$$
T_{4}(x, t, \bar{t})=C\left(T_{4,1}(x, t, \bar{t})+T_{4,2}(x, t, \bar{t})\right),
$$

with

$$
\begin{aligned}
T_{4,1}(x, t, \bar{t})=E & \left(\left|\int_{t}^{\bar{t}} d s \int_{\mathbb{R}^{3}} G(\bar{t}-s, d y) b(u(s, x-y)) 1_{K^{D}(s)}(x-y)\right|^{q}\right), \\
T_{4,2}(x, t, \bar{t})=E & \left(\mid \int_{0}^{t} d s \int_{\mathbb{R}^{3}}(G(t-s, d y)-G(\bar{t}-s, d y)) b(u(s, x-y))\right. \\
& \left.\times\left. 1_{K^{D}(s)}(x-y)\right|^{q}\right) .
\end{aligned}
$$

Hölder's inequality, the restriction on the growth of $b$ and (4.31) imply that

$$
\begin{aligned}
\sup _{x \in D} T_{4,1}(x, t, \bar{t}) \leq & C|t-\bar{t}|^{q-1} \\
& \times \int_{t}^{\bar{t}} d s \int_{\mathbb{R}^{3}} G(\bar{t}-s, d y)\left(1+\sup _{t \in[0, T]} \sup _{y \in K^{D}(t)} E\left(|u(t, y)|^{q}\right)\right) \\
\leq & C|t-\bar{t}|^{q} .
\end{aligned}
$$

Notice that for $x \in D$ and $y \in B_{t-s}(0), x-y \in K^{D}(s)$, so $1_{K^{D}(s)}(x-y)$ can be removed from the expression for $T_{4,2}(x, t, \bar{t})$ when $x \in D$. We split the integral in the definition of $T_{4,2}(x, t, \bar{t})$ into a difference of integrals and then we apply the transformations $y \mapsto \frac{y}{t-s}$ and $y \mapsto \frac{y}{\bar{t}-s}$, respectively. We obtain

$$
\begin{aligned}
T_{4,2}(x, t, \bar{t})= & E\left(\mid \int_{0}^{t} d s \int_{B_{1}(0)} G(1, d y) b(u(s, x-(t-s) y))(t-s)\right. \\
& \left.-\left.\int_{0}^{t} d s \int_{B_{1}(0)} G(1, d y) b(u(s, x-(\bar{t}-s) y))(\bar{t}-s)\right|^{q}\right) .
\end{aligned}
$$


Hence $T_{4,2}(x, t, \bar{t}) \leq C\left(T_{4,2,1}(x, t, \bar{t})+T_{4,2,2}(x, t, \bar{t})\right)$, where

$$
\begin{aligned}
T_{4,2,1}(x, t, \bar{t})= & |t-\bar{t}|^{q} E\left(\left|\int_{0}^{t} d s \int_{B_{1}(0)} G(1, d y) b(u(s, x-(\bar{t}-s) y))\right|^{q}\right), \\
T_{4,2,2}(x, t, \bar{t})= & E\left(\mid \int_{0}^{t} d s(t-s) \int_{B_{1}(0)} G(1, d y)(b(u(s, x-(t-s) y))\right. \\
& \left.-b(u(s, x-(\bar{t}-s) y)))\left.\right|^{q}\right) .
\end{aligned}
$$

Clearly, from (4.31) and the linear growth property of $b$, it follows that

$$
\sup _{x \in K^{D}(t)} T_{4,2,1}(x, t, \bar{t}) \leq C|t-\bar{t}|^{q} .
$$

Moreover, by (4.30) and the Lipschitz property of $b$,

$$
\begin{aligned}
\sup _{x \in K^{D}(t)} T_{4,2,2}(x, t, \bar{t}) & \leq C|t-\bar{t}|^{\rho q} \int_{0}^{t} d s \int_{B_{1}(0)} G(1, d y)|y|^{\rho q} \\
& \leq C|t-\bar{t}|^{\rho q}
\end{aligned}
$$

for any $q \in[1, \infty[, \rho \in \mathcal{I}$.

Putting together (4.40) and (4.41), we obtain

$$
\sup _{x \in K^{D}(t)} T_{4,2}(x, t, \bar{t}) \leq C|t-\bar{t}|^{\rho q},
$$

for any $q \in[1, \infty[, \rho \in \mathcal{I}$. Together with (4.39), this yields

$$
\sup _{x \in K^{D}(t)} T_{4}(x, t, \bar{t}) \leq C|t-\bar{t}|^{\rho q}
$$

for each $q \in[1, \infty[, \rho \in \mathcal{I}$.

With the estimates (4.37), (4.38) and (4.43), we obtain (4.36).

We summarize the results of this section (Corollary 4.7 and Theorem 4.10) as follows.

Theorem 4.11 Assume that:

(a) The covariance of $F$ is of the form given in Assumption 2.4;

(b) the initial values $v_{0}, \tilde{v}_{0}$ are such that $v_{0} \in C^{2}\left(\mathbb{R}^{3}\right)$, and $\Delta v_{0}$ and $\tilde{v}_{0}$ are Hölder continuous with orders $\left.\left.\gamma_{1}, \gamma_{2} \in\right] 0,1\right]$, respectively;

(c) the coefficients $\sigma$ and $b$ are Lipschitz. 
Then for any $q \in\left[2, \infty[, \alpha \in] 0, \gamma_{1} \wedge \gamma_{2} \wedge \tau(\beta, \delta)[\right.$, there is $C>0$ such that for $(t, x),(\bar{t}, y) \in[0, T] \times D$,

$$
E\left(|u(t, x)-u(\bar{t}, y)|^{q}\right) \leq C(|t-\bar{t}|+|x-y|)^{\alpha q} .
$$

In particular, a.s., the stochastic process $(u(t, x),(t, x) \in[0, T] \times D)$ solution of (4.2) has $\alpha$-Hölder continuous sample paths, jointly in $(t, x)$.

Remark 4.12 Assume that the initial values $v_{0}, \tilde{v}_{0}$ satisfy the conditions given in (b) above. With Remark 4.5 and Lemma 4.9 we conclude that the solution of the deterministic inhomogeneous wave equation (take $\sigma \equiv 0$ in (4.2)) in dimension $d=3$ is $\alpha$-Hölder continuous, jointly in $(t, x)$, for any $\alpha \in] 0, \gamma_{1} \wedge \gamma_{2}[$.

\section{Sharpness of the results}

We devote this section to showing that the results of Theorem 4.11 on Hölder continuity in space and time are optimal. To do this, we consider the special case of equation (4.2) with vanishing initial conditions $v_{0}, \tilde{v}_{0}$, coefficients $\sigma \equiv 1, b \equiv 0$, and covariance function of the noise given by $f(x)=k_{\beta}(x)$, with $\beta \in] 0,2[$. In this case, the solution of equation (4.2) defines a stationary Gaussian process.

Theorem 5.1 Under the above assumptions on $v_{0}, \tilde{v}_{0}, \sigma$ and $b$, the solution $u(t, x)$ to the stochastic partial differential equation (4.2) has the following properties:

(a) Fix $t \in] 0, T]$ and a compact set $K \subset \mathbb{R}^{3}$. There is a constant $c_{1}>0$ such that for all $x, y \in K$,

$$
E\left(|u(t, x)-u(t, y)|^{2}\right) \geq c_{1}|x-y|^{2-\beta} .
$$

Consequently, a.s. the mapping $x \mapsto u(t, x)$ is not $\gamma$-Hölder continuous for $\gamma>\frac{2-\beta}{2}$, though it is for $\gamma<\frac{2-\beta}{2}$.

(b) Fix $x \in \mathbb{R}^{3}$ and $t_{0}>0$. There is a constant $c_{2}>0$ such that for all $t, \bar{t} \in\left[t_{0}, T\right]$ with $|\bar{t}-t|$ sufficiently small,

$$
E\left(|u(t, x)-u(\bar{t}-x)|^{2}\right) \geq c_{2}|t-\bar{t}|^{2-\beta}
$$

Hence, a.s., the mapping $t \mapsto u(t, x)$ is not $\gamma$-Hölder continuous for $\gamma>\frac{2-\beta}{2}$, while it is for $\gamma<\frac{2-\beta}{2}$. 
Proof. For any $x \in \mathbb{R}^{3}$, set $R(x)=E(u(t, x) u(t, 0))$. Since

$$
E\left(|u(t, x)-u(t, y)|^{2}\right)=2(R(0)-R(x-y)),
$$

it suffices to show that for any $x \in \mathbb{R}^{3}$,

$$
R(0)-R(x) \geq C_{1}|x|^{2-\beta},
$$

for some positive constant $C_{1}$. Without loss of generality, we may assume that $t=1$.

We remark that $R(0)-R(x)$ is a real number. Taking (2.5) into account and integrating with respect to $s$ yields

$$
\begin{aligned}
R(0)-R(x) & =\int_{0}^{1} d s \int_{\mathbb{R}^{3}} \mu(d \xi)\left(1-e^{i \xi \cdot x}\right)|\mathcal{F} G(1-s)(\xi)|^{2} \\
& =\frac{1}{2} \int_{\mathbb{R}^{3}} \frac{d \xi}{|\xi|^{5-\beta}}(1-\cos (\xi \cdot x))\left(1-\frac{\sin (2|\xi|)}{2|\xi|}\right),
\end{aligned}
$$

where $\xi \cdot x$ denotes the Euclidean inner product of the vectors $\xi$ and $x$.

If $|\xi|>1$, then $1-\frac{\sin (2|\xi|)}{2|\xi|} \geq \frac{1}{2}$. Thus, using the change of variables $w=|x| \xi$ and setting $e=\frac{x}{|x|}$ we obtain

$$
R(0)-R(x) \geq \frac{1}{4}|x|^{2-\beta} \int_{|w|>|x|} \frac{d w}{|w|^{5-\beta}}(1-\cos (w \cdot e)) .
$$

Because $x \in K$ and $K$ is compact, the last integral is bounded below by a positive constant, hence (5.1) is proved.

We now prove (5.2), assuming that $t_{0} \leq t<\bar{t} \leq T$. In this situation,

$$
E\left(|u(t, x)-u(\bar{t}, x)|^{2}\right)=T_{1}(t, \bar{t} ; x)+T_{2}(t, \bar{t} ; x),
$$

where

$$
\begin{aligned}
& T_{1}(t, \bar{t} ; x)=E\left(\int_{t}^{\bar{t}} \int_{\mathbb{R}^{3}} G(\bar{t}-s, x-y) M(d s, d y)\right)^{2}, \\
& T_{2}(t, \bar{t} ; x)=E\left(\int_{0}^{t}(G(\bar{t}-s, x-y)-G(t-s, x-y)) M(d s, d y)\right)^{2} .
\end{aligned}
$$

Using again the explicit formula (2.5) and integrating with respect to the variable $s$ yields

$$
\begin{aligned}
T_{1}(t, \bar{t} ; x) & =\int_{t}^{\bar{t}} d s \int_{\mathbb{R}^{3}} \frac{d \xi}{|\xi|^{3-\beta}}|\mathcal{F} G(\bar{t}-s)(\xi)|^{2} \\
& =\frac{1}{2} \int_{\mathbb{R}^{3}} \frac{d \xi}{|\xi|^{5-\beta}}\left((\bar{t}-t)-\frac{\sin (2(\bar{t}-t)|\xi|)}{2|\xi|}\right) .
\end{aligned}
$$


With the change of variables $w=(\bar{t}-t) \xi$, the last integral becomes

$$
|\bar{t}-t|^{3-\beta} \int_{\mathbb{R}^{3}} \frac{d w}{|w|^{5-\beta}}\left(1-\frac{\sin (2|w|)}{2|w|}\right),
$$

which clearly yields

$$
T_{1}(t, \bar{t} ; x) \geq C|\bar{t}-t|^{3-\beta} .
$$

A direct integration in the variable $s$ yields

$$
\begin{aligned}
T_{2}(t, \bar{t} ; x) & =\int_{0}^{t} d s \int_{\mathbb{R}^{3}} \frac{d \xi}{|\xi|^{3-\beta}}|(\mathcal{F} G(\bar{t}-s, \cdot)-\mathcal{F} G(t-s, \cdot))(\xi)|^{2} \\
& \geq \int_{0}^{t} d s \int_{|\xi| \geq(\bar{t}-t)^{-1}} \frac{d \xi}{|\xi|^{5-\beta}}(\sin ((\bar{t}-s)|\xi|)-\sin ((t-s)|\xi|))^{2} \\
& =\int_{|\xi| \geq(\bar{t}-t)^{-1}} \frac{d \xi}{|\xi|^{5-\beta}}(A(t, \bar{t}, \xi)+B(t, \bar{t}, \xi))
\end{aligned}
$$

where

$$
\begin{aligned}
& A(t, \bar{t}, \xi)=t(1-\cos ((\bar{t}-t)|\xi|))+\frac{\sin ((t+\bar{t})|\xi|)}{2|\xi|}(1-\cos ((\bar{t}-t)|\xi|)) \\
& B(t, \bar{t}, \xi)=\frac{\sin (2(\bar{t}-t)|\xi|)}{4|\xi|}-\frac{\sin ((t-\bar{t})|\xi|)}{2|\xi|} .
\end{aligned}
$$

Changing the variable $\xi$ into $w=(\bar{t}-t) \xi$ and bounding below the second term in $A(t, \bar{t}, \xi)$ yields

$$
\begin{aligned}
\int_{|\xi| \geq(\bar{t}-t)^{-1}} \frac{d \xi}{|\xi|^{5-\beta}} A(t, \bar{t}, \xi) & \geq|\bar{t}-t|^{2-\beta} \int_{|w|>1} \frac{d w}{|w|^{5-\beta}}(t(1-\cos |w|)-(\bar{t}-t)) \\
& \geq k_{1}|\bar{t}-t|^{2-\beta}-k_{2}|\bar{t}-t|^{3-\beta}
\end{aligned}
$$

(the last inequality uses that fact that $t \geq t_{0}>0$ ).

Similarly,

$$
\begin{aligned}
\int_{|\xi| \geq(\bar{t}-t)^{-1}} \frac{d \xi}{|\xi|^{5-\beta}} B(t, \bar{t}, \xi) & \geq \int_{|\xi| \geq(\bar{t}-t)^{-1}} \frac{d \xi}{|\xi|^{5-\beta}}\left(-\frac{1}{4|\xi|}-\frac{1}{2|\xi|}\right) \\
& \geq-\frac{3}{4}|\bar{t}-t|^{3-\beta} \int_{|w|>1} \frac{d w}{|w|^{5-\beta}} .
\end{aligned}
$$


Therefore,

$$
T_{2}(t, \bar{t} ; x) \geq k_{1}|\bar{t}-t|^{2-\beta}-k_{2}^{\prime}|\bar{t}-t|^{3-\beta} \geq \frac{k_{1}}{2}|\bar{t}-t|^{2-\beta},
$$

where the last lower bound holds whenever $|\bar{t}-t| \leq \frac{k_{1}}{2 k_{2}^{\prime}}$. This finishes the proof of (5.2).

The statements concerning absence of Hölder continuity follow from a well-known result on Gaussian stationary process (see for instance [2], Theorem 3.2). We only give some details for the space variable, since the arguments for the time variable are the same.

Assume by contradiction that for a fixed $t \in] 0, T]$, the sample paths $x \mapsto u(t, x)$ are $\gamma$-Hölder continuous for some $\gamma>\frac{2-\beta}{2}$, so that for any compact set $K \subset \mathbb{R}^{3}$, there is $0<C(\omega)<\infty$ with

$$
\sup _{x, y \in K, x \neq y} \frac{u(t, x)-u(t, y)}{|x-y|^{\gamma}}<C(\omega) .
$$

This implies that the Gaussian stochastic process

$$
\left(\frac{u(t, x)-u(t, y)}{|x-y|^{\gamma}}, x, y \in K, x \neq y\right)
$$

is finite a.s., and even, by Theorem 3.2 of [2], that

$$
E\left(\sup _{x, y \in K, x \neq y}\left(\frac{u(t, x)-u(t, y)}{|x-y|^{\gamma}}\right)^{2}\right)<\infty .
$$

In particular, there would exist a finite $K>0$ such that

$$
E\left(|u(t, x)-u(t, y)|^{2}\right) \leq K|x-y|^{2 \gamma} .
$$

This clearly contradicts (5.1).

Remark 5.2 Under the same assumptions as in Theorem 5.1, for any fixed $t \in] 0, T]$, a.s. the upper bounds on moments of increments of the process $\left(u(t, x), x \in \mathbb{R}^{3}\right)$ remains valid even for $\alpha=\frac{2-\beta}{2}$ (this, however, does not yield an improvement in the Hölder continuity of sample paths of this process).

Indeed, by (5.3), we can consider the decomposition

$$
R(0)-R(x)=r_{1}(x)+r_{2}(x)+r_{3}(x),
$$


with

$$
r_{1}(x)=\frac{1}{2} \int_{|\xi| \leq 1} \frac{d \xi}{|\xi|^{5-\beta}}(1-\cos (\xi \cdot x))\left(1-\frac{\sin (2|\xi|)}{2|\xi|}\right),
$$

and $r_{2}(x)$ (resp. $r_{3}(x)$ ) defined by the same expression, but with the domain of integration replaced by $|\xi||x| \geq 1$ (resp. $\left.1 \leq|\xi| \leq|x|^{-1}\right)$. Since $1-\cos (\xi$. $x) \leq \frac{1}{2}|\xi|^{2}|x|^{2}$, we clearly have $r_{1}(x) \leq C|x|^{2}$. Moreover, bounding above the products of trigonometric functions, we obtain

$$
r_{2}(x) \leq C \int_{|\xi||x| \geq 1} \frac{d \xi}{|\xi|^{5-\beta}}=C|x|^{2-\beta} .
$$

Finally, for $|x|$ small,

$$
\begin{aligned}
r_{3}(x) & \leq C|x|^{2} \int_{1 \leq|\xi| \leq|x|^{-1}} \frac{d \xi}{|\xi|^{3-\beta}}=C|x|^{2}\left(|x|^{-\beta}-1\right) \\
& \leq C|x|^{2-\beta} .
\end{aligned}
$$

Consequently,

$$
R(0)-R(x) \leq C|x|^{2-\beta}
$$

\section{Integrated increments of the covariance func- tion}

In this section, we prove technical results on integrals involving regularisations of the fundamental solution $G$ and one and two-dimensional increments of $f$. Each of these results plays a crucial role in the proofs of section 3 .

Lemma 6.1 For any $\alpha \in] 0,(2-\beta) \wedge 1[$, there is $C<\infty$ such that for all $x, y \in \mathbb{R}^{3}$,

$$
\sup _{n \geq 1} \sup _{s \in[0, T]} I_{n}(s, x, y) \leq C|x-y|^{\alpha}
$$

where

$$
I_{n}(s, x, y)=\int_{\mathbb{R}^{3}} d u \int_{\mathbb{R}^{3}} d v G_{n}(s, u) G_{n}(s, v)|D f(v-u, x-y)| .
$$

Proof. The structure of the function $f$ suggests the decomposition

$$
I_{n}(s, x, y) \leq I_{n}^{1}(s, x, y)+I_{n}^{2}(s, x, y)
$$


where $I_{n}^{1}(s, x, y)\left(\operatorname{resp} . I_{n}^{2}(s, x, y)\right)$ denotes the same expression but with the factor in absolute values replaced by

$\varphi(x-y+v-u)\left|D k_{\beta}(v-u, x-y)\right|, \quad\left(\operatorname{resp} . \quad|D \varphi(v-u, x-y)| k_{\beta}(v-u)\right)$.

Since the function $\varphi$ is bounded, we can apply Lemma 2.6(a) with $d=3$, $b:=\alpha, a:=3-(\alpha+\beta), u:=v-u, c:=|x-y|, x:=\frac{x-y}{|x-y|}$, to obtain

$$
\begin{aligned}
I_{n}^{1}(s, x, y) \leq & C|x-y|^{\alpha} \int_{\mathbb{R}^{3}} d u \int_{\mathbb{R}^{3}} d v G_{n}(s, u) G_{n}(s, v) \\
& \times \int_{\mathbb{R}^{3}} d w k_{\alpha+\beta}(|x-y| w+u-v)\left|D k_{3-\alpha}(w, \bar{e})\right|,
\end{aligned}
$$

where $\left.\bar{e}=\frac{x-y}{|x-y|}, \alpha+\beta \in\right] 0,2[$.

Observe that

$$
\begin{array}{rl}
\int_{\mathbb{R}^{3}} & d u \int_{\mathbb{R}^{3}} d v G_{n}(s, u) G_{n}(s, v) k_{\alpha+\beta}(|x-y| w+u-v) \\
\quad=\int_{\mathbb{R}^{3}} d \xi e^{i\langle\xi,|y-x| w\rangle}\left|\mathcal{F} G_{n}(s)(\xi)\right|^{2} k_{3-(\alpha+\beta)}(\xi) \\
\quad \leq \int_{\mathbb{R}^{3}} d \xi|\mathcal{F} G(s)(\xi)|^{2} k_{3-(\alpha+\beta)}(\xi)
\end{array}
$$

and this is uniformly bounded over $s \in[0, T]$ if $\alpha+\beta \in] 0,2[$ (see 2.4). On the other hand,

$$
\int_{\mathbb{R}^{3}} d w\left|D k_{3-\alpha}(w, e)\right|<\infty,
$$

if $\alpha \in] 0,1[$, as it is proved in Lemma 2.6(b). Consequently,

$$
\sup _{n \geq 1} \sup _{t \in[0, T]} I_{n}^{1}(t, x, y) \leq C|x-y|^{\alpha}
$$

with $\alpha \in] 0,(2-\beta) \wedge 1[$.

The mean value theorem, the properties of $\varphi$ and (2.4) imply that

$$
\begin{aligned}
I_{n}^{2}(t, x, y) & \leq C|x-y| \int_{\mathbb{R}^{3}} d u \int_{\mathbb{R}^{3}} d v G_{n}(s, u) G_{n}(s, v) k_{\beta}(v-u) \\
& \leq C|x-y| \int_{\mathbb{R}^{3}}|\mathcal{F} G(s)(\xi)|^{2} k_{3-\beta}(\xi) d \xi \\
& \leq C|x-y| .
\end{aligned}
$$

The estimates (6.2) and (6.3) establish (6.1). 
Lemma 6.2 For each $\alpha \in] 0,(2-\beta) \wedge(1+\delta)[$, there is $C<\infty$ such that for all $x, y \in \mathbb{R}^{3}$,

$$
\sup _{n \geq 1} \sup _{s \in[0, T]} J_{n}(s, x, y) \leq C|x-y|^{\alpha}
$$

where

$$
J_{n}(s, x, y)=\int_{\mathbb{R}^{3}} d u \int_{\mathbb{R}^{3}} d v G_{n}(s, u) G_{n}(s, v)\left|D^{2} f(v-u, y-x)\right| .
$$

Proof. Due to the structure of the covariance function $f$, it is easy to check that

$$
D^{2} f(v-u, y-x)=\sum_{1=1}^{3} \Delta^{2, i} f(v-u, y-x)
$$

where

$$
\begin{aligned}
\Delta^{2,1} f(v-u, y-x)= & \varphi(v-u) D^{2} k_{\beta}(v-u, y-x) \\
\Delta^{2,2} f(v-u, y-x)= & D \varphi(v-u, y-x)\left(D k_{\beta}(v-u, y-x)\right. \\
& \left.-D k_{\beta}(v-u, x-y)\right) \\
\Delta^{2,3} f(v-u, y-x)= & k_{\beta}(x-y+v-u) D^{2} \varphi(v-u, y-x) .
\end{aligned}
$$

Then, by the preceding decomposition,

$$
J_{n}(s, x, y) \leq \sum_{i=1}^{3} J_{n}^{i}(s, x, y),
$$

where $J_{n}^{i}(s, x, y)$ is defined in the same way as $J_{n}(s, x, y)$ but with $D^{2} f$ replaced by $\Delta^{2, i} f, i=1,2,3$. We now estimate the contribution of each of these terms.

Since $\varphi$ is bounded, applying Lemma 2.6(c) and (d) with $b:=\alpha, a:=$ $3-(\alpha+\beta), \alpha+\beta \in] 0,2[, u:=v-u, x:=y-x$, and (2.4), yields

$$
\begin{aligned}
J_{n}^{1}(s, x, y) \leq & |x-y|^{\alpha} \int_{\mathbb{R}^{3}} d u G_{n}(s, u) \int_{\mathbb{R}^{3}} d v G_{n}(s, v) \\
& \times \int_{\mathbb{R}^{3}} d w k_{\alpha+\beta}(|y-x| w+u-v)\left|D^{2} k_{3-\alpha}(w, e)\right| \\
\leq & |x-y|^{\alpha}\left(\sup _{x, y, w} \int_{\mathbb{R}^{3}} d u \int_{\mathbb{R}^{3}} d v G_{n}(s, u) G_{n}(s, v)\right. \\
& \left.\times k_{\alpha+\beta}(|y-x| w+u-v)\right) \int_{\mathbb{R}^{3}} d w\left|D^{2} k_{3-\alpha}(w, e)\right| \\
\leq & C|x-y|^{\alpha} .
\end{aligned}
$$


The properties of the function $\varphi$ in Assumption 2.4 imply that

$$
\begin{aligned}
J_{n}^{2}(s, x, y) \leq C|x-y| & \int_{\mathbb{R}^{3}} d u \int_{\mathbb{R}^{3}} d v G_{n}(s, u) G_{n}(s, v) \\
& \times\left|k_{\beta}(y-x+v-u)-k_{\beta}(x-y+v-u)\right| \\
\leq C|x-y| & \left(J_{n}^{2,-}(s, x, y)+J_{n}^{2,+}(s, x, y)\right),
\end{aligned}
$$

where

$$
J_{n}^{2, \pm}(s, x, y)=\int_{\mathbb{R}^{3}} d u \int_{\mathbb{R}^{3}} d v G_{n}(s, u) G_{n}(s, v)\left|D k_{\beta}(v-u, \pm(y-x))\right|,
$$

Notice that $J_{n}^{2,-}(s, x, y)$ coincides with the term $I_{n}^{1}(s, x, y)$ in Lemma 6.1 when $\varphi \equiv 1$; similarly, $J_{n}^{2,+}(s, x, y)=I_{n}^{1}(s, y, x)$ with $\varphi \equiv 1$. Consequently, (6.2) yields

$$
J_{n}^{2}(s, x, y) \leq C|x-y|^{1+\alpha},
$$

with $\alpha \in] 0,(2-\beta) \wedge 1[$.

The mean value theorem and the properties of $\varphi$ give

$$
\begin{aligned}
& \left|D^{2} \varphi(v-u, x-y)\right| \\
& \quad \leq|y-x| \int_{0}^{1} d \lambda|\nabla \varphi(v-u+\lambda(x-y))-\nabla \varphi(v-u-\lambda(x-y))| \\
& \quad \leq C|y-x|^{1+\delta} .
\end{aligned}
$$

Therefore, by (2.4),

$$
\begin{aligned}
J_{n}^{3}(s, x, y) & \leq C|y-x|^{1+\delta} \int_{\mathbb{R}^{3}} d u \int_{\mathbb{R}^{3}} d v G_{n}(s, u) G_{n}(s, v) k_{\beta}(x-y+v-u) \\
& \leq C|y-x|^{1+\delta} \int_{\mathbb{R}^{3}} d \xi k_{3-\beta}(\xi)|\mathcal{F} G(s)(\xi)|^{2} \\
& \leq C|y-x|^{1+\delta}
\end{aligned}
$$

Putting together the estimates (6.6)-(6.8), we obtain (6.4).

Lemma 6.3 For any $0 \leq s \leq t \leq \bar{t} \leq T$, set

$$
\nu_{1}^{n}(s, t, \bar{t})=\int_{\mathbb{R}^{3}} d u \int_{\mathbb{R}^{3}} d v G_{n}(t-s, u) G_{n}(t-s, v) f\left(\frac{\bar{t}-s}{t-s} v-u\right) \frac{\bar{t}-s}{t-s} .
$$

Then

$$
\sup _{n \geq 1} \sup _{0 \leq s \leq t \leq \bar{t} \leq T} \nu_{1}^{n}(s, t, \bar{t})<\infty .
$$


Proof. Consider the change of variables $v \mapsto \frac{\bar{t}-s}{t-s} v$. Then by Lemma 2.1,

$$
\begin{aligned}
\nu_{1}^{n}(s, t, \bar{t})= & \int_{\mathbb{R}^{3}} d u \int_{\mathbb{R}^{3}} d v G_{n}(t-s, u) G_{n}\left(t-s, \frac{t-s}{\bar{t}-s} v\right) \\
& \times f(v-u)\left(\frac{t-s}{\bar{t}-s}\right)^{2} \\
= & \int_{\mathbb{R}^{3}} d u \int_{\mathbb{R}^{3}} d v G_{n}(t-s, u) G_{n}(\bar{t}-s, v) f(v-u) .
\end{aligned}
$$

Using Assumption 2.4, the last integral is bounded by

$$
\|\varphi\|_{\infty} \int_{\mathbb{R}^{3}} d u \int_{\mathbb{R}^{3}} d v G_{n}(t-s, u) G_{n}(\bar{t}-s, v) k_{\beta}(v-u) .
$$

Consequently,

$$
\begin{aligned}
\nu_{1}^{n}(s, t, \bar{t}) & \leq C \int_{\mathbb{R}^{3}} d \xi \frac{\left|\mathcal{F} G_{n}(t-s)(\xi)\right|\left|\mathcal{F} G_{n}(\bar{t}-s)(\xi)\right|}{|\xi|^{3-\beta}} \\
& =C \int_{\mathbb{R}^{3}} d \xi \frac{|\mathcal{F} G(t-s)(\xi)|}{|\xi|^{(3-\beta) / 2}} \frac{\mid \mathcal{F} G(\bar{t}-s)(\xi))}{|\xi|^{(3-\beta) / 2}} .
\end{aligned}
$$

Apply the Cauchy-Schwarz inequality and (2.4) to see that this integral is bounded by a finite constant, uniformly in $s, t, \bar{t} \in[0, T]$.

The next two lemmas deal with time-scaled increments of the covariance density.

Lemma 6.4 For each $t, \bar{t} \in[0, T]$, with $t \leq \bar{t}$; set

$$
\begin{aligned}
\nu_{2}^{n}(t, \bar{t})= & \int_{0}^{t} d s \int_{\mathbb{R}^{3}} d u \int_{\mathbb{R}^{3}} d v G_{n}(t-s, u) G_{n}(t-s, v) \\
& \times\left|\left(\frac{\bar{t}-s}{t-s}\right)^{2} f\left(\frac{\bar{t}-s}{t-s}(v-u)\right)-\frac{\bar{t}-s}{t-s} f\left(\frac{\bar{t}-s}{t-s} v-u\right)\right| .
\end{aligned}
$$

Then

$$
\sup _{n \geq 1} \nu_{2}^{n}(t, \bar{t}) \leq C|\bar{t}-t|^{\alpha},
$$

for any $\alpha \in] 0,1[$ with $\alpha+\beta \in] 0,2[$. 
Proof. Consider the decomposition

$$
\nu_{2}^{n}(t, \bar{t}) \leq \nu_{2}^{n, 1}(t, \bar{t})+\nu_{2}^{n, 2}(t, \bar{t})
$$

where $\nu_{2}^{n, 1}(t, \bar{t})\left(\right.$ resp. $\left.\nu_{2}^{n, 2}(t, \bar{t})\right)$ is defined in the same way as $\nu_{2}^{n}(t, \bar{t})$, but with the expression in absolute values replaced by

$$
\left|\left(\frac{\bar{t}-s}{t-s}\right)^{2}-\frac{\bar{t}-s}{t-s}\right| f\left(\frac{\bar{t}-s}{t-s}(v-u)\right)
$$

and

$$
\frac{\bar{t}-s}{t-s}\left|f\left(\frac{\bar{t}-s}{t-s}(v-u)\right)-f\left(\frac{\bar{t}-s}{t-s} v-u\right)\right|,
$$

respectively.

The change of variables $(u, v) \mapsto \frac{\bar{t}-s}{t-s}(u, v)$ and Lemma 2.1 yield

$$
\begin{aligned}
\nu_{2}^{n, 1}(t, \bar{t}) & =|\bar{t}-t| \int_{0}^{t} \frac{d s}{\bar{t}-s} \int_{\mathbb{R}^{3}} d u \int_{\mathbb{R}^{3}} d v G_{n}(\bar{t}-s, u) G_{n}(\bar{t}-s, v) f(v-u) \\
& \leq C|\bar{t}-t| \int_{0}^{t} \frac{d s}{\bar{t}-s} \int_{\mathbb{R}^{3}} d \xi \frac{|\mathcal{F} G(\bar{t}-s)(\xi)|^{2}}{|\xi|^{3-\beta}} \\
& \leq C|\bar{t}-t|
\end{aligned}
$$

if $\beta \in] 0,2[$, where in the last inequality we have applied Lemma 2.3 with $b:=1$.

Consider the transformation $(u, v) \mapsto\left(u, \frac{\bar{t}-s}{t-s} v\right)$. Then

$$
\nu_{2}^{n, 2}(t, \bar{t})=\int_{0}^{t} d s \int_{\mathbb{R}^{3}} d u \int_{\mathbb{R}^{3}} d v G_{n}(t-s, u) G_{n}(\bar{t}-s, v) \mid D f\left(v-u,-\frac{\bar{t}-t}{t-s} u\right) .
$$

We are going to prove that

$$
\sup _{n \geq 1} \nu_{2}^{n, 2}(t, \bar{t}) \leq C|t-\bar{t}|^{\alpha},
$$

with $\alpha \in] 0,1[, \alpha+\beta \in] 0,2[$.

Notice that the structure of this term is similar to the integral analyzed in Lemma 6.1. Consider the decomposition

$$
\nu_{2}^{n, 2}(t, \bar{t}) \leq \nu_{2}^{n, 2,1}(t, \bar{t})+\nu_{2}^{n, 2,2}(t, \bar{t}),
$$


where $\nu_{2}^{n, 2,1}(t, \bar{t})\left(\right.$ resp. $\left.\nu_{2}^{n, 2,2}(t, \bar{t})\right)$ are as on the right-hand side of (6.11), but with the absolute value replaced by

$$
\left(\varphi\left(v-u-\frac{\bar{t}-t}{t-s} u\right)\left|D k_{\beta}\left(v-u-\frac{\bar{t}-t}{t-s} u\right)\right|,\right.
$$

and

$$
\left|D \varphi\left(v-u,-\frac{\bar{t}-t}{t-s} u\right)\right| k_{\beta}(v-u),
$$

respectively.

Since $\varphi$ is bounded, Lemma 2.6 (a) with $b=\alpha, a=3-(\alpha+\beta) c=\bar{t}-t$, $u:=v-u, x=-\frac{u}{t-s}$, yields

$$
\begin{aligned}
\nu_{2}^{n, 2,1}(t, \bar{t}) \leq C \mid t & -\left.\bar{t}\right|^{\alpha} \int_{0}^{t} d s \int_{\mathbb{R}^{3}} d u \int_{\mathbb{R}^{3}} d v G_{n}(t-s, u) G_{n}(\bar{t}-s, v) \\
& \times \int_{\mathbb{R}^{3}} d w k_{\alpha+\beta}((\bar{t}-t) w-(v-u))\left|D k_{3-\alpha}\left(w,-\frac{u}{t-s}\right)\right| .
\end{aligned}
$$

We next prove that if $\alpha+\beta \in] 0,2[$, then the integral above is bounded, uniformly in $n$ and $0 \leq t \leq \bar{t} \leq T$. For this, let $\nu_{2}^{n, 2,1,1}$ (resp. $\nu_{2}^{n, 2,1,2}$ ) be the same expression, but with $\mathbb{R}^{3}$ in the $d w$-integral replaced by $B_{3}(0)$ (resp. $\left.B_{3}(0)^{c}\right)$. For $\nu_{2}^{n, 2,1,1}$, break the absolute value in the $d w$-integral into the sum of two terms. Applying successively the changes of variables $w \mapsto w-\frac{u}{t-s}$ 
and $u \mapsto \frac{\bar{t}-s}{t-s} u$ and (2.3), the first term is bounded, by (2.4). Indeed

$$
\begin{aligned}
\int_{0}^{t} d s \int_{\mathbb{R}^{3}} d u \int_{\mathbb{R}^{3}} d v G_{n}(t-s, u) G_{n}(\bar{t}-s, v) \\
\quad \times \int_{|w| \leq 3} d w k_{\alpha+\beta}((\bar{t}-t) w-(v-u)) k_{3-\alpha}\left(\frac{u}{t-s}-w\right) \\
\leq \int_{0}^{t} d s \int_{\mathbb{R}^{3}} d u \int_{\mathbb{R}^{3}} d v G_{n}(t-s, u) G_{n}(\bar{t}-s, v) \\
\quad \times \int_{|w| \leq 5} d w k_{\alpha+\beta}\left((\bar{t}-t) w-v+\frac{\bar{t}-s}{t-s}\right) k_{3-\alpha}(w) \\
=\int_{0}^{t} d s \int_{\mathbb{R}^{3}} d u \int_{\mathbb{R}^{3}} d v G_{n}(\bar{t}-s, u) G_{n}(\bar{t}-s, v) \frac{t-s}{\bar{t}-s} \\
\quad \times \int_{|w| \leq 5} d w k_{\alpha+\beta}((\bar{t}-t) w-(v-u)) k_{3-\alpha}(w) \\
\leq \int_{|w| \leq 5} \frac{d w}{|w|^{3-\alpha}} \int_{0}^{t} d s \int_{\mathbb{R}^{3}} d \xi \frac{|\mathcal{F} G(\bar{t}-s)(\xi)|^{2}}{|\xi|^{3-(\alpha+\beta)}}
\end{aligned}
$$

which is bounded uniformly over $0 \leq t \leq \bar{t} \leq T$. if $\alpha+\beta \in] 0,2[$.

Similarly, the second term is

$$
\begin{aligned}
& \int_{0}^{t} d s \int_{\mathbb{R}^{3}} d u \int_{\mathbb{R}^{3}} d v G_{n}(t-s, u) G_{n}(\bar{t}-s, v) \\
& \quad \times \int_{|w| \leq 3} d w k_{\alpha+\beta}((\bar{t}-t) w-(v-u)) k_{3-\alpha}(w) \\
& \leq \int_{|w| \leq 3} \frac{d w}{|w|^{3-\alpha}} \int_{0}^{t} d s \int_{\mathbb{R}^{3}} d \xi \frac{|\mathcal{F} G(t-s)(\xi)||\mathcal{F} G(\bar{t}-s)(\xi)|}{|\xi|^{3-(\alpha+\beta)}}
\end{aligned}
$$

which is bounded uniformly over $0 \leq t \leq \bar{t} \leq T$, if $\alpha+\beta \in] 0,2[$.

Turning to $\nu_{2}^{n, 2,1,2}$, let

$$
\psi(\lambda)=k_{3-\alpha}\left(w-\lambda \frac{u}{t-s}\right), \quad \lambda \in[0,1] .
$$


Notice that since $|u| \leq(t-s)\left(1+\frac{1}{n}\right)$ and $|w|>3$, it follows that

$$
\left|w-\lambda \frac{u}{t-s}\right| \geq|| w\left|-\lambda\left(1+\frac{1}{n}\right)\right| \geq|w|-2>\frac{1}{3}|w| .
$$

Further, $\left|\psi^{\prime}(\lambda)\right| \leq C\left|w-\lambda \frac{u}{t-s}\right|^{\alpha-4}$. Thus,

$$
\begin{aligned}
\nu_{2}^{n, 2,1,2}(t, \bar{t}) \leq & \int_{0}^{t} d s \int_{\mathbb{R}^{3}} d u \int_{\mathbb{R}^{3}} d v G_{n}(t-s, u) G_{n}(\bar{t}-s, v) \\
& \left.\times \int_{|w|>3} d w \mid k_{\alpha+\beta}(\bar{t}-t) w+u-v\right) \mid \int_{0}^{1} d \lambda k_{4-\alpha}\left(w-\lambda \frac{u}{t-s}\right) \\
\leq & C \int_{|w|>3} \frac{d w}{|w|^{4-\alpha}} \int_{0}^{t} d s \int_{\mathbb{R}^{3}} d \xi \frac{|\mathcal{F} G(t-s)(\xi)||\mathcal{F} G(\bar{t}-s)(\xi)|}{|\xi|^{3-(\alpha+\beta)}}
\end{aligned}
$$

which is bounded uniformly over $0 \leq t \leq \bar{t} \leq T$, if $\alpha \in] 0,1[$ and $\alpha+\beta \in] 0,2[$.

Therefore, we have proved that

$$
\sup _{n \geq 1} \nu_{2}^{n, 2,1}(t, \bar{t}) \leq C|t-\bar{t}|^{\alpha}
$$

if $\alpha \in] 0,1[$ and $\alpha+\beta \in] 0,2[$.

By the properties of $\varphi$, we have

$$
\nu_{2}^{n, 2,2}(t, \bar{t}) \leq C|\bar{t}-t| \int_{0}^{t} d s \int_{\mathbb{R}^{3}} d \xi \frac{\left|\mathcal{F} G_{n}(t-s)(\xi)\right|\left|\mathcal{F} G_{n}(\bar{t}-s)(\xi)\right|}{|\xi|^{3-\beta}}
$$

and therefore

$$
\sup _{n \geq 1} \nu_{2}^{n, 2,2}(t, \bar{t}) \leq C|\bar{t}-t|
$$

The estimates (6.13) and (6.14) prove (6.12). Finally, (6.9) follows from (6.10) and (6.12).

The last lemma of this section gives information on the second-order increments of the time-scaled covariance function. Its proof is somewhat lengthy because we seek the best possible result: indeed, the case $\beta \in] 0,1[$ requires considerable effort, while the case $\beta \in[1,2[$ is not so complicated.

Lemma 6.5 For any $s, t, \bar{t} \in[0, T], s \leq t \leq \bar{t}, u, v \in \mathbb{R}^{3}$, we set

$$
\begin{array}{rl}
\Delta^{2} f(s, t, \bar{t}, u, v)=\left(\frac{\bar{t}-s}{t-s}\right)^{2} & f\left(\frac{\bar{t}-s}{t-s}(v-u)\right)-\frac{\bar{t}-s}{t-s} f\left(\frac{\bar{t}-s}{t-s} v-u\right) \\
& -\frac{\bar{t}-s}{t-s} f\left(v-\frac{\bar{t}-s}{t-s} u\right)+f(v-u)
\end{array}
$$


and

$$
\nu_{3}^{n}(t, \bar{t})=\int_{0}^{t} d s \int_{\mathbb{R}^{3}} d u \int_{\mathbb{R}^{3}} d v G_{n}(t-s, u) G_{n}(t-s, v)\left|\Delta^{2} f(s, t, \bar{t}, u, v)\right| .
$$

Then

$$
\sup _{n \geq 1} \nu_{3}^{n}(t, \bar{t}) \leq C|t-\bar{t}|^{\alpha},
$$

for any $\alpha \in] 0,(2-\beta) \wedge(1+\delta)[$.

Proof. Assume first that $\beta \in[1,2[$. In this case, we consider a decomposition of $\Delta^{2} f(s, t, \bar{t}, u, v)$ into first-order increments, which leads to the following decomposition:

$$
\nu_{3}^{n}(t, \bar{t})=\nu_{3}^{n, 1}(t, \bar{t})+\nu_{3}^{n, 2}(t, \bar{t})+\nu_{3}^{n, 3}(t, \bar{t}),
$$

where $\nu_{3}^{n, i}(t, \bar{t}), i=1,2,3$, is defined as $\nu_{3}^{n}(t, \bar{t})$, but with $\Delta^{2} f$ replaced by $\Delta^{2, i} f$, and

$$
\begin{aligned}
& \Delta^{2,1} f(s, t, \bar{t}, u, v)=\left|\left(\frac{\bar{t}-s}{t-s}\right)^{2} f\left(\frac{\bar{t}-s}{t-s}(v-u)\right)-\frac{\bar{t}-s}{t-s} f\left(\frac{\bar{t}-s}{t-s} v-u\right)\right|, \\
& \Delta^{2,2} f(s, t, \bar{t}, u, v)=\left|1-\frac{\bar{t}-s}{t-s}\right| f(v-u), \\
& \Delta^{2,3} f(s, t, \bar{t}, u, v)=\frac{\bar{t}-s}{t-s}\left|f(v-u)-f\left(v-\frac{\bar{t}-s}{t-s} u\right)\right| .
\end{aligned}
$$

Notice that $\nu_{3}^{n, 1}(t, \bar{t})$ is equal to $\nu_{2}^{n}(t, \bar{t})$ defined in Lemma 6.4. Thus, by (6.9),

$$
\sup _{n \geq 1} \nu_{3}^{n, 1}(t, \bar{t}) \leq C|t-\bar{t}|^{\alpha},
$$

for any $\alpha \in] 0,(2-\beta) \wedge 1[$.

For the term $\nu_{3}^{n, 2}(t, \bar{t})$, we have

$$
\nu_{3}^{n, 2}(t, \bar{t}) \leq C|t-\bar{t}| \int_{0}^{t} \frac{d s}{t-s} \int_{\mathbb{R}^{3}} d \xi \frac{|\mathcal{F} G(t-s)(\xi)|^{2}}{|\xi|^{3-\beta}} .
$$

Then, by Lemma 2.3 with $b=1$,

$$
\nu_{3}^{n, 2}(t, \bar{t}) \leq C|t-\bar{t}|
$$


for any $\beta \in] 0,2[$.

The analysis of $\nu_{3}^{n, 3}(t, \bar{t})$ does not differ very much from that of the term $\nu_{2}^{n, 2}(t, \bar{t})$ in the proof of Lemma 6.4. Indeed, the change of variables $(u, v) \mapsto$ $\left(\frac{\bar{t}-s}{t-s} u, v\right)$ and Lemma 2.1 yield

$$
\begin{aligned}
\nu_{3}^{n, 3}(t, \bar{t})= & \int_{0}^{t} d s \int_{\mathbb{R}^{3}} d u \int_{\mathbb{R}^{3}} d v G_{n}(\bar{t}-s, u) G_{n}(t-s, v) \\
& \times\left|D f\left(v-u, \frac{\bar{t}-t}{\bar{t}-s} u\right)\right| .
\end{aligned}
$$

Comparing this expression with (6.11), we observe that in the integrands, the roles of $t$ and $\bar{t}$ are exchanged. However, carrying on calculations similar to that for $\nu_{2}^{n, 2}(t, \bar{t})$ in the proof of Lemma 6.4, we encounter similar expressions, with $d s$ replaced by $\frac{d s}{t-s}$. Consequently, using Lemma 2.3 instead of (2.4), we obtain

$$
\sup _{n \geq 1} \nu_{3}^{n, 3} \leq C|t-\bar{t}|^{\alpha},
$$

for each $\alpha \in] 0,(2-\beta) \wedge 1[$. Notice that for $\beta \in[1,2],(2-\beta) \wedge 1=2-\beta$. Consequently, the estimate (6.16) follows in this case from (6.17)-(6.19).

Consider now the case $\beta \in] 0,1\left[\right.$. We decompose $\Delta^{2} f(s, t, \bar{t}, u, v)$ into the $\operatorname{sum} \sum_{i=1}^{3} \bar{\Delta}^{2, i} f(s, t, \bar{t}, u, v)$, with the definitions

$$
\begin{aligned}
\bar{\Delta}^{2,1} f(s, t, \bar{t}, u, v) & =\left(\left(\frac{\bar{t}-s}{t-s}\right)^{2}-2 \frac{\bar{t}-s}{t-s}+1\right) f(v-u) \\
& =\left(\frac{\bar{t}-t}{t-s}\right)^{2} f(v-u), \\
\bar{\Delta}^{2,2} f(s, t, \bar{t}, u, v) & =\left(\left(\frac{\bar{t}-s}{t-s}\right)^{2}-\frac{\bar{t}-s}{t-s}\right)\left(f\left(\frac{\bar{t}-s}{t-s}(v-u)\right)-f(v-u)\right), \\
\bar{\Delta}^{2,3} f(s, t, \bar{t}, u, v) & =\frac{\bar{t}-s}{t-s} \tilde{\Delta}^{2,3} f(s, t, \bar{t}, u, v),
\end{aligned}
$$


where

$$
\begin{aligned}
\tilde{\Delta}^{2,3} f(s, t, \bar{t}, u, v)= & \left(f\left(\frac{\bar{t}-s}{t-s}(v-u)\right)-f\left(\frac{\bar{t}-s}{t-s} v-u\right)-f\left(v-\frac{\bar{t}-s}{t-s} u\right)\right. \\
& +f(v-u)) .
\end{aligned}
$$

Then

$$
\nu_{3}^{n}(t, \bar{t}) \leq \sum_{i=1}^{3} \bar{\nu}_{3}^{n, i}(t, \bar{t}),
$$

with $\bar{\nu}_{3}^{n, i}(t, \bar{t})$ defined as $\nu_{3}^{n}(t, \bar{t})$, but with $\Delta^{2} f$ replaced by $\bar{\Delta}^{2, i} f, i=1,2,3$.

We start by estimating $\bar{\nu}_{3}^{n, 1}(t, \bar{t})$. We have

$$
\bar{\nu}_{3}^{n, 1}(t, \bar{t}) \leq C|t-\bar{t}|^{2} \int_{0}^{t} \frac{d s}{(t-s)^{2}} \int_{\mathbb{R}^{3}} d \xi \frac{|\mathcal{F} G(t-s)(\xi)|^{2}}{|\xi|^{3-\beta}} .
$$

Then, using Lemma 2.3 with $b=2$, we conclude that

$$
\sup _{n \geq 1} \bar{\nu}_{3}^{n, 1}(t, \bar{t}) \leq C|t-\bar{t}|^{2}
$$

if $\beta \in] 0,1[$.

We proceed now with $\bar{\nu}_{3}^{n, 2}(t, \bar{t})$, which we split into two terms:

$$
\begin{aligned}
\bar{\nu}_{3}^{n, 2,1} \leq & |t-\bar{t}| \int_{0}^{t} d s \int_{\mathbb{R}^{3}} d u \int_{\mathbb{R}^{3}} d v G_{n}(t-s, u) G_{n}(t-s, v) \frac{\bar{t}-s}{(t-s)^{2}} \\
& \times\left|f\left(\frac{\bar{t}-s}{t-s}(v-u)\right)-f\left(\frac{\bar{t}-s}{t-s} v-u\right)\right|, \\
\bar{\nu}_{3}^{n, 2,2} \leq & |t-\bar{t}| \int_{0}^{t} d s \int_{\mathbb{R}^{3}} d u \int_{\mathbb{R}^{3}} d v G_{n}(t-s, u) G_{n}(t-s, v) \frac{\bar{t}-s}{(t-s)^{2}} \\
& \times\left|f\left(\frac{\bar{t}-s}{t-s} v-u\right)-f(v-u)\right| .
\end{aligned}
$$

The change of variables $(u, v) \mapsto\left(u, \frac{\bar{t}-s}{t-s} v\right)$ and Lemma 2.1 yield

$$
\begin{aligned}
\bar{\nu}_{3}^{n, 2,1} \leq & |t-\bar{t}| \int_{0}^{t} \frac{d s}{t-s} \int_{\mathbb{R}^{3}} d u \int_{\mathbb{R}^{3}} d v G_{n}(t-s, u) G_{n}(\bar{t}-s, v) \\
& \times\left|D f\left(v-u,-\frac{\bar{t}-t}{t-s} u\right)\right| .
\end{aligned}
$$


Moreover,

$$
\begin{aligned}
\bar{\nu}_{3}^{n, 2,2} \leq & C|t-\bar{t}| \int_{0}^{t} \frac{d s}{(t-s)^{2}} \int_{\mathbb{R}^{3}} d u \int_{\mathbb{R}^{3}} d v G_{n}(t-s, u) G_{n}(t-s, v) \\
& \times\left|D f\left(v-u, \frac{\bar{t}-t}{t-s} v\right)\right| .
\end{aligned}
$$

Proceeding as for the analysis of the term $\nu_{3}^{n, 3}$, or $\nu_{2}^{n, 2}$ in Lemma 6.4 (observe the similarity of $\nu_{2}^{n, 2}$ in (6.11) with $\bar{\nu}_{3}^{n, 2,1}, \bar{\nu}_{3}^{n, 2,2}$ ), and using Lemma 2.3, we obtain

$$
\sup _{n \geq 1} \bar{\nu}_{3}^{n, 2}(t, \bar{t}) \leq C|t-\bar{t}|^{1+\rho_{1}}
$$

for any $\left.\rho_{1} \in\right] 0,1[$ (we recall that $\beta \in] 0,1[$ ).

The study of $\bar{\nu}_{3}^{n, 3}(t, \bar{t})$ is more intricate. Due to the product structure of the covariance function, we have the following decomposition

$$
\bar{\Delta}^{2,3} f(s, t, \bar{t}, u, v)=\frac{\bar{t}-s}{t-s} \sum_{i=1}^{4} \Delta^{2,3, i} f(s, t, \bar{t}, u, v),
$$

where

$$
\begin{aligned}
& \Delta^{2,3,1} f(s, t, \bar{t}, u, v)=\tilde{\Delta}^{2,3} k_{\beta}(s, t, \bar{t}, u, v) \varphi\left(\frac{\bar{t}-s}{t-s}(v-u)\right) \\
& \Delta^{2,3,2} f(s, t, \bar{t}, u, v)=D \varphi\left(\frac{\bar{t}-s}{t-s} v-u,-\frac{\bar{t}-t}{t-s} u\right) D k_{\beta}\left(v-u, \frac{\bar{t}-t}{t-s} v\right) \\
& \Delta^{2,3,3} f(s, t, \bar{t}, u, v)=D \varphi\left(v-\frac{\bar{t}-s}{t-s} u, \frac{\bar{t}-t}{t-s} v\right) D k_{\beta}\left(v-u,-\frac{\bar{t}-t}{t-s} u\right) \\
& \Delta^{2,3,4} f(s, t, \bar{t}, u, v)=\tilde{\Delta}^{2,3} \varphi(s, t, \bar{t}, u, v) k_{\beta}(v-u)
\end{aligned}
$$

Then $\bar{\nu}_{3}^{n, 3}(t, \bar{t}) \leq \sum_{i=1}^{4} \bar{\nu}_{3}^{n, 3, i}(t, \bar{t})$, where

$$
\begin{aligned}
\bar{\nu}_{3}^{n, 3, i}(t, \bar{t})= & \int_{0}^{t} d s \int_{\mathbb{R}^{3}} d u \int_{\mathbb{R}^{3}} d v G_{n}(t-s, u) G_{n}(t-s, v) \\
& \times \frac{\bar{t}-s}{t-s}\left|\Delta^{2,3, i} f(s, t, \bar{t}, u, v)\right| .
\end{aligned}
$$


We shall now analyze the contribution of each of these terms.

By Lemma $2.6(\mathrm{e})$, applied with $d=3, b:=\alpha, a:=3-(\alpha+\beta), c:=\bar{t}-t$, $u:=v-u, x:=-\frac{u}{t-s}, y:=\frac{v}{t-s}$,

$$
\bar{\nu}_{3}^{n, 3,1} \leq|\bar{t}-t|^{\alpha} I(t, \bar{t})
$$

where

$$
\begin{aligned}
I(t, \bar{t})= & \int_{0}^{t} d s \frac{\bar{t}-s}{t-s} \int_{\mathbb{R}^{3}} d u \int_{\mathbb{R}^{3}} d v G_{n}(t-s, u) G_{n}(t-s, v) \\
& \times \int_{\mathbb{R}^{3}} d w k_{\alpha+\beta}(v-u-(\bar{t}-t) w)\left|\bar{D}^{2} k_{3-\alpha}\left(w,-\frac{u}{t-s}, \frac{v}{t-s}\right)\right| .
\end{aligned}
$$

Our next objective is to check that $\sup _{0 \leq t \leq \bar{t} \leq T} I(t, \bar{t})<\infty$. This will be carried out by taking into account first the small values of $w(|w| \leq 5)$, and then the large ones.

Set $\bar{t}-t=h$ and split the last absolute value above into four terms, so that the above integral when $w$ is integrated on the set $|w| \leq 5$ equals to $\sum_{j=1}^{4} I_{j}(t, \bar{t})$.

Clearly,

$$
\begin{aligned}
I_{1}(t, \bar{t}):= & \int_{0}^{t} d s \frac{\bar{t}-s}{t-s} \int_{\mathbb{R}^{3}} d u \int_{\mathbb{R}^{3}} d v G_{n}(t-s, u) G_{n}(t-s, v) \\
& \times \int_{|w|<5} d w k_{\alpha+\beta}(v-u-(\bar{t}-t) w) k_{3-\alpha}(w) \\
\leq & \left(\int_{|w|<5} \frac{d w}{|w|^{3-\alpha}}\right) \int_{0}^{t} d s \frac{\bar{t}-s}{t-s} \int_{\mathbb{R}^{3}} d \xi \frac{\left.\mathcal{F} G(t-s)(\xi)\right|^{2}}{|\xi|^{3-(\alpha+\beta)}}<\infty,
\end{aligned}
$$

if $\alpha+\beta \in] 0,2[, \alpha>0$.

The analysis of $I_{j}(t, \bar{t}), j=2,3,4$, are all similar, so we only give the details for

$$
\begin{aligned}
I_{4}(t, \bar{t})= & \int_{0}^{t} d s \frac{\bar{t}-s}{t-s} \int_{\mathbb{R}^{3}} d u \int_{\mathbb{R}^{3}} d v G_{n}(t-s, u) G_{n}(t-s, v) \\
& \times \int_{|w|<5} d w k_{\alpha+\beta}(v-u-h w) k_{3-\alpha}\left(w+\frac{v-u}{t-s}\right) .
\end{aligned}
$$


Consider the change of variables $\bar{w}=w+\frac{v-u}{t-s}$. Then

$$
\begin{aligned}
I_{4}(t, \bar{t}) \leq & \int_{|w|<7} \frac{d w}{|w|^{3-\alpha}} \int_{0}^{t} d s \frac{\bar{t}-s}{t-s} \int_{\mathbb{R}^{3}} d u \int_{\mathbb{R}^{3}} d v G_{n}(t-s, u) G_{n}(t-s, v) \\
& \times k_{\alpha+\beta}\left(h w+\frac{\bar{t}-s}{t-s}(v-u)\right) .
\end{aligned}
$$

Apply the change of variables $(u, v) \mapsto \frac{\bar{t}-s}{t-s}(u, v)$. Then, by Lemma 2.1,

$$
\begin{aligned}
I_{4}(t, \bar{t}) \leq & \int_{|w|<7} \frac{d w}{|w|^{3-\alpha}} \int_{0}^{t} d s \frac{t-s}{\bar{t}-s} \int_{\mathbb{R}^{3}} d u \int_{\mathbb{R}^{3}} d v G(\bar{t}-s, u) G(\bar{t}-s, v) \\
& \times k_{\alpha+\beta}(h w+(v-u)) \\
\leq & C \int_{0}^{\bar{t}} d s \int_{\mathbb{R}^{3}} d \xi \frac{|\mathcal{F} G(t-s)(\xi)|^{2}}{|\xi|^{3-(\alpha+\beta)}}
\end{aligned}
$$

which is bounded, uniformly over $0 \leq t \leq \bar{t} \leq T$, if $\alpha+\beta \in] 0,2[, \alpha>0$.

Therefore, we have proved that the contribution to $I(t, \bar{t})$ of the term in which the $d w$-integral is restricted to $\{|w|<5\}$ is finite.

We end the proof by checking that $I_{5}(t, \bar{t})<\infty$, where $I_{5}(t, \bar{t})$ is defined in the same way as $I(t, \bar{t})$ but with the $d w$-integral restricted to the domain $\{|w| \geq 5\}$.

Define

$$
\Psi(\lambda, \mu)=k_{3-\alpha}\left(w-\lambda \frac{u}{t-s}+\mu \frac{v}{t-s}\right), \lambda, \mu \in[0,1],
$$

so that

$$
\begin{aligned}
\bar{D}^{2} k_{3-\alpha}\left(w,-\frac{u}{t-s}, \frac{v}{t-s}\right) & =\Psi(1,1)-\Psi(0,1)-\Psi(1,0)+\Psi(0,0) \\
& =\int_{0}^{1} d \lambda \int_{0}^{1} d \mu \frac{\partial^{2} \Psi}{\partial \lambda \partial \mu}(\lambda, \mu) .
\end{aligned}
$$

A simple computation shows that

$$
\left|\frac{\partial^{2} \Psi}{\partial \lambda \partial \mu}(\lambda, \mu)\right| \leq C k_{5-\alpha}\left(w-\lambda \frac{u}{t-s}+\mu \frac{v}{t-s}\right) .
$$


Consequently

$$
\begin{aligned}
I_{5}(t, \bar{t}):= & \int_{0}^{t} d s \frac{\bar{t}-s}{t-s} \int_{\mathbb{R}^{3}} d u \int_{\mathbb{R}^{3}} d v G_{n}(t-s, u) G_{n}(t-s, v) \\
& \times \int_{|w| \geq 5} d w k_{\alpha+\beta}(v-u-h w) \tilde{D}^{2} k_{3-\alpha}\left(w,-\frac{u}{t-s}, \frac{v}{t-s}\right) \\
\leq & C \int_{0}^{t} d s \frac{\bar{t}-s}{t-s} \int_{\mathbb{R}^{3}} d u \int_{\mathbb{R}^{3}} d v G_{n}(t-s, u) G_{n}(t-s, v) \\
& \times \int_{|w| \geq 5} d w k_{\alpha+\beta}(v-u-h w) \\
& \times \int_{0}^{1} d \lambda \int_{0}^{1} d \mu k_{5-\alpha}\left(w-\lambda \frac{u}{t-s}+\mu \frac{v}{t-s}\right) .
\end{aligned}
$$

We can give a lower bound of $\left|w-\lambda \frac{u}{t-s}+\mu \frac{v}{t-s}\right|$, independent of $u, v$ on the set $\{|w| \geq 5\}$. Indeed, by the triangle inequality

$$
\begin{aligned}
\left|w-\lambda \frac{u}{t-s}+\mu \frac{v}{t-s}\right| & \geq|| w|-| \lambda \frac{u}{t-s}-\mu \frac{v}{t-s}|| \\
& \geq|| w|-2(\lambda+\mu)| \\
& \geq|w|-4 \geq \frac{|w|}{5} .
\end{aligned}
$$

Hence,

$$
\begin{aligned}
I_{5}(t, \bar{t}) \leq & C \int_{|w| \geq 5} \frac{d w}{|w|^{5-\alpha}} \int_{0}^{t} d s \frac{\bar{t}-s}{t-s} \int_{\mathbb{R}^{3}} d u \int_{\mathbb{R}^{3}} d v G_{n}(t-s, u) G_{n}(t-s, v) \\
& \times k_{\alpha+\beta}(v-u-h w) \\
\leq & C \int_{|w| \geq 5} \frac{d w}{|w|^{5-\alpha}} \int_{0}^{t} d s \frac{\bar{t}-s}{t-s} \int_{\mathbb{R}^{3}} d \xi \frac{|\mathcal{F} G(t-s)(\xi)|^{2}}{|\xi|^{3-(\alpha+\beta)}} .
\end{aligned}
$$

The $d w$-integral is finite for any $\alpha \in] 0,2[$ and, by Lemma 2.3, the $d s d \xi$ integral is also finite for $\alpha+\beta \in] 0,2\left[\right.$. Consequently, $I_{5}(t, \bar{t})$ is finite whenever $\alpha+\beta \in] 0,2[$; therefore

$$
\sup _{n \geq 1} \bar{\nu}_{3}^{n, 3,1}(t, \bar{t}) \leq C|t-\bar{t}|^{\alpha}
$$


for any $\alpha \in] 0,2-\beta[$.

By the properties of $\varphi$, we have

$$
\begin{aligned}
\bar{\nu}_{3}^{n, 3,2}(t, \bar{t}) \leq & C \int_{0}^{t} d s \frac{\bar{t}-s}{t-s} \int_{\mathbb{R}^{3}} d u \int_{\mathbb{R}^{3}} d v G_{n}(t-s, u) G_{n}(t-s, v) \\
& \times \frac{|t-\bar{t}|}{t-s}|u|\left|D k_{\beta}\left(v-u, \frac{\bar{t}-t}{t-s} v\right)\right| \\
\leq & C|t-\bar{t}| \int_{0}^{t} d s \frac{1}{t-s} \int_{\mathbb{R}^{3}} d u \int_{\mathbb{R}^{3}} d v G_{n}(t-s, u) G_{n}(t-s, v) \\
& \times\left|D k_{\beta}\left(v-u, \frac{\bar{t}-t}{t-s} v\right)\right| .
\end{aligned}
$$

In the last inequality, we have used the fact that $|u| \leq(\bar{t}-s)\left(1+\frac{1}{n}\right)$.

We can study the contribution of this last integral with similar arguments as those used in the analysis of the term $\nu_{2}^{n, 2,1}(t, \bar{t})$ in the proof of Lemma 6.4 , concluding that

$$
\sup _{n \geq 1} \bar{\nu}_{3}^{n, 3,2} \leq C|t-\bar{t}|^{1+\rho_{3}}
$$

for any $\left.\rho_{3} \in\right] 0,(2-\beta) \wedge 1[($ see $(6.13))$. Clearly, the same bound holds for the term $\bar{\nu}_{3}^{n, 3,3}$.

By the assumptions on $\varphi$, we have

$$
\begin{gathered}
\left|\varphi\left(\frac{\bar{t}-s}{t-s}(v-u)\right)-\varphi\left(\frac{\bar{t}-s}{t-s} v-u\right)-\varphi\left(v-\frac{\bar{t}-s}{t-s} u\right)+\varphi(v-u)\right| \\
\leq \int_{0}^{1} d \lambda\left|\nabla \varphi\left(\frac{\bar{t}-s}{t-s} v-u+\lambda \frac{t-\bar{t}}{t-s} u\right)-\nabla \varphi\left(v-u+\lambda \frac{t-\bar{t}}{t-s} u\right)\right| \\
\times \frac{|t-\bar{t}|}{t-s}|u| \leq C \frac{|t-\bar{t}|^{1+\delta}}{(t-s)^{1+\delta}}|u||v|^{\delta} .
\end{gathered}
$$

It follows that

$$
\begin{aligned}
\bar{\nu}_{3}^{n, 3,4}(t, \bar{t}) \leq & C|t-\bar{t}|^{1+\delta} \int_{0}^{t} d s \frac{\bar{t}-s}{t-s} \int_{\mathbb{R}^{3}} d u \int_{\mathbb{R}^{3}} d v G_{n}(t-s, u) G_{n}(t-s, v) \\
& \times \frac{|u||v|^{\delta}}{(t-s)^{1+\delta}} k_{\beta}(v-u) .
\end{aligned}
$$


The fraction with $u$ and $v$ is bounded by $2^{1+\delta}$. Therefore

$$
\bar{\nu}_{3}^{n, 3,4}(t, \bar{t}) \leq C|t-\bar{t}|^{1+\delta} \int_{0}^{t} d s \frac{\bar{t}-s}{t-s} \int_{\mathbb{R}^{3}} d \xi \frac{|\mathcal{F} G(t-s)(\xi)| \mid \overline{\mathcal{F} G(t-s)(\xi)}}{|\xi|^{3-\beta}}
$$

and by Lemma 2.3 ,

$$
\sup _{n \geq 1} \bar{\nu}_{3}^{n, 3,4}(t, \bar{t}) \leq C|t-\bar{t}|^{1+\delta}
$$

if $\beta \in] 0,2[$.

Summarising the results obtained in (6.22)-(6.24) yields

$$
\sup _{n \geq 1} \bar{\nu}_{3}^{n, 3}(t, \bar{t}) \leq C|t-\bar{t}|^{\alpha}
$$

with $\alpha \in] 0,(2-\beta) \wedge(1+\delta)[$.

Finally, by (6.20), (6.21) and (6.25), we conclude that

$$
\sup _{n \geq 1} \nu_{3}^{n}(t, \bar{t}) \leq C|t-\bar{t}|^{\alpha},
$$

wiht $\alpha \in] 0,(2-\beta) \wedge(1+\delta)[$, when $\beta \in] 0,1[$. The proof of the lemma is complete.

Acknowledgment: The second named author is pleased to thank the Institut de Mathématiques of the Ecole Polytechnique Fédérale de Lausanne for its hospitality and financial support during a visit where part of this work was carried out.

\section{References}

[1] R.A. Adams, J.J.F. Fournier: Sobolev Spaces. 2nd Edition. Pure and Applied Mathematics Series. Elsevier, 2003.

[2] R.J. Adler: An Introduction to Continuity, Extrema, and Related Topics for General Gaussian Processes. Institute of Mathematical Statistics Lecture Notes-Monographs Series, Vol 12, 1990.

[3] E. Cabaña: The vibrating string forced by white noise. Z. Wahrscheinlichkeitstheorie und Verw. Gebiete 15, 111-130, 1970.

[4] R. Carmona, D. Nualart: Random nonlinear wave equations: smoothness of the solutions. Probab. Theory Related Fields 79, 469-508 (1988). 
[5] R.C. Dalang, N.E. Frangos: The stochastic wave equation in two spatial dimensions. Annals of Probab. 26, 1, 187-212, 1998.

[6] R.C. Dalang: Extending the martingale measure stochastic integral with applications to spatially homogeneous spde's. Electronic J. of Probability, Vol 4, 1999.

[7] R.C. Dalang, C. Mueller: Some non-linear SPDE's that are second order in time. Electronic J. of Probability, Vol 8, 1, 1-21, 2003.

[8] R.C. Dalang, M. Sanz-Solé: Regularity of the sample paths of a class of second-order spde's. J. Funct. Anal. 227, 2, 304-337 (2005).

[9] J. Dieudonné: Foundations of Modern Analysis. Pure and Applied Mathematics, Vol. X. Academic Press, New York-London, 1960.

[10] W.F. Donoghue: Distributions and Fourier transforms. Academic Press, New York, 1969.

[11] L. Hörmander: Lectures on Nonlinear Hyperbolic Differential Equations. Springer Verlag, 1997.

[12] A. Karkzewska, J. Zabczyk: "Stochastic PDE's with function-valued solutions", in Clément Ph., den Hollander F., van Neerven J. and de Pagter B. (Eds), "Infinite-dimensional stochastic analysis", Proceedings of the Colloquium of the Royal Netherlands Academy of Arts and Sciences, 1999, Amsterdam.

[13] N.V. Krylov: An analytic approach to spde's. In: Stochastic Partial Differential Equations: Six Perspectives (R.A. Carmona, B. Rozovskii, Eds.), pp. 185-242. Mathematical Surveys and Monographs, Vol 64, American Mathematical Society, 1999.

[14] D. Márquez, M. Mellouk, M. Sarrà: On stochastic partial differential equations with spatially correlated noise: smoothness of the law. Stoch. Proc. Appl. 93, 269-284 (2001).

[15] M. Métivier: Semimartingales, a Course on Stochastic Processes. de Gruyter Studies in Mathematics 2. Walter de Gruyter, 1982.

[16] A. Millet, M. Sanz-Solé: A stochastic wave equation in two space dimensions: smoothness of the law. Annals of Probab. 27, 803-844, 1999.

[17] A. Millet, M. Sanz-Solé: Approximation and support theorem for a wave equation in two space dimensions. Bernoulli 6, 5, 887-915, 2000. 
[18] L. Mytnik, E. Perkins, A. Sturm: On pathwise uniqueness for stochastic heat equations with non-Lipschitz coefficients. Prepublication (2005).

[19] S. Peszat, J. Zabczyk: Nonlinear stochastic wave and heat equations. Probab. Theory Related Fields, 116, 421-443, 2000.

[20] S. Peszat, The Cauchy problem for a nonlinear stochastic wave equation in any dimension, J. Evol. Equ., 2, no. 3, 383-394.(2002).

[21] Ll. Quer-Sardanyons, M. Sanz-Solé: Absolute continuity of the law of the solution to the 3-dimensional stochastic wave equation. J. Funct. Anal., 206, 1, 1-32, 2004.

[22] Ll. Quer-Sardanyons, M. Sanz-Solé: A stochastic wave equation in dimension 3: smoothness of the law. Bernoulli 10, 1, 165-186, 2004.

[23] D. Revuz, M. Yor: Continuous martingales and Brownian motion. Third edition. Springer-Verlag, Berlin, 1999.

[24] B. L. Rozovsky: Stochastic evolution equations. Linear theory and applications to non-linear filtering. Kluwer, 1990.

[25] L. Schwartz: Théorie des distributions. Hermann, Paris, 1966.

[26] M. Sanz-Solé, M. Sarrà: Hölder continuity for the stochastic heat equation with spatially correlated noise. In: Stochastic analysis, random fields and applications (R.C. Dalang, M. Dozzi, F. Russo Eds.), pp. 259-268, Progress in Probability 52, Birkhäuser, Basel, 2002.

[27] M. Sanz-Solé: Malliavin Calculus with Applications to Stochastic Partial Differential Equations. Fundamental Sciences. Mathematics. EPFL Press, distributed by CCR Press, 2005.

[28] N. Shimakura: Partial differential operators of elliptic type. Translations of Mathematical Monographs, 99. American Mathematical Society, 1992.

[29] C.D. Sogge: Lectures on Nonlinear Wave Equations. Monographs in Analysis, Vol II. International Press, 1995.

[30] E. M. Stein: Singular Integrals and Differentiability Properties of Functions. Princeton University Press, Princeton, 1970.

[31] F. Treves: Basic Linear Partial Differential Equations. Academic Press, 1975. 
[32] J. B. Walsh: An introduction to stochastic partial differential equations, École d'été de Probabilités de Saint Flour XIV, Lecture Notes in Mathematics, Vol. 1180, Springer Verlag, 1986. 